Spellman et al., 2020

2 Prefrontal deep projection neurons enable cognitive flexibility via persistent feedback monitoring

\title{
12 Summary
}

Cognitive flexibility, the ability to alter one's strategy according to changing stimulus-response-reward

Keywords: prefrontal cortex, cognitive flexibility, calcium imaging, optogenetics, projection neurons, executive functioning, feedback monitoring

\section{Introduction}

Cognitive flexibility, the ability to respond to changing environmental contingencies, is critical for any relationships, is critical for acquiring and updating learned behavior. Attentional set-shifting, a test of cognitive flexibility, depends on the activity of prefrontal cortex (PFC). It remains unclear, however, what specific role PFC neurons play and how they interact to support set-shifting. One widely held view is that prefrontal activity biases sensorimotor responses by mediating attention. Using optogenetics and 2-photon calcium imaging, we demonstrate that, while PFC activity does encode attentional sets, this activity does not bias sensorimotor responses. Rather, PFC activity enables set-shifting by encoding trial feedback information, a role it has been known to play in other contexts. We identify a circuit-level mechanism that supports feedback monitoring through persistent, recurring activity bridging multiple trials. Unexpectedly, the functional properties of PFC cells did not vary with their efferent projection targets in this context. Instead, representations of trial feedback formed a topological gradient, with cells more strongly selective for feedback information located further from the pial surface and receiving denser afferent inputs from the anterior cingulate cortex. Together, these findings identify a critical role for deep PFC projection neurons in enabling set-shifting through behavioral feedback monitoring. organism that must navigate through a dynamic environment. A form of cognitive flexibility frequently employed in clinical assessments is attentional set-shifting, a kind of task-switching behavior that requires a subject to ignore a previously relevant stimulus feature and instead attend to a previously irrelevant feature (Heisler et al., 2015; Tait et al., 2014).

Set-shifting impairments are common in a range of psychiatric disorders (Ceaser et al., 2008; Disner et al., 2011; Halleland et al., 2012; Jazbec et al., 2007; Murphy et al., 2012) and often persist after treatment in both depression and schizophrenia, despite remission of other symptoms (Bortolato et al., 2016; Gonda et al., 2015; Harvey et al., 2004). A long-standing body of evidence indicates that the prefrontal cortex (PFC) plays a critical role in supporting set-shifting behavior in human (Kim et al., 2012; Milner, 1963) and rodent models (Birrell and Brown, 2000; Bissonette et al., 2013; Brigman et al., 2005), but the circuit elements and physiological mechanisms that enable set-shifting remain poorly resolved. A functional dissection of the prefrontal circuitry underlying set-shifting is of particular translational interest because pharmacotherapeutic interventions in this circuitry can have complex or paradoxical effects by acting on competing circuit elements (Miller, 2016), which may account for the deleterious cognitive side effects that can accompany antipsychotics and SSRls (Hill et al., 2010; Sayyah et al., 2016). 
Spellman et al., 2020

The dominant model for the role of PFC in set-shifting, which has drawn on support from numerous

49 high-impact publications over the past three decades (Miller and Cohen, 2001; MacDonald et al., 2000;

50 Corbetta and Shulman, 2002; Desimone and Duncan, 1995; Birrell and Brown, 2000; Schmitt et al., 2017),

51 holds that the PFC supports set-shifting by encoding abstract task rules and attentional sets that mediate

52 top-down control of sensorimotor processing and decision making. There is physiological support for this

53 model from animal studies. Neural activity in primate prefrontal cortex encodes abstract contextual or rule-

54 related information (Hymana et al., 2012; Mante et al., 2013; Rigotti et al., 2013), and in rodents performing

55 set-shifting tasks, such rule-related representations can shift flexibly with repeatedly changing stimulus-

56 reward contingencies (Durstewitz et al., 2010; Mante et al., 2013; Rich and Shapiro, 2009; Rodgers and

57 DeWeese, 2014; Siniscalchi et al., 2016). Together, these studies suggest that PFC activity might provide

58 an attentional filter that biases sensorimotor responses during set-shifting (Wimmer et al., 2015) -a well-

59 predicated but as yet unproven hypothesis.

60 Importantly, set-shifting tasks are typically uncued, mirroring the need for uncued adaptations to 61 changing environmental contingencies in the real world, so set-shifting performance requires continuous 62 trial-and-error learning. Thus, an alternative and not mutually exclusive hypothesis is that PFC supports

63 set-shifting by monitoring feedback in response to recent decisions. In addition to encoding context,

64 prefrontal activity has been shown to represent feedback signals associated with trial outcomes (Bissonette

65 and Roesch, 2015; Starkweather et al., 2018), and recent evidence suggests that such feedback-related

66 activity is important for task-switching behavior (Biró et al., 2019; Ellwood et al., 2017). Whether PFC

67 activity supports set-shifting through feedback monitoring or through attentional modulation of sensorimotor

68 responses is unknown.

It is also unclear how distinct circuit elements interact within PFC to support set-shifting. A major set of questions in behavioral neuroscience in recent years has centered on how best to define anatomical markers of cell types within cortex. Are the functional/coding properties of a given pyramidal neuron determined by its efferent connectivity or by its laminar location, a correlate of its afferent connectivity (Adesnik and Naka, 2018; Harris and Shepherd, 2015). These two possibilities are not mutually exclusive, and the fact that neuronal subpopulations with distinct efferent connectivity profiles are often found in distinct cortical layers makes it hard to disambiguate the relative contributions of these two factors. While findings from numerous, recent studies implicate both laminar location and efferent connectivity in determining the functional properties of pyramidal neurons (Lui et al., 2020; Marshel et al., 2019; Senzai et al., 2019; Sharif et al., 2020), to disambiguate these two factors requires an experimental preparation that controls for both. We therefore employed a virallymediated expression approach that allowed for targeted labeling of PFC cell types that differed according to their projection targets or laminar location.

The critical contribution of PFC activity to set-shifting may be mediated by a number of output pathways, but two PFC projection targets of particular interest are the projection to ventromedial striatum (PFC-VMS) and to the medio-dorsal thalamus (PFC-MDT). In rodents, both target structures have established roles in set-shifting behavior (Aoki et al., 2015; Block et al., 2007; Floresco et al., 2006; Kato et al., 2018), and PFC projections to both structures underlie behavior that relies on cognitive flexibility (Marton et al., 2018; Nakayama et al., 2018). However, it is unknown whether PFC projections to these downstream targets convey similar or distinct task-critical information during set-shifting.

To address these questions, we developed and validated a novel set-shifting task for head-fixed mice to enable 2-photon imaging during serial attentional set shifts spanning hundreds of trials. While PFC neural activity encoded all essential task features, neural signals encoding the animal's response were detected only after trial completion, supporting a role for PFC cells in feedback monitoring rather than attentional biasing of sensorimotor responses. This feedback signal was persistent and detectable through four 
bioRxiv preprint doi: https://doi.org/10.1101/828590; this version posted January 19, 2021. The copyright holder for this preprint (which was not certified by peer review) is the author/funder, who has granted bioRxiv a license to display the preprint in perpetuity. It is made available under aCC-BY-NC-ND 4.0 International license.

Spellman et al., 2020

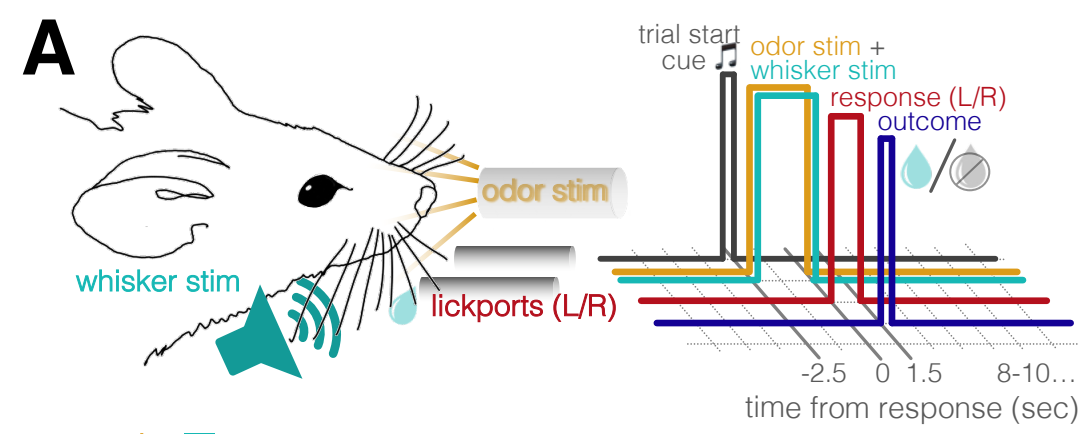

odor whisker

\begin{tabular}{|r|c|c|l|}
\hline training stage & left stim & right stim & irrelevant stim \\
\hline SD & $35 \mathrm{~Hz}$ & $155 \mathrm{~Hz}$ & \\
\hline $\mathrm{CD}$ & $35 \mathrm{~Hz}$ & $155 \mathrm{~Hz}$ & (almond / olive) \\
\hline IDS & $210 \mathrm{~Hz}$ & clicks & (almond / olive) \\
\hline Rev & clicks & $210 \mathrm{~Hz}$ & (almond / olive) \\
\hline EDS & almond & olive & (clicks / 210Hz) \\
\hline IDS2 & orange & sesame & (clicks / 210Hz) \\
\hline SEDS & orange I sesame $\leftrightarrow$ clicks / 210Hz \\
\hline
\end{tabular}

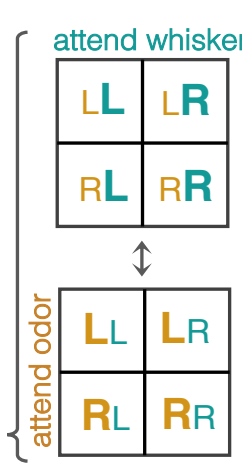

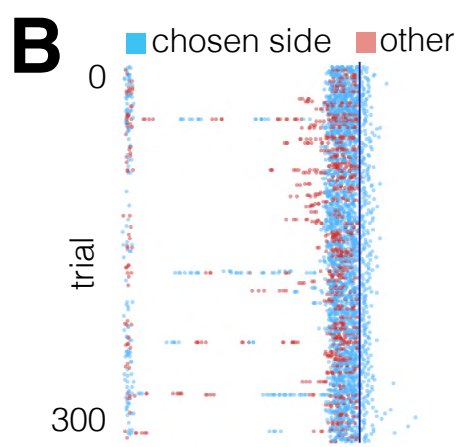

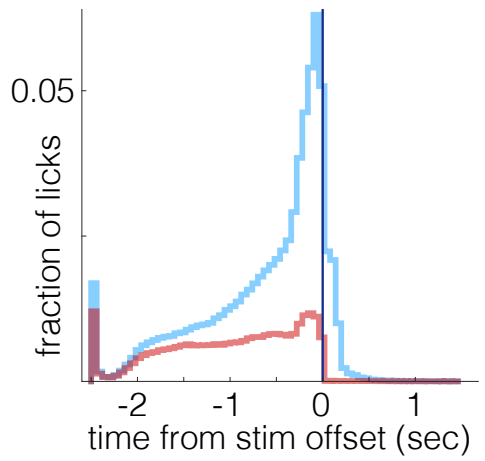

D

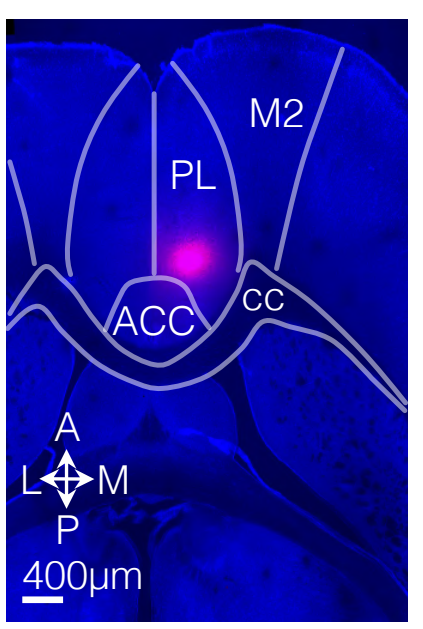

W-rule O-rule

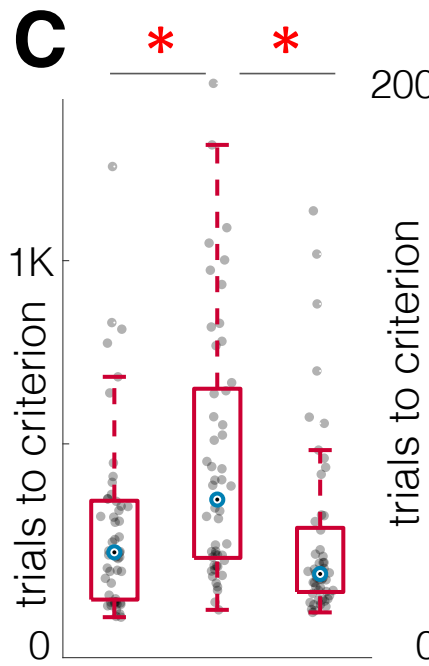

IDS1 EDS IDS2

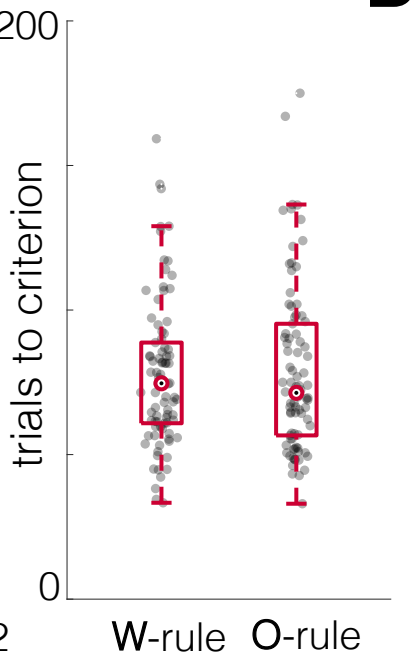

E EDS $*$

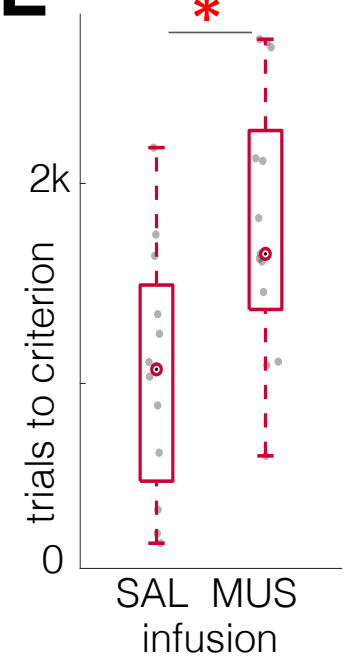

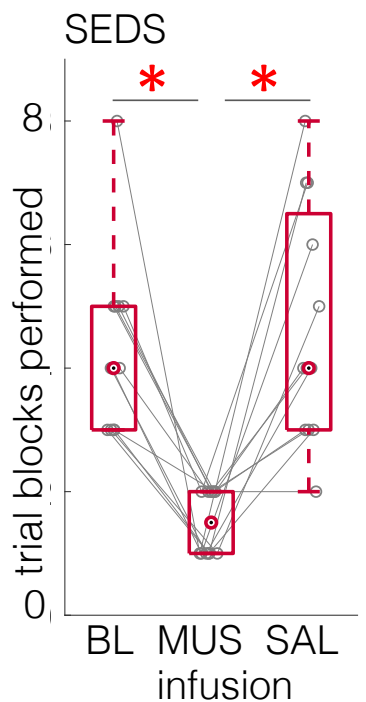

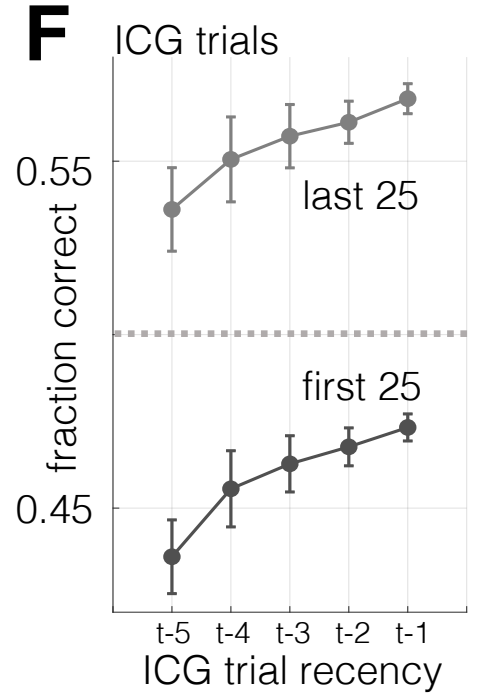

distance from infusion ( $\mu \mathrm{m})$
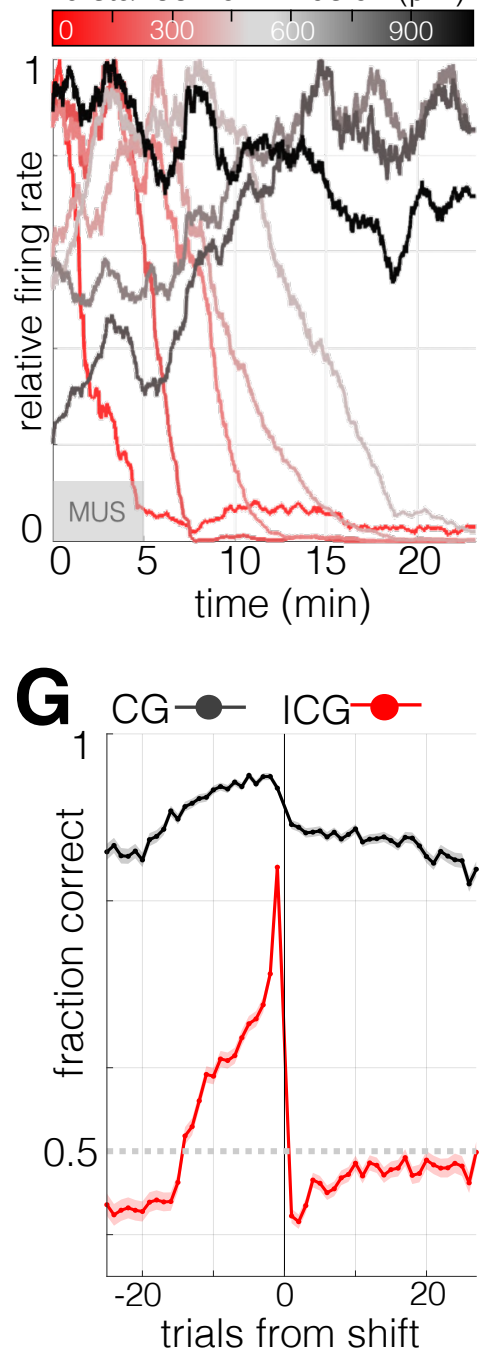


\section{Figure 1: A prefrontal-dependent serial set-shifting task for head-fixed mice}

A. Task schematic. Top left: diagram of stimulus delivery and lick response configuration. Top right: trial sequence. A trial begins with a $500 \mathrm{~ms}$ white-noise trial-start cue, followed by a $2.5 \mathrm{~s}$ presentation of whisker and/or odor stimulus.

Following stimulus offset, there is a response window $(\leq 1.5 \mathrm{~s})$ during which a lick to either lick port terminates the trial, and a correct response is rewarded with a $3 \mu \mathrm{L}$ water bolus. Bottom: a table of the discrete training stages culminating in the serial extra-dimensional set-shifting task. $\underline{\mathrm{SD}}=$ Simple Discrimination (single stimulus modality), $\underline{\mathrm{CD}}=\mathrm{Compound}$ Discrimination (a second, irrelevant stimulus modality is added), $\underline{\mathrm{IDS} 1}=$ Intradimensional Shift (stimuli within the initially relevant modality are replaced with a new set), Rev $=$ Reversal (left/right stimulus/reponse mapping is reversed within initial modality rule), $\underline{\mathrm{EDS}}=$ Extradimensional Shift (same stimuli, new modality rule), $\underline{\mathrm{IDS} 2}=$ Intradimensional Shift (stimuli within the newly relevant modality are replaced with a new set), $\underline{\text { SEDS }}=$ Serial Extradimensional Shifting (the modality rule is repeatedly switched).

B. Summary of licking behavior. Top: rasterized and trial-aligned lick times for an example session. Licks toward the ultimately chosen lick port in blue, licks toward the other lick port in red. On many trials, both lick ports are explored prior to the response window. Bottom: Summary lick time histogram (655,236 licks, 150 sessions, 32 animals). On 95\% of trials, the choice lick (first lick made after stimulus termination) came in the first $0.346 \mathrm{~ms}$ of the response window, a latency equal to a single frame of imaging.

C. Left: Number of trials to criterion, Intradimensional vs Extradimensional Shift, $\mathrm{n}=53$ animals. Signed rank $\mathrm{z}=-2.9, \mathrm{p}=$ 0.0034 , for IDS1/EDS; $\mathrm{z}=-4.0, \mathrm{p}=6 \times 10^{-5}$ for IDS2/EDS, $\mathrm{z}=1.4, \mathrm{p}=0.15$ for IDS1/IDS2. Right: Mean trials to criterion during SEDS sessions, whisker rule vs odor rule. $\mathrm{N}=115$ animals, signed rank $\mathrm{z}=0.14, \mathrm{p}=0.9$.

D. Muscimol infusion in PFC. Left: An infusion of fluorescent muscimol made using same concentration, volume, and infusion rate as in the muscimol behavioral experiment. Right: Relative multiunit firing rate (proportion of channel maximum, $0.5 \mathrm{~Hz}$ bins, $100 \mathrm{~s}$ moving average). Each trace is the mean activity of a pair of electrodes at a given $\mathrm{A} / \mathrm{P}$ distance from the infusion site.

E.Left: trials to criterion in EDS sessions during transcranial infusion ( $\mathrm{SAL}=$ saline; $\mathrm{MUS}=$ muscimol). $\mathrm{N}=12,13$ mice (SAL, MUS). Rank sum $\mathrm{z}=2.4, \mathrm{p}=0.02$. Right: Number of trial blocks reaching criterion performance in SEDS sessions following 10 rule shifts. $N=12$; median blocks (BL/MUS/SAL): 4, 1.5, 4. Signed rank $p=0.0005$ (BL/MUS), 0.001 (SAL/MUS), 0.68 (BL/SAL). Median total trials completed: $\mathrm{SAL}=644$; MUS $=651$; Signed rank $\mathrm{p}=0.52$.

F. Incongruent trial performance by recency of previous incongruent trial during SEDS sessions (mean \pm SEM). Top: performance during the last 25 trials of trial blocks, prior to reaching criterion; bottom: performance during the first 25 trials of trial blocks, after undergoing rule shifts. $\mathrm{N}=693$ sessions in 131 animals. ANOVA for ICG trial recency vs ICG trial performance: $\mathrm{t}=-4.68, \mathrm{p}=3 \times 10^{-6}$.

G. SEDS trial performance relative to rule shift, mean \pm SEM. Same sessions as in $\mathbf{F}$.

98 subsequent trials, spanning up to 55 seconds. Separate analysis of PFC-VMS and PFC-MDT neurons revealed strikingly similar representations of all task-related features in both cell types. Unexpectedly, whereas optogenetic inhibition of either cell type had no effect on performance when delivered during trials, inhibition during the post-trial epoch did impair performance-but only following rule-dependent trials, confirming that the role of these neurons in set-shifting was feedback monitoring and not attentional modulation of sensorimotor responses. In contrast with PFC, inhibition of posterior parietal cortex (PPC) during trials did impair performance, showing that PPC but not PFC is required for attentional modulation of sensorimotor responses. Furthermore, while the functional properties of PFC cells did not vary with their efferent projection targets in this context, we found that representations of trial feedback formed a topological gradient, with cells more strongly selective for feedback information located further from the pial 108 surface and receiving denser afferent inputs from the caudo-ventral anterior cingulate cortex (ACC). 109 Together, these findings reveal a critical role for deep PFC projection neurons in supporting set-shifting by 110 relaying feedback information to downstream targets.

\section{Results}

\section{A cross-modal set-shifting task for head-fixed mice}

116 To image PFC activity during set-shifting behavior in mice, we developed a head-fixed paradigm 117 leveraging the sensitivity of the olfactory and whisker somatosensory systems and permitting us to record 118 from hundreds of cells during serial attentional set shifts spanning hundreds of trials. Water-restricted mice 119 were presented on each trial with one of two possible whisker vibration stimuli (e.g. $35 \mathrm{~Hz}$ vs $155 \mathrm{~Hz}$ vibration 
Spellman et al., 2020

120 presented bilaterally) and one of two possible odor stimuli (e.g. almond oil vs olive oil), in randomized

121 combinations, cueing them to respond by licking a left or right lick ports to retrieve a water reward upon

122 termination of the 2.5s compound stimulus (Fig. 1A-B). As in most set-shifting tasks (Bissonette et al.,

123 2008; Tait et al., 2014), animals underwent a standardized series of task transitions in order to expose

124 them to multiple exemplars from each stimulus modality and build an attentional set: simple discrimination

125 (SD), in which animals were trained to discriminate between two stimuli within a single sensory modality;

126 compound discrimination (CD), in which a distractor stimulus from the untrained sensory modality was

127 added; intradimensional shift (IDS), in which the stimuli from the relevant sensory modality were replaced

128 with a new pair of exemplars; reversal (Rev), in which the left/right mapping was switched; extradimensional

129 shift (EDS), in which the rule changed for the first time so that the pair of stimuli from the previously

130 irrelevant sensory modality became the relevant stimuli; a second intradimensional shift (IDS2), in which

131 the pair of stimuli from the newly relevant sensory modality was replaced with a new pair of exemplars; and

132 finally serial extradimensional shifts (SEDS), in which the rule switched automatically whenever the animal

133 reached criterion performance, which was $80 \%$ correct within a 30 -trial moving window, and $>50 \%$ on both

134 left and right trials (Fig. 1A, see Methods for details). To validate the set-shifting task, we confirmed that

135 animals took longer to perform an EDS than an IDS, and there was no difference between modalities in

136 trials to criterion (Fig. 1C). Infusion of muscimol within PFC (Fig. 1D) impaired set-shifting performance,

137 increasing trials to criterion in both the initial EDS shift, as well as after completing 10 shifts of SEDS (Fig.

138 1E), showing that set-shifting in this paradigm is dependent on PFC activity, both initially and after repeated

139 shifts.

140 Trials belonged to two classes: those in which the whisker and odor rules cued the same response 141 direction (congruent trials: CG), and those in which the whisker and odor rules cued opposing response

142 directions (incongruent trials: ICG). Therefore, ICG trials, but not CG trials, required application of the

143 modality rule, and feedback from ICG trials alone carried information about modality rule. Animals used

144 prior ICG trial information to guide behavior, so that ICG trial performance was enhanced following

145 feedback from a recent ICG trial, and this held true in trials occurring both early and late within trial blocks

146 (Fig. 1F). Performance on ICG trials, but not CG trials, dropped below chance immediately following rule

147 switches, revealing a perseverance of the previous rule; sub-chance performance persisted over multiple

148 ICG trials, gradually reaching chance and then above-chance performance as new the new rule was

149 acquired (Fig. 1G).

\section{Set-shifting task variables represented in prefrontal population activity}

151 To measure task-related activity in PFC neurons, GCaMP6f-mediated 2-photon calcium imaging was

152 performed through a coronally-implanted microprism (Andermann et al., 2013; Low et al., 2014), producing

153 a field of view that preserved cortical laminar structure and was restricted to prelimbic and infralimbic areas

154 (Fig. 2A-B). This field of view allowed for monitoring of a $1500 \mu \mathrm{m} \times 760 \mu \mathrm{m}$ area, with a median of 450

155 active neurons per animal in a given session (registering cells across consecutive sessions yielded a

156 median of 179 per animal - see Methods and Fig. S3-S10). GCaMP was expressed pan-neuronally (hSyn-

157 GCaMP6f) or in specific projection neuron subtypes (hSyn-DIO-GCaMP6f and rAAV2-Cre in PFC-VMS

158 and PFC-MDT). Although these cell types were subsequently analyzed separately, we began by

159 characterizing the task-responsiveness of all labeled neurons. Examination of activity traces averaged over

160 feature-matched trials revealed neurons whose response profiles displayed compound selectivity for

161 multiple task features, such as whisker stimulus and odor stimulus (Fig. 2C, left).

162 To ascertain which task-related features were encoded at the population level, we began by using

163 tensor component analysis (TCA, Fig. 2D), to decompose the neural data into low-rank factors defined as

164 related sets of weights in the neuron, trial, and timepoint (within-trial) dimensions (Williams et al., 2018).

165 These weights reflect the magnitude of the underlying activity pattern at each neuron, trial, and timepoint.

166 Because this dimensionality reduction technique separates trial and timepoint components, it allowed for

167 variables to be directly compared with each other, regardless of when their peak representation occurred

168 within the trial. Using the trial components as inputs to a support vector machine-based (SVM) maximum-

169 margin linear decoder (Christianini and Shawe-Taylor, 2000; Meyers et al., 2008), trials were classified 
bioRxiv preprint doi: https://doi.org/10.1101/828590; this version posted January 19, 2021. The copyright holder for this preprint (which was not certified by peer review) is the author/funder, who has granted bioRxiv a license to display the preprint in perpetuity. It is made available under aCC-BY-NC-ND 4.0 International license.

Spellman et al., 2020

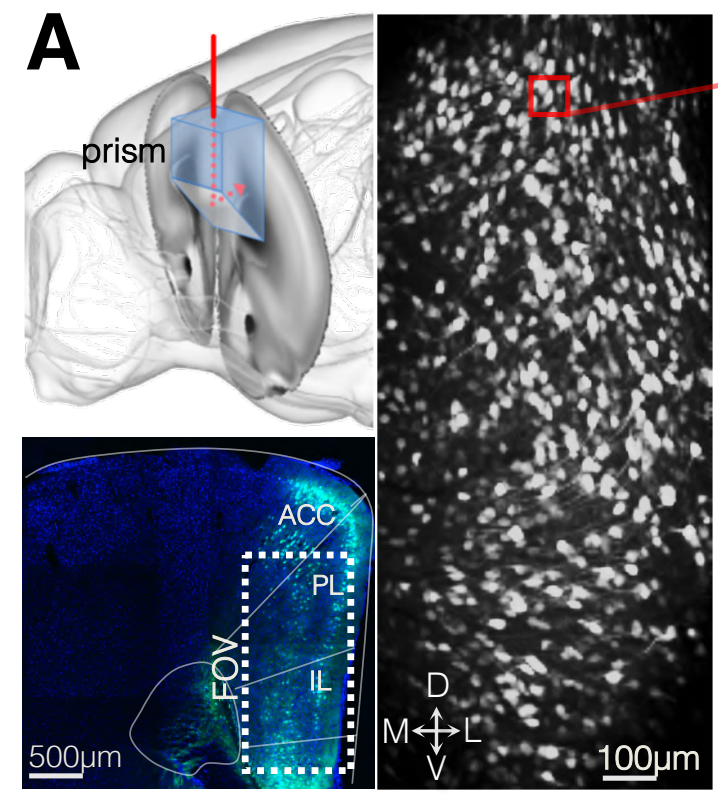

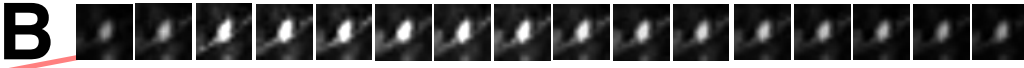
raw signal

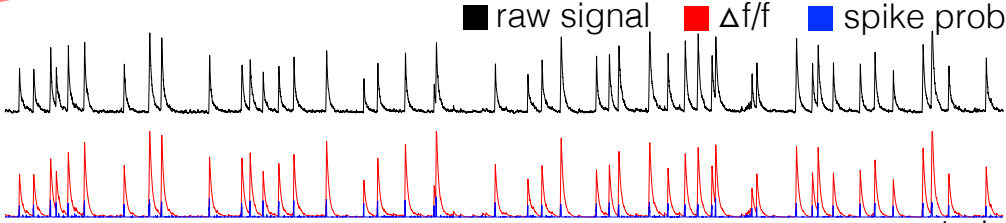

whisker rule odor rule

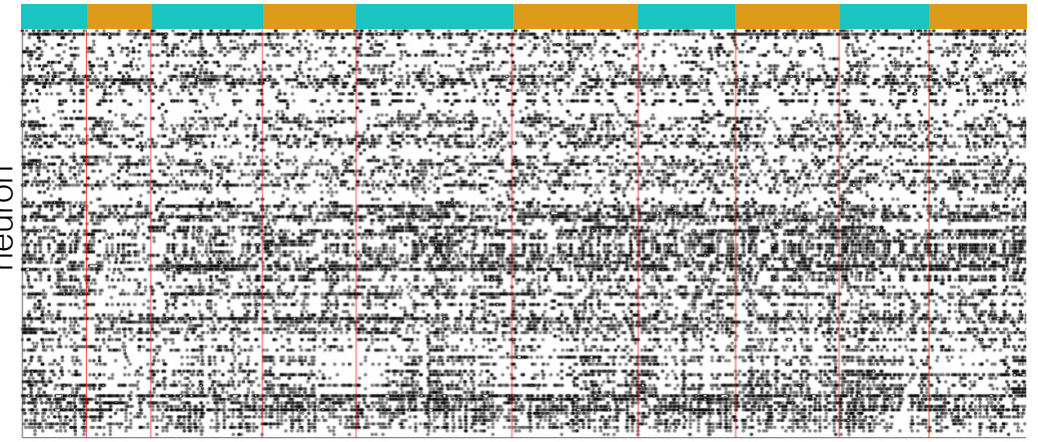

0

time (min)

80
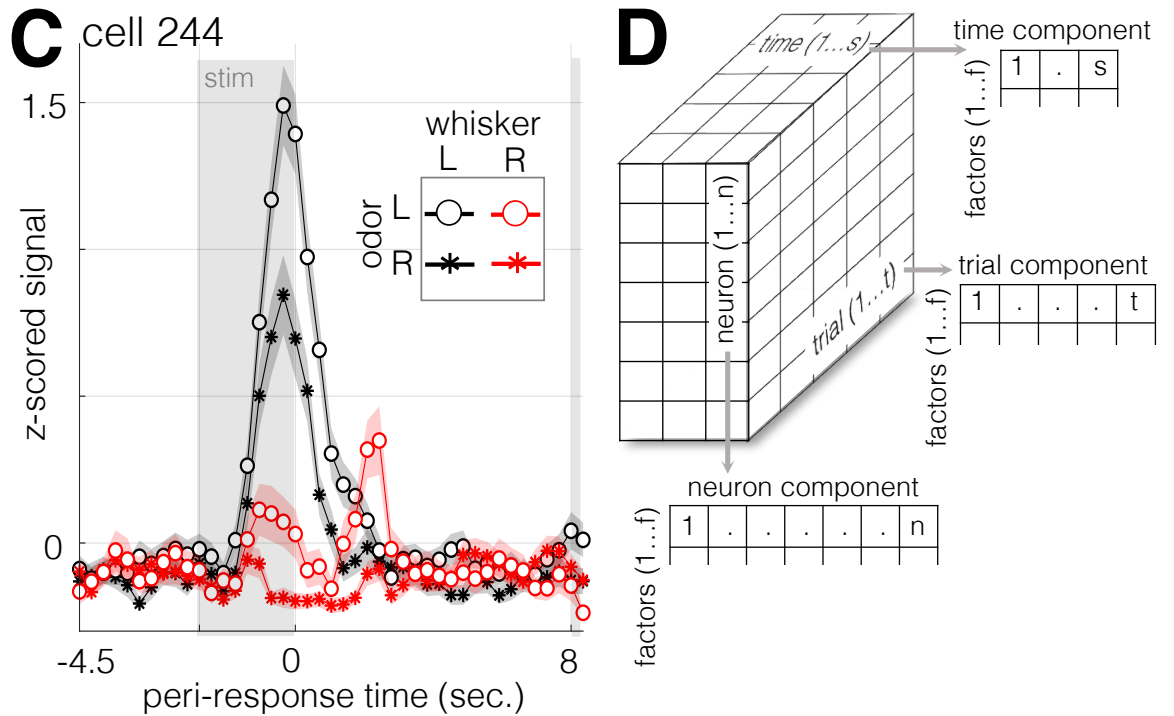

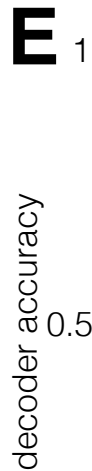
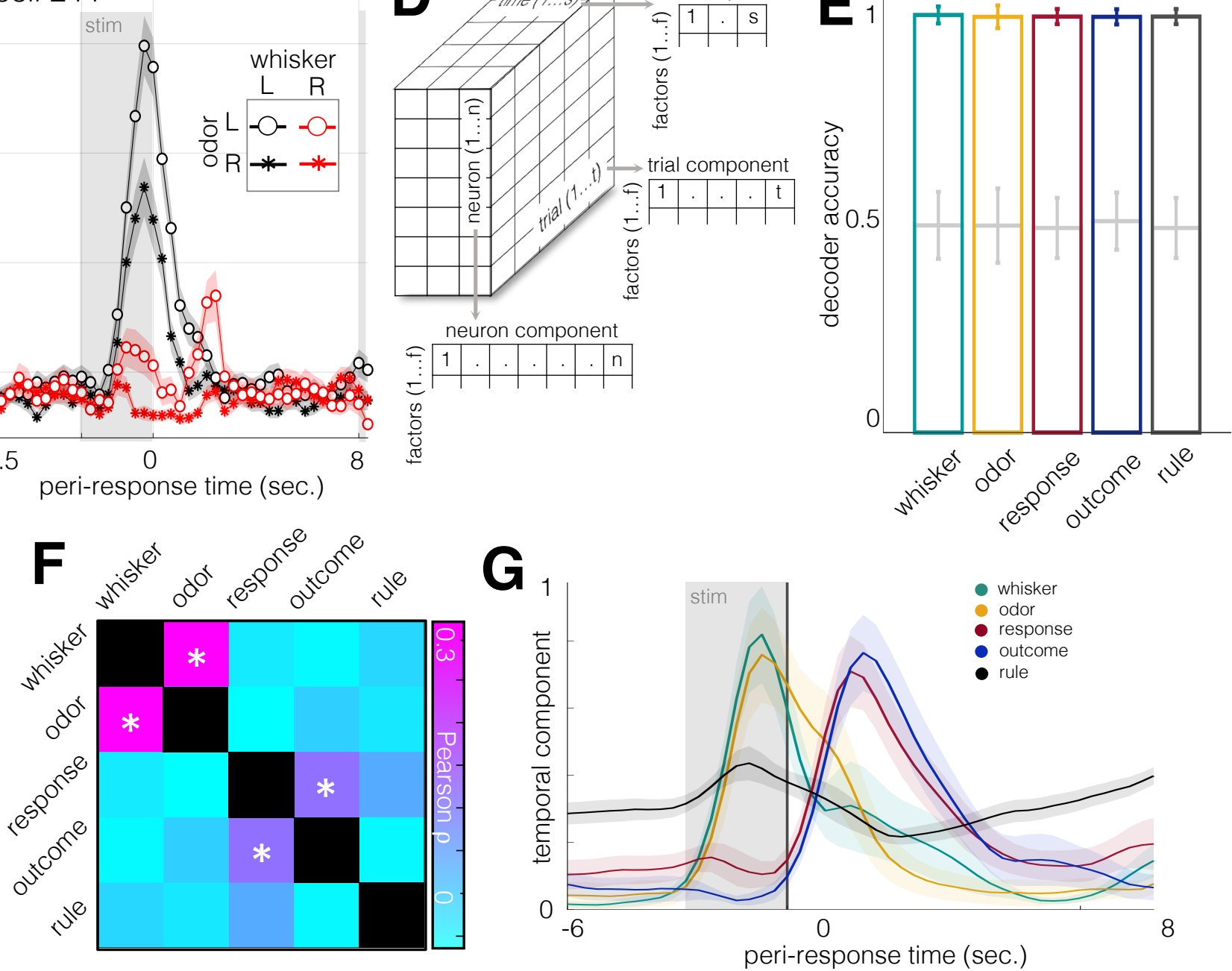
Figure 2 Prefrontal representation of task variables in population activity

A. Top left: schematic of coronal prism implantation field. FOV bregma coordinates: AP $+1.85 \mathrm{~mm}$, ML $0-0.75 \mathrm{~mm}$ left, DV

$1.0 \mathrm{~mm}-2.5 \mathrm{~mm}$ from brain surface. Bottom left: hSyn-GCaMP6f fluorescence in fixed tissue (DAPI in blue). Right: standard deviation image from 115 -minute hSyn-GCaMP6f recording $(2.89 \mathrm{~Hz}, \mathrm{~N}=953$ units). Note: different animal from bottom left.

B. Example frames, traces, and putative spike times from calcium imaging sessions. Top: 16 frames of a calcium transient from an example neuron in (A). Frames are downsampled at a ratio of 1:2, so that the sequence shown is at $1.45 \mathrm{~Hz}$. Black trace: raw image fluorescence. Red trace: denoised $\Delta \mathrm{f} / \mathrm{f}$. Blue: putative spike times from deconvolved $\Delta \mathrm{f} / \mathrm{f}$ signal. Bottom: rasterized putative spike times from SEDS session. $\mathrm{N}=100$ units recorded over the course of 10 trial blocks.

C. Mean \pm SEM of z-scored activity for all trials from an example neuron during trials with four stimulus combinations (whisker stimulus right/left, odor stimulus right/left). Stimulus is presented from $-2.5 \mathrm{~s}$ to $0 \mathrm{~s}$. Earliest subsequent trial onset at $8 \mathrm{sec}$.

D. Schematic of TCA rank decomposition. A three-dimensional data set (time of length s, neurons of length $\mathrm{n}$, and trials of length $t$ ) is decomposed into three lower-rank components of $f$ factors $(s * f, n * f$, and $t * f)$.

E. SVM decoder accuracy for five task-related features. Trial factors obtained from TCA decomposition ( 35 factors) were used as inputs to a support vector machine-based decoder, which was trained to classify trials according to five features: whisker stimulus, odor stimulus, response direction (left / right), trial outcome (correct / incorrect), and task rule (whisker rule / odor rule). Cross-validation was performed on trials (50\%) held out from training. $\mathrm{N}=4740$ neurons from 21 animals. Error bars are standard deviations. Gray lines and error bars are performance on shuffled labels.

F. Cross-correlation of task feature representation in TCA trial space. GLM regression was performed on the five task variables, using TCA factors as predictors. Pearson correlation was then performed on the resulting coefficients (same data as in E). Asterisks indicate $\mathrm{p}<0.01$.

G. Time components from significantly modulated factors. For each of the five task variables, factors most strongly modulated by each task variable are shown in the temporal domain (mean + - SEM for each variable, $\mathrm{N}=16$ factors for whisker stimulus, $\mathrm{N}=12$ factors for odor stimulus, $\mathrm{N}=26$ factors for response direction, $\mathrm{N}=21$ factors for trial outcome, $\mathrm{N}=59$ factors for rule).

according to whisker stimulus (e.g. clicks vs. $210 \mathrm{~Hz}$ ), odor stimulus (e.g. almond vs. olive), response (left 174 / right), outcome (correct / incorrect), and rule (whisker rule / odor rule) with near perfect accuracy in held175 out test data (Fig. 2E).

We next sought to determine the degree to which representations of these task variables correlated with one another within the population activity space. To determine whether each task variable was encoded by a distinct pattern of activity from across the neuron population, or alternatively, whether multiple task variables were encoded by similar activity patterns, we performed general linear model (GLM)

ression on the trial components, using the five variables as predictors, and then tested for correlations

181 between the resulting coefficients across factors (Fig. 2F). Coefficients for whisker and odor stimuli were

182 significantly correlated with each other across factors, as were response and outcome, indicating these

183 pairs of variables were encoded by similar activity patterns. One possible way this could manifest is if

184 activity patterns encoding whisker and odor follow overlapping temporal trajectories within the trial, and

185 likewise for response and outcome. To determine the trial-aligned time-courses of the factors associated

186 with the task variables, we plotted the TCA-derived temporal components of the factors most strongly

187 associated with each variable (Fig. 2G). Temporal components associated with whisker and odor stimulus

188 peaked during stimulus presentation, while components associated with outcome peaked during the

189 subsequent inter-trial interval (ITI). Notably, components associated with response followed a temporal

190 trajectory that was more similar to outcome than whisker or odor stimuli, peaking after the end of the trial

191 (Fig. 2G). These results demonstrate that all task elements necessary for successfully executing the task

192 are represented in PFC neuronal population activity, that activity patterns associated with response are

193 more correlated with those associated with outcome than with those associated with the decision-related

194 stimuli, and that the response-associated patterns lag, rather than lead, the animal's behavioral choice.

196 Response and outcome representations in post-trial activity

197 Next, we questioned how the activity of individual neurons contributed to the representation of task

198 variables seen in the population-level analysis. Because the TCA-based population analysis revealed

199 signals encoding response and outcome in the post-trial ITI, we wondered whether these variables might 
bioRxiv preprint doi: https://doi.org/10.1101/828590; this version posted January 19, 2021. The copyright holder for this preprint (which was not certified by peer review) is the author/funder, who has granted bioRxiv a license to display the preprint in perpetuity. It is made available under aCC-BY-NC-ND 4.0 International license.

Spellman et al., 2020
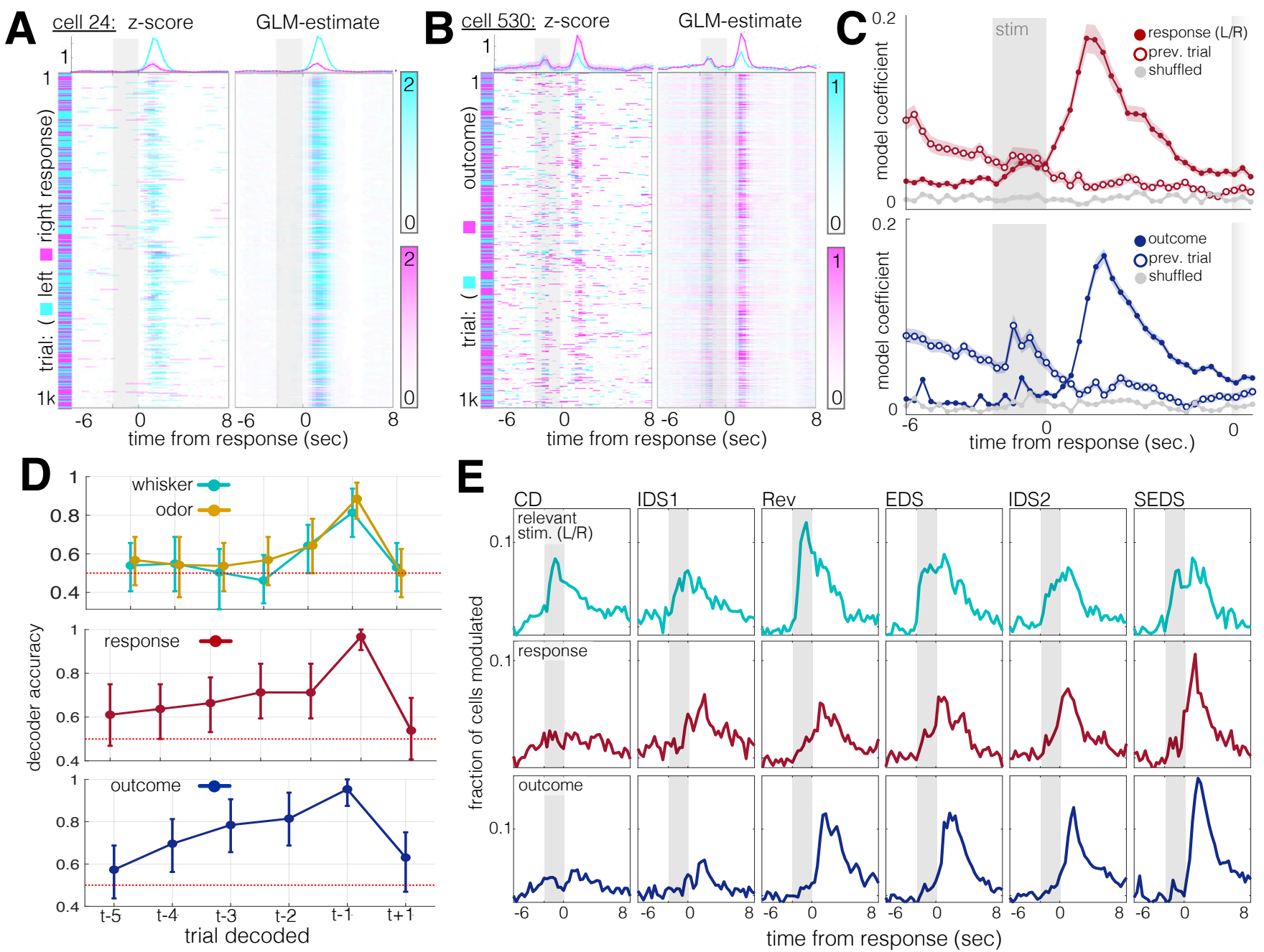

time from response (sec)

Figure 3: Task-related action and outcome representations in post-trial activity

A. Example neuron with selectivity for response direction in its post-trial activity. Left: z-scored activity. X-axis, time within trial (Gray box: stimulus presentation window, $-2.5 \mathrm{~s}-0 \mathrm{~s}$ ). Y-axis: trial number (1071 trials total). Right: GLM estimate for time-aligned activity. Cyan: trials in which the animal chose left; magenta: trials in which the animal chose right. Top: mean +/- SEM traces from each trial condition. Top y-axis units are z-scored activity.

B. Same format as (A), for an example neuron selective for trial outcome. Cyan: incorrect trials; magenta: correct trials.

C. Trial-aligned GLM coefficients for neurons significantly modulated by response direction (top) and trial outcome (bottom). Modulated neurons include those with significant modulation at any timepoint (Bonferroni-corrected for the 43 trial timepoints). Closed circles: mean +/- SEM of coefficients for currently trial; open circles: mean +/- SEM of coefficients for previous trial. Gray traces: coefficients from GLMs on shuffled data. N=285 neurons for response direction, 756 for trial outcome, out of 4730 total neurons from 21 animals.

D. Means $\pm 95 \%$ confidence intervals for SVM decoders tested on past $(\mathrm{t}-\ldots)$ and future $(\mathrm{t}+\ldots)$ trial features. Top: decoders for whisker and odor stimulus. Middle: decoders for response direction. Bottom: decoders for trial outcome. Red dashes: chance performance.

E. Histograms: fraction of neurons significantly modulated (same GLM as A-C) by task features over trial-aligned timepoints and through learning stages. Top row: fraction of neurons modulated by the relevant stimulus. Middle row: response direction. Bottom row: trial outcome.

continue to be represented in the neural signal beyond the start of the subsequent trial. To answer this question, we used GLM regression to model activity rates in individual neurons as a function of whisker stimulus, odor stimulus, response direction trial outcome, and rule, with the addition of the variables previous trial response and previous trial outcome. Fig. 3A-B shows example trial-aligned activity traces, along with GLM estimates for trial-by-trial activity, for two example neurons encoding response and 
206 outcome in post-trial activity (see Fig. S12 for further examples). Analysis of the mean GLM coefficients at 207 each timepoint (Fig. 3C) revealed that coefficient values for response and outcome peaked at a mean of 208 1.73s and 2.42s after stimulus offset, respectively, and remained elevated well above values from shuffled 209 data through the following trial.

210 The observation that representations of response and outcome persisted through the subsequent trial 211 raised the possibility that these signals might be detected beyond this time interval. Given that animals

212 required 15-20 incongruent trials to abandon a rule after an uncued rule-change (Fig. 1E), we looked for

213 evidence of outcome-related evidence accumulation that spanned multiple trials, as has been reported in

214 reinforcement learning tasks (Bari et al., 2019; Bernacchia et al., 2011; Siniscalchi et al., 2019). We ran

215 linear decoders on whisker and odor stimuli, response, and outcome for past trials and found that, while

216 whisker and odor representations were no longer detectable after one trial, response and outcome

217 representations persisted for up to four trials, or up to 55 seconds (Fig. 3D). Importantly, the inability to

218 decode these features at above-chance accuracies from future trials (timepoint $t+1$ in Fig 3D) served as a

219 negative control against potential autocorrelation effects in behavior and/or neural activity.

220 Do these response and outcome signals reflect the demands of the uncued set-shifting task, or are they

221 natively expressed during decision-making more generally? To answer this question, we tracked response

222 and outcome signals across learning stages. Signals corresponding with the relevant stimulus was present

223 from the earliest recorded session (CD) and remained strong throughout all subsequent task stages, (Fig.

224 3E, top). Conversely, very few neurons were modulated by response or outcome in early (e.g. CD)

225 sessions. Instead, response- and outcome-related activity emerged only over the course of multiple task

226 transitions (Fig. 3E, rows 2 and 3). While this analysis does not precisely identify the minimum conditions

227 necessary to evoke the response and outcome signals, the absence of these signals in early sessions

228 means that these signals do not innately result from discrimination behavior, but require extensive training

229 and/or task complexity.

231 A circuit-level mechanism representing outcomes across multiple trials in a stable, colinear activity

\section{2 space}

233 That trial feedback information could be decoded for up to four trials in the past led us to question how

234 these past trial outcomes were encoded: were these representations stable, maintained by consistent groups of neurons, or by groups whose membership shifted over time? We first examined the stability of outcome selectivity across sessions. Outcome selectivity, defined hereafter as the difference between mean z-scored activity in correct and incorrect trials for each neuron, exhibited low correlation between pairs of early sessions, when the representations themselves were weak (Fig. 4A). Between later session pairs, however, selectivity became highly correlated, especially among SEDS sessions (Fig. 4B-C), indicating that outcome is represented by relatively stable groups of neurons over days.

We then examined the temporal stability of outcome signals over trials. The neurons responsible for coding a given trial outcome might shift between trials, with different groups of neurons inheriting representations of different past trials. Alternatively, these trial histories might be multiplexed by the same neurons, whose activity could be incrementally modulated with each correct and incorrect trial. To test these possibilities, we compared outcome selectivities for successive past trials and found that they were indeed supported by correlated populations, whereby, e.g., neurons excited by reward in trial t- 1 were also excited by reward in trial t-2 (Fig. 4D). As a further illustration of this phenomenon, the path of each neuron's incorrect/correct ratio over successive past trials tended to display a linear regression toward unity (Fig. 4E).

250 To better understand the evolution of outcome representations over trials, we analyzed the relative 251 locations of these representations in the activity space. The representation of each recent trial might be 252 encoded separately and relayed to downstream brain regions for further processing, or alternatively, they 253 might be compressed into a low-dimensional readout, signaling the animal's overall performance. An 254 analysis of the Euclidean distances and vector angles of pairs of past outcomes in the neural activity space 255 (Fig. 4F) revealed that more similar outcome combinations (e.g. [t-1 incorrect, t-2 correct] vs [t-1 correct, t2562 incorrect]) were less distant than more different outcome combinations (e.g. [t-1 incorrect, t-2 incorrect] 257 vs [t-1 correct, $t-2$ correct]), and that all pairs of outcome vectors were more colinear (exhibiting lower vector 
bioRxiv preprint doi: https://doi.org/10.1101/828590; this version posted January 19, 2021. The copyright holder for this preprint (which was not certified by peer review) is the author/funder, who has granted bioRxiv a license to display the preprint in perpetuity. It is made available under aCC-BY-NC-ND 4.0 International license.

Spellman et al., 2020

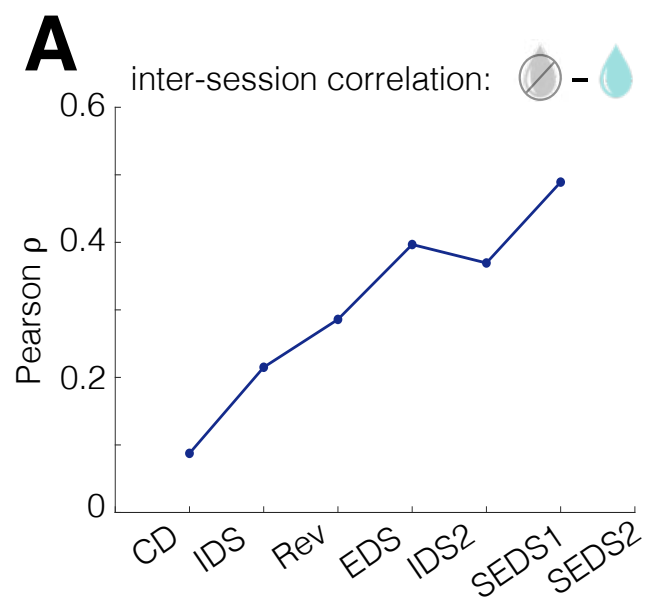

D

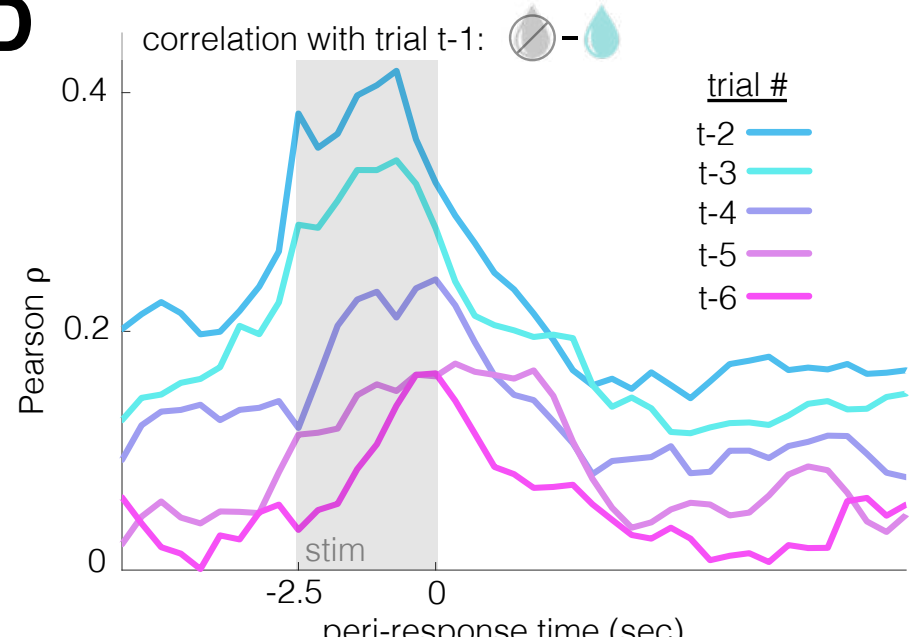

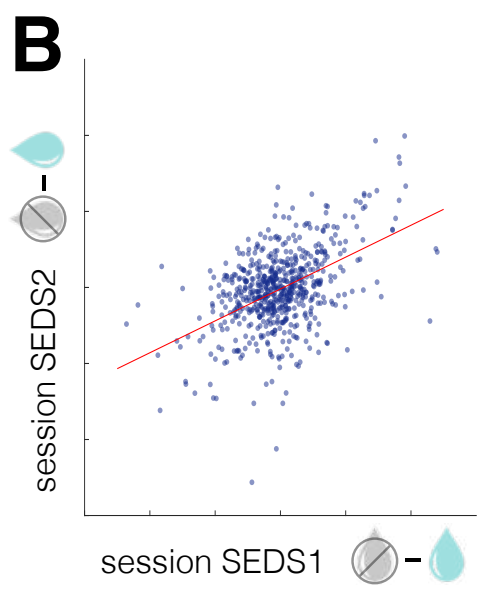
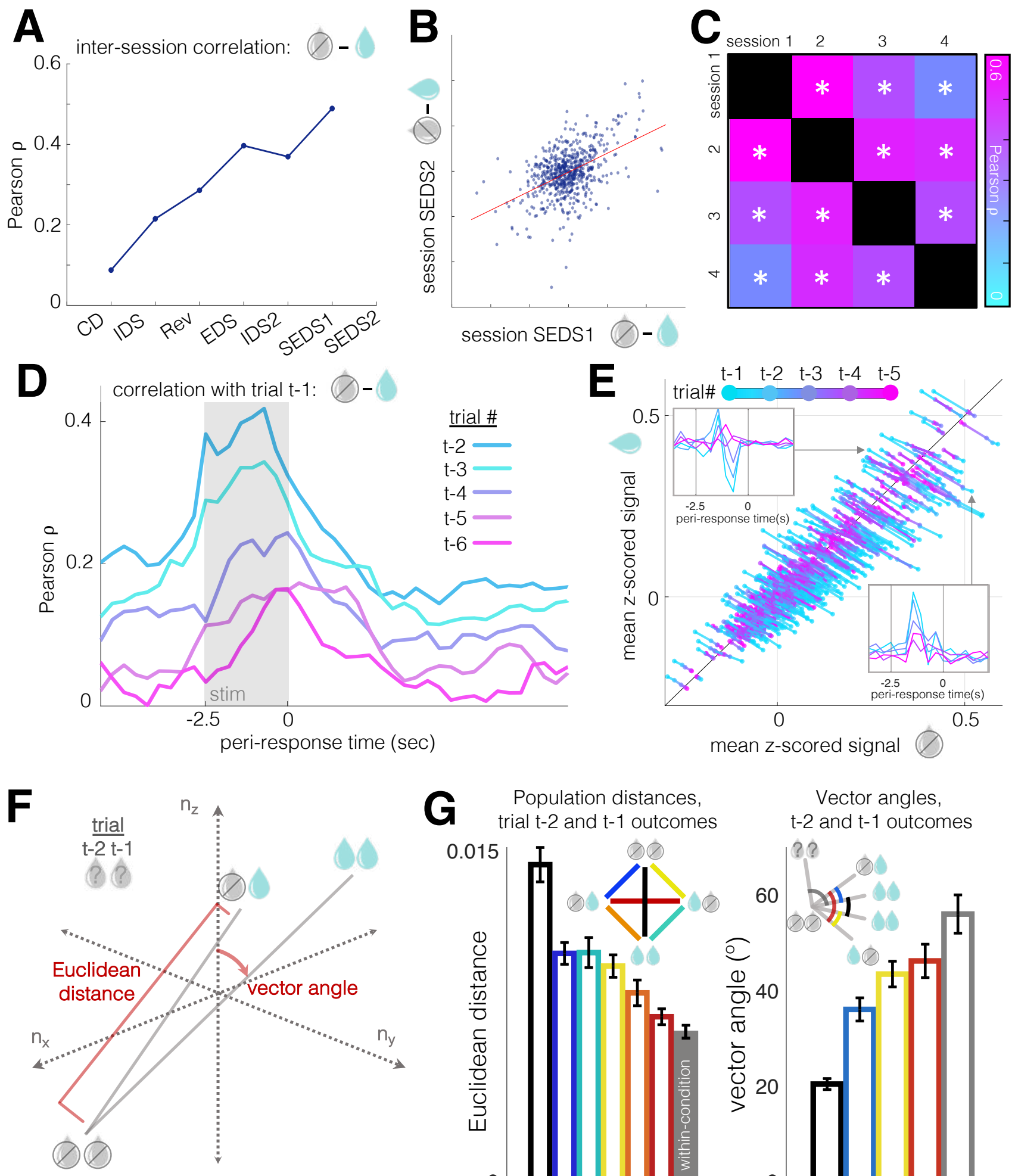

G
Population distances, trial t-2 and t-1 outcomes

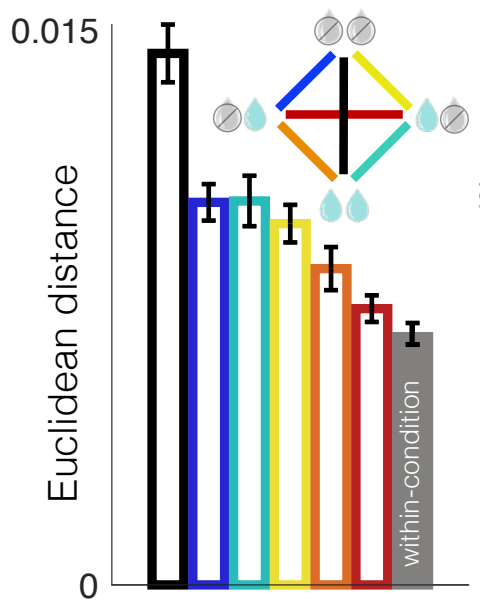

Vector angles, $\mathrm{t}-2$ and $\mathrm{t}-1$ outcomes

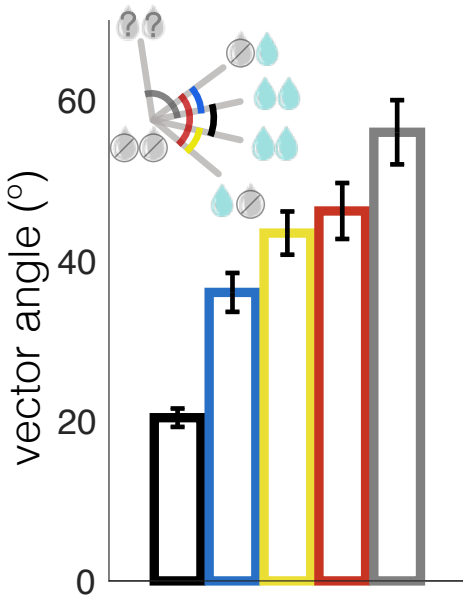


bioRxiv preprint doi: https://doi.org/10.1101/828590; this version posted January 19,2021 . The copyright holder for this preprint (which was not certified by peer review) is the author/funder, who has granted bioRxiv a license to display the preprint in perpetuity. It is made available under aCC-BY-NC-ND 4.0 International license.

Spellman et al., 2020

Figure 4: Outcomes are represented across multiple trials in a stable, colinear activity space

A. Inter-session correlation of trial outcome representation. X-axis: imaging sessions in which each significantly modulated neuron's outcome selectivity (tuning) was tracked across pairs of consecutive days. From CD to IDS, $\mathrm{N}=1083$ neurons in 17 animals; from IDS to Rev, 1543 neurons in 16 animals; from Rev to EDS, 1382 neurons in 15 animals; from EDS to IDS2, 1443 neurons in 15 animals; from IDS to the first SEDS session, 1519 neurons in 21 animals; from the first to second SEDS sessions, 635 neurons in 21 animals. Neurons included were those with significant modulation by trial outcome (rank sum, $\mathrm{p}<0.05$ ) in at least one of the two consecutive sessions. Y-axis: Pearson correlation coefficient for outcome selectivity, measured as the difference in mean z-scored activity, averaged over the intertrial interval, between incorrect and correct trials.

B. Scatter plot of outcome selectivities for individual, significantly modulated, neurons in the first and second SEDS sessions (same neurons as A). Pearson $\mathrm{R}=0.49, \mathrm{p}=1.6 \times 10^{\wedge}-39$. Correlation for all neurons (unmodulated as well as modulated) was $0.36, \mathrm{p}=7.5 \times 10^{\wedge}-60 \mathrm{n} \mathrm{N}=1959$ neurons.

C. Similarity of outcome selectivity over multiple SEDS sessions. 173 neurons, from 8 animals, recorded in at least four consecutive behavioral sessions. Neurons significantly modulated by trial outcome in at least one session (rank sum $\mathrm{p}<0.05$ ) were included, though analysis of all neurons produced comparable results ( 1091 neurons, $\mathrm{R}=0.17-0.38$, all $\mathrm{p}$ values $<1 \times 10^{\wedge}-7$ ).

D. Trial-aligned correlation coefficients comparing outcome selectivity (defined as in A-C) between trial t-1 and trials t-2 through t-6. $\mathrm{N}=4740$ cells from 21 animals. Gray box: stimulus presentation.

E. A time-compressed representation of the results from (D), broken out by individual neurons. X-axis: mean activity on incorrect trials; y-axis: mean activity on correct trials. Insets: example cells preferring correct (top) and incorrect (bottom) outcomes in trials t-1 to t-5.

F. Schematic diagram of Euclidean distance and vector angle computed from trial-averaged outcome (correct vs incorrect) conditions in neural activity space.

G. Left: Euclidean distances for all combinations of trial $\mathrm{t}-1$ and $\mathrm{t}-2$ outcomes in N-dimensional activity space (same data as A-E). Bar heights and error bars are means $\pm 95 \%$ confidence intervals over 500 trial sub-samplings with replacement ( $50 \%$ of trials in one condition, $50 \%$ in the other). The rightmost (gray) bar is the mean distance within condition (e.g. [t-1 correct, t-2 correct] vs [t-1 correct, t-2 correct]) across sub-samplings. Right: angles between pairs of trial outcome vectors shown at left. All vectors use [t-1 incorrect, t-2 incorrect] as vertex. Bar heights and error bars are mean $\pm 95 \% \mathrm{CI}$ as at left. Leftmost (black) bar is the mean within-condition angle, a proxy for baseline, as more dimensions tend to increase vector angles in noisy data (Brinkman and Charikar, 2005).

angles) with one another than with vectors from shuffled trials (Fig. 4G). Together, these results define a coding scheme in which a subset of PFC neurons represent the outcome of each trial, with their activity state modulated in an incremental, relatively colinear way, with decreasing amplitude as trials recede into the past, rather than different groups of neurons representing outcomes at different latencies from each trial.

\section{Similar task responsiveness in two major PFC projection neuron populations}

Our findings above indicate that PFC cells are highly functionally heterogeneous with respect to their contributions to cognitive flexibility in this set-shifting paradigm. To understand the mechanistic basis of this functional heterogeneity, we questioned whether the task-related activity of a PFC neuron might be a function of its long-range efferent connectivity profile or of its laminar location, a correlate of afferent connectivity. While not mutually exclusive, these two proposed correlates of functional specialization within cortex have motivated numerous studies in recent years, and noteworthy findings have lent support to both hypotheses. Pioneering work by Otis et al, for example, found dissociated response properties in corticostriatal and cortico-thalamic neurons in a Pavlovian conditioning task, with the former activated and the latter inhibited by reward-predicting conditioned stimuli (Otis et al., 2017). However, because the projection populations examined in that study were located in different cortical layers (layer 2/3-5 and layer 6, respectively), the contribution of laminar depth to coding differences could not be directly addressed. Recent work by Liu et al demonstrated that ventral hippocampal afferents within mouse infralimbic cortex show both projection and layer specificity in their axonal targets, preferentially driving cortico-cortical projection neurons over other projection subtypes, and preferentially driving layer 5 neurons over layer 2/3

280 neurons (Liu and Carter, 2018). Thus, projection specificity and laminar depth may both contribute to 281 functional specialization of subpopulations within PFC. 
bioRxiv preprint doi: https://doi.org/10.1101/828590; this version posted January 19,2021 . The copyright holder for this preprint (which was not certified by peer review) is the author/funder, who has granted bioRxiv a license to display the preprint in perpetuity. It is made available under aCC-BY-NC-ND 4.0 International license.

Spellman et al., 2020
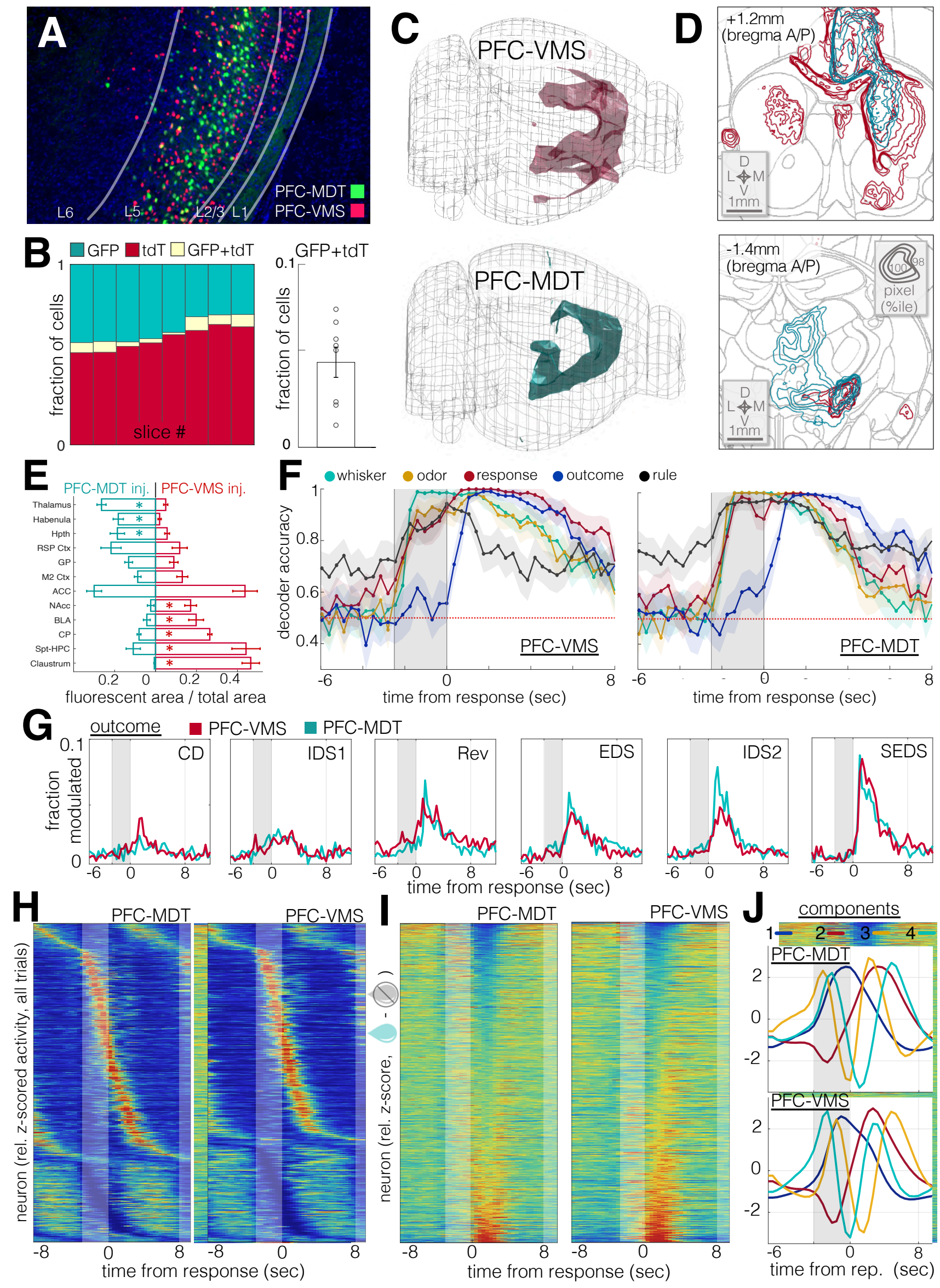
bioRxiv preprint doi: https://doi.org/10.1101/828590; this version posted January 19,2021 . The copyright holder for this preprint (which was not certified by peer review) is the author/funder, who has granted bioRxiv a license to display the preprint in perpetuity. It is made available under aCC-BY-NC-ND 4.0 International license.

Spellman et al., 2020

Figure 5: PFC-VMS and PFC-MDT projection populations exhibit similar task response properties

A. Dual PFC-VMS and PFC-MDT projection labeling in a single preparation. Green: rAAV2-CAG-mNeuroGFP in MDT; red: AAV1-hSyn-DIO-nls-tdTomato in PFC and CAV2-Cre in VMS (DAPI in blue).

B. Cell counts by slice from the experiment in (A). Left: relative green, red, and double-labeled cell counts. Right: median relative overlap $(\mathrm{N}=8$ sections, 2 animals).

C. Composite images of long-range axon projections from PFC-VMS ( $\mathrm{N}=5$ animals) and PFC-MDT ( $=5$ animals) labeling (see Methods). Volumes shown are thresholded to $\geq 98^{\text {th }}$ percentile for pixel brightness.

D. Coronal sections and contour plots of fluorescent axon density in PFC-VMS (top) and PFC-MDT (bottom) labeled animals. Top: a composite ( $\mathrm{N}=5$ animals) of pixel brightness in a coronal cross-section centered on the nucleus accumbens. Concentric contours corresponds with relative pixel brightness (outermost contour, $98^{\text {th }}$ percentile, innermost contour, $100^{\text {th }}$ percentile). Bottom: composite ( $\mathrm{N}=5$ animals) of pixel brightness in a coronal cross-section centered on medial thalamus. Contour brightness scale as in (top).

E. Mean $+/$ - SEM plots showing the fraction of pixels in each region showing fluorescence above the threshold percentile. 2-way ANOVA revealed an interaction effect between injection type and region $(\mathrm{F}=17.96, \mathrm{p}=0)$. Post-hoc $\mathrm{t}$-tests revealed significant differences (unpaired t-test $\mathrm{p}<0.01$ ) for thalamus, habenula, caudoputamen, septo-hippocampal complex, and claustrum, and $\mathrm{p}<0.02$ for hypothalamus, basolateral amygdala, and nucleus accumbens.

F. Trial-aligned SVM decoder accuracy for whisker stim, odor stim, response, outcome and rule. $\mathrm{N}=1115$ units from 8 animals (PFC-VMS, left), and $\mathrm{N}=1770$ units from 9 animals (PFC-MDT, right).

G. Histograms traces of the fraction of cells modulated (rank sum $\mathrm{p}<0.01$ ) by trial outcome over trial-aligned timepoints and through learning stages for PFC-VMS (red) and PFC-MDT (green) neurons.

H. Trial-averaged activity histograms for PFC-VMS (left) and PFC-MDT (right) cells. Each cell's mean trace is normalized to its peak value, and cells are sorted by time of peak excitation (top) or inhibition (bottom).

I. Mean outcome difference (incorrect - correct) traces for PFC-VMS and PFC-MDT neurons. Each cell's mean difference trace is normalized to its peak value, and cells are sorted from most strongly preferring correct outcomes (top) to incorrect outcomes (bottom).

J. First four principal components for trial-averaged activity histograms in PFC-MDT (top) and PFC-MDT (bottom) neurons.

To examine the degree to which projection target specificity contributed to the functional heterogeneity 284 of in PFC neurons during set-shifting, we examined the functional properties PFC-VMS and PFC-MDT 285 neurons, two projection-defined PFC output populations whose target structures have both been implicated 286 in supporting cognitive flexibility in prior work (Block et al., 2007; Floresco et al., 2006; Marton et al., 2018). 287 Labeling of both populations within the same animals (Soudais et al., 2001; Tervo et al., 2016), using rAAV288 Cre labeling, revealed two largely non-overlapping cell types that were also spatially intermingled (Fig. 5A). 289 Despite their being spatially interspersed, double labeling of PFC-VMS and PFC-MDT neurons showed 290 little $(<5 \%)$ overlap (Fig. 5B). Examination of fluorescent axons in fluorescently labeled neurons from the 291 two groups (Fig. 5C-D) revealed two distinct populations. PFC-VMS neurons sent dense collateral 292 projections to the claustrum, septo-hippocampal complex, caudoputamen, and basolateral amygdala, but 293 not to MST. PFC-MDT neurons sent dense collateral projections to the habenula, hypothalamus and other 294 thalamic nuclei, but not to VMS Fig. 5E and S13).

Contrary to our expectations, the two populations showed a striking degree of overall similarity in their task-responsiveness. We used linear decoders to assess which task-related variables were represented in the population-level activity of the two cell types and found that both exhibited robust representation over similar time-courses for all five examined task features (Fig. 5F). Both populations showed modulation by trial outcome that emerged through successive learning stages (Fig. 5G). Similar distributions of neurons in each population were excited vs inhibited by trial onset (Fig. $\mathbf{5 H}$ ) and the two cells types each showed comparable distributions of correct-preferring and incorrect-preferring neurons (Fig. 5I). Principal component analysis showed similar trial-aligned temporal profiles for the main components (Fig. 5J), illustrating similar underlying structures accounting for the largest proportions of variance across the populations. Together, these results show that while individual PFC neurons are highly heterogeneous in their functional properties, efferent projection targets do not account for this functional heterogeneity in these two major projection subtypes. \\ Interference with feedback monitoring in PFC projection populations impairs set-shifting}


Spellman et al., 2020

309 The finding of durable representations of trial feedback signals over successive trials raised the 310 possibility that the task-critical role of the PFC might be feedback monitoring. However, the robust 311 representation of stimuli and task rule, particularly during stimulus presentation, tended to support the 312 prevailing model of PFC involvement in set-shifting, namely that PFC activity controls top-down, attention313 mediated biasing of rule-dependent action selection in the task. We next tested these possibilities using 314 projection-targeted optogenetics.

We used the soma-targeted anion-conducting channelrhodopsin stGtACR2 (Mahn et al., 2018) to inhibit PFC activity (Fig. 6A). Photoactivation of the channel across a 10-fold range of light intensities 317 produced strong silencing of spiking activity in extracellular recordings (Fig 6B). Next, we tested the 318 requirement of PFC activity for successful set-shifting performance in three temporally controlled 319 stimulation regimes: during trials $(0.5 \mathrm{~s}$ trial-ready cue period, $2.5 \mathrm{~s}$ stimulus presentation, and $\leq 1.5 \mathrm{~s}$ 320 response window), during the ITI (8-10s epoch triggered on response lick) following CG trials, or during the 321 ITI following ICG trials (Fig. 6C). Trial blocks (beginning with rule switch and ending with the animal 322 reaching criterion for the new rule) alternated light off/on.

323 Photoactivation of pan-neuronally-expressed stGtACR2 during the inter-trial interval following 324 incongruent trials impaired performance on ICG trials (6D) but not on CG trials (Fig. 6E). No effect of light 325 was seen for control tdTomato-expressing animals (Fig. 6D-E). This effect was also seen in animals 326 expressing stGtACR2 in PFC-VMS or PFC-MDT projection neurons (Fig. 6D-E). When light was delivered 327 following CG trials, no impairment was seen on ICG trial performance for either projection population (Fig.

328 6F), indicating that the impairment seen with post-ICG inhibition reflected an interference with prior trial 329 feedback, rather than preparation for the subsequent trial. The impairment on ICG trials that resulted from 330 photoactivation of PFC following ICG trials was seen both early and late in trial blocks (Table S16). 331 Together, these results confirm a critical role for post-trial activity in both PFC-VMS and PFC-MDT neurons 332 in enabling set-shifting.

333 Strikingly, neither PFC-VMS nor PFC-MDT activity was necessary for execution of the rule-guided 334 response, as no impairment was seen with photoactivation during ICG trials (6G). Failure to disrupt 335 performance by silencing during trials demonstrates that, even though PFC activity represents the task rule 336 and identities of the stimuli (Fig $\mathbf{2 F}, \mathbf{5 F}$ ), these representations are not critical for correct responding. In 337 light of this unexpected result, we sought another association area that might mediate rule-dependent 338 responding in real-time. We chose the posterior parietal cortex (PPC), which has been previously 339 implicated in cognitive flexibility (Fox et al., 2003; Prado et al., 2017), as well as in monitoring sensory 340 history (Akrami et al., 2018). Silencing of PPC during trials impaired performance on ICG trials but not on 341 CG trials (Fig. 6G), indicating that the PPC mediates responding in the task in a specifically rule-dependent 342 manner, possibly by an attentional mechanism.

\section{Feedback-related activity follows an anatomical gradient, independent of efferent target.}

345 The surprising similarity of both task-related activity and the task-critical function of the PFC-VMS and 346 PFC-MDT pathways left open the question of whether the functional heterogeneity of PFC neurons might 347 be explained by their laminar distribution. We therefore examined whether the relative locations of neurons 348 within the PFC imaging field affected task-related activity. As has been demonstrated in sensory cortex 349 (Smith and Kohn, 2008), temporal correlations across pairs of simultaneously-recorded neurons decayed 350 with distance (Fig. 7A), demonstrating an association between spatial proximity and temporal coactivation 351 within the context of the task.

352 To assess whether neurons' responsiveness to trials varied as a function of their distance from the pial 353 surface, we quantified the population variance of trial-averaged waveforms to capture the magnitudes of 354 neuron responses, including those both excited and inhibited at each time point. More deeply situated 355 neurons (further from pial surface) showed greater population variance (Fig. 7B), exhibiting more diverse 356 trial responses than more superficially located neurons. Greater response amplitude (absolute value of z357 scored activity) was seen in deeper neurons from both PFC-VMS and PFC-MDT populations Fig. 7C).

358 In addition to trial responsiveness, task-related information was heterogeneously distributed across the 359 cortical laminar axis, with more deeply situated neurons exhibiting greater selectivity for response and 

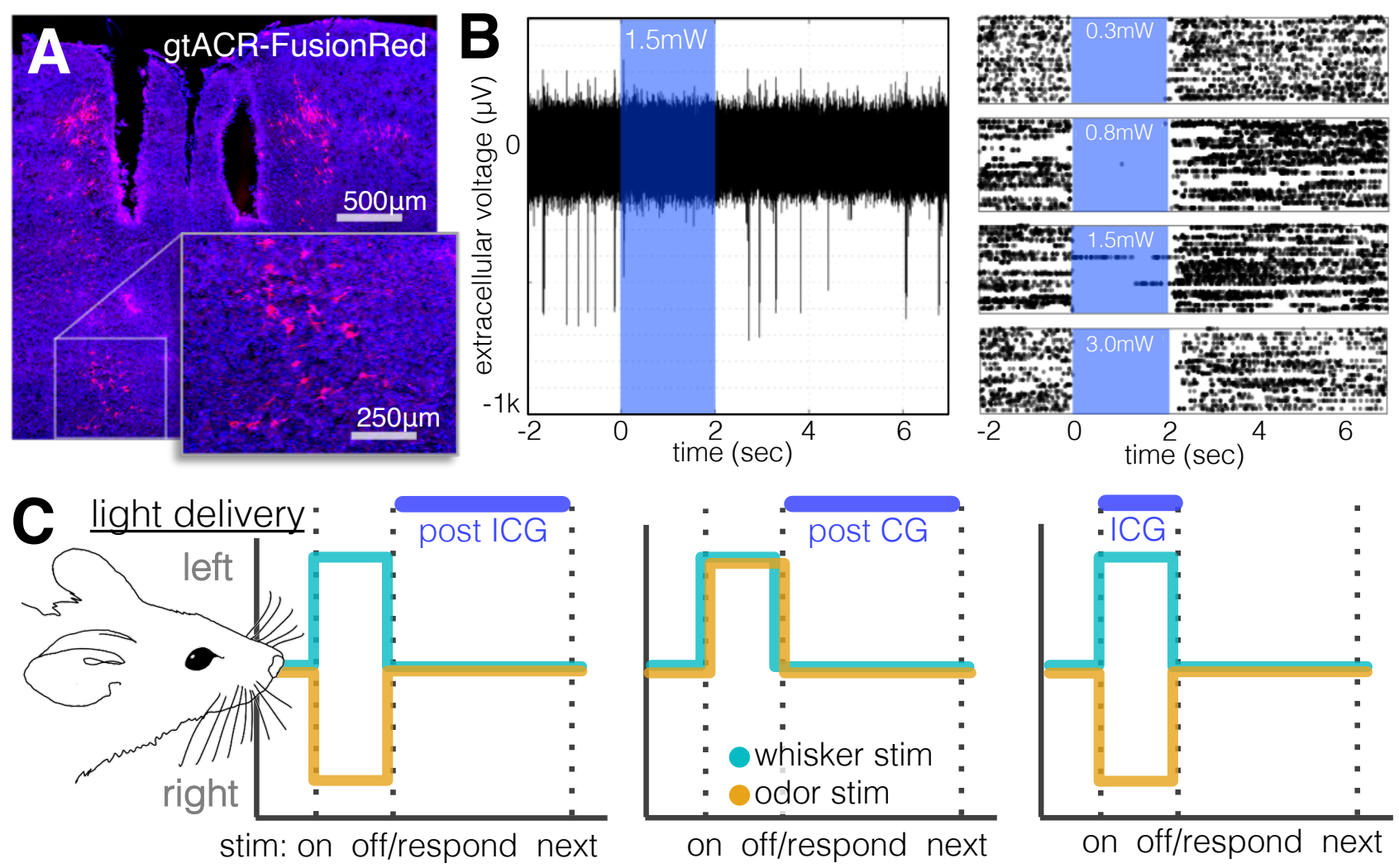

D post-ICG light
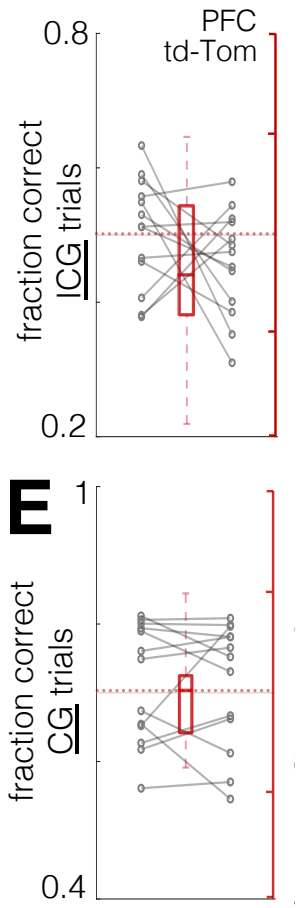

light: off on
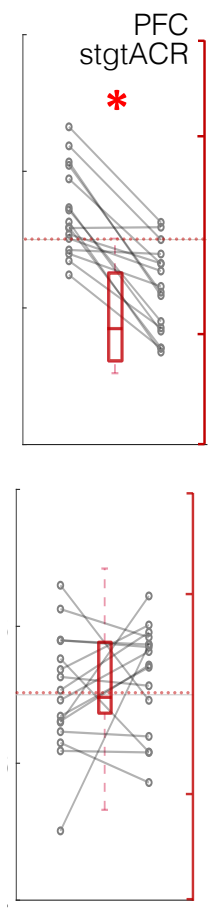

off on
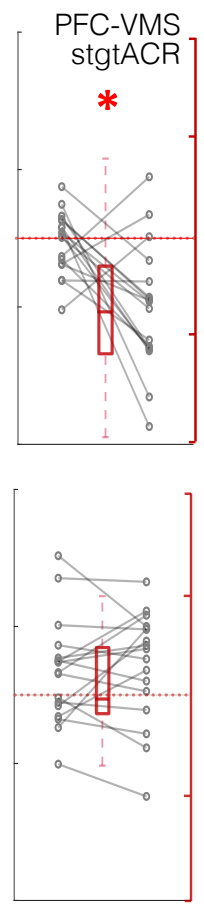

off on
F post-CG light

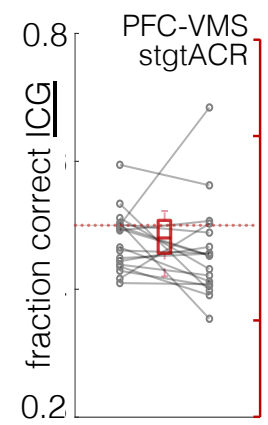

light: off on off on
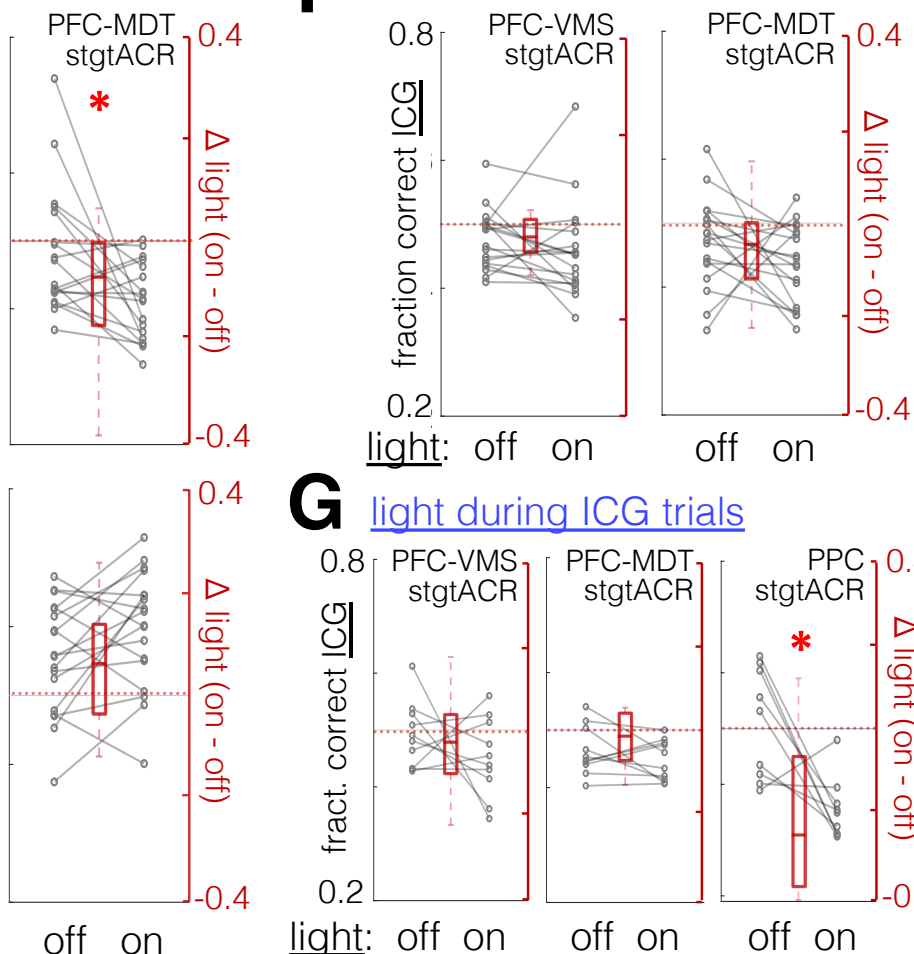

G

light during ICG trials

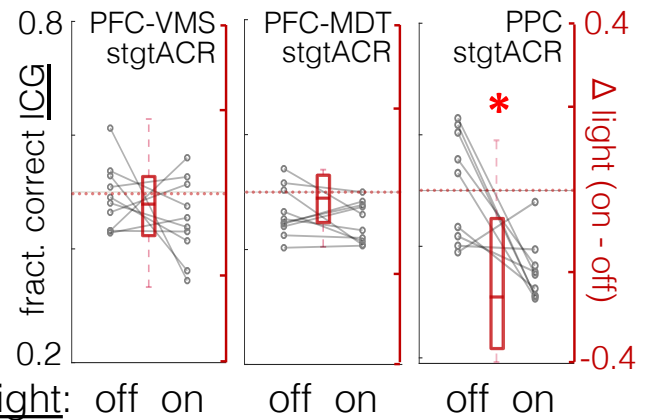


Figure 6: Set-shifting performance requires PFC-VMS and PFC-MDT activity following rule-informative trials

A. Cre-mediated expression of gtACR-FusionRed in PFC-VMS neurons, and fiber tracks from chronically implanted bilateral optical fibers (fixed dissue, DAPI in blue).

B. Electrophysiological demonstration of gtACR-mediated silencing. Left: a trace of spontaneous activity ( 7 seconds), with a 2-second light epoch interposed (1.5mW, $470 \mathrm{~nm}$ light, $200 \mu \mathrm{m}$ fiber, PFC, see Methods). Right: rasters of 50 sweeps for each of four light intensities: $0.3 \mathrm{~mW}$ (top), $0.8 \mathrm{~mW}, 1.5 \mathrm{~mW}$, and $3.0 \mathrm{~mW}$ (bottom).

C. Schematic diagram of light delivery conditions. Light was delivered during the inter-trial interval following incongruent trials, following congruent trials, or during incongruent trials.

D. Effect of light stimulation following incongruent trials on incongruent trial performance (fraction of trials correct). Left: viral control animals (td-Tomato expressed in PFC); second from left: animals with pan-neuronal expression of stgtACR2 in PFC; third from left: animals with stgtACR2 expressed in PFC-VMS projection neurons; right: animals with stgtACR2 expressed in PFC-MDT projection neurons. $N=13,16,17,18$ resp. Sign rank $p=0.4,0.0005,0.007$, 0.01 , resp.

E. Effect of light stimulation during ITIs following incongruent trials on congruent trial performance (proportion of trials correct). Left to right: as in (a) above. $\mathrm{N}=13,16,17,18$, resp. Sign rank $\mathrm{p}=0.9,0.5,0.9,0.07$, resp.

F. Effect of light stimulation during ITIs following congruent trials on incongruent trial performance. $\mathrm{N}=18,18$, resp. Sign rank $\mathrm{p}=0.1$ and 0.1 , resp.

G. Effect of light stimulation during trials (stimulus presentation + response window) on incongruent trial performance. $\mathrm{N}$ $=10$ and 10, resp. Sign rank $\mathrm{p}=0.4$ and 0.4, resp. Right: Effect of light delivered to posterior parietal cortex (PPC) during incongruent trials on incongruent trial performance $(\mathrm{N}=9$ animals, ranksum $\mathrm{p}=0.02$, opsin-negative control group, $\mathrm{N}=8$ animals, $\mathrm{p}=0.74$ ).

361

362

363

364

outcome during the ITI (Fig. 7D). General linear models for the neuron selectivities to response and outcome yielded significant coefficients for neuron depth but not for projection subtype. Because the distribution of these selectivities was positively skewed, we used a nonparametric test to compare sample distributions across groups of neurons from three terciles of pial depth and found that sample distributions differed significantly across depth for both response and outcome selectivities in both projection types, as evidenced by significantly different Kolmogorov-Smirnov scores between spatial terciles in Fig. 7E. We analyzed variance in response and outcome coding along the dorso-ventral axis, testing for differences between the prelimbic and infralimbic subregions, but we observed no such differences (data not shown). The findings of greater trial responsiveness and greater selectivity for trial response and outcome in deeper neurons led us to look for a potential mechanism by which deep neurons might exhibit stronger coding of task-critical variables than superficial neurons. To address whether deep and superficial neurons have differential inputs, we employed a rabies tracing approach, targeting EnvA G-deleted rabies-mCherry to PFC projection neurons in spatially restricted deep or superficial injections (Fig. 7F). We imaged histological sections from across much of the midbrain and forebrain (bregma AP $+3 \mathrm{~mm}$ to $-4 \mathrm{~mm}$ ) and annotated the locations of monosynaptic inputs to the PFC projection neurons. A comparison of the distributions of cells labeled by deep vs superficial injections, as a function of their distance from the PFC injection sites, revealed a significant difference, driven mostly by neurons located between $1 \mathrm{~mm}$ and $2 \mathrm{~mm}$ from the PFC injection sites (Fig. 7G). An analysis of the regions occupied by these neurons showed them to be most densely located in the caudo-ventral portion of the anterior cingulate cortex (ACC), and a comparison of cell densities from deep and superficial injections in this region showed a higher density of projections to deep PFC projection neurons in this region (Fig. 7G-H). Given that ACC has been identified as an area critical for both set-shifting behavior and reward-related feedback monitoring in previous studies (Bissonette et al., 2013; Hyman et al., 2017) this enriched input from caudo-ventral ACC to deep PFC projection neurons provides a potential source of input driving the stronger representation of trial feedbackrelated information seen in the deeper neurons. Further brain-wide differences in the distributions of inputs to deep and superficial PFC projection neurons can be explored in Supplementary Fig. S14.

As a further test of the association between laminar depth and stronger coding of response and outcome signals, we leveraged the selective viral tropism of two separate PFC-MDT projection populations (Fig. 7I), one layer 5 population, for which rAAV2 has strong tropism, heretofore referred to simply as PFCMDT and hereafter referred to as PFC(L5)-MDT, and one in layer 6, for which canine adenovirus (Cav2) has strong tropism, hereafter referred to as PFC(L6)-MDT (Collins et al., 2018). We used these two viruses as Cre expression vectors to selectively label the two populations with GCaMP6f, and compared their 

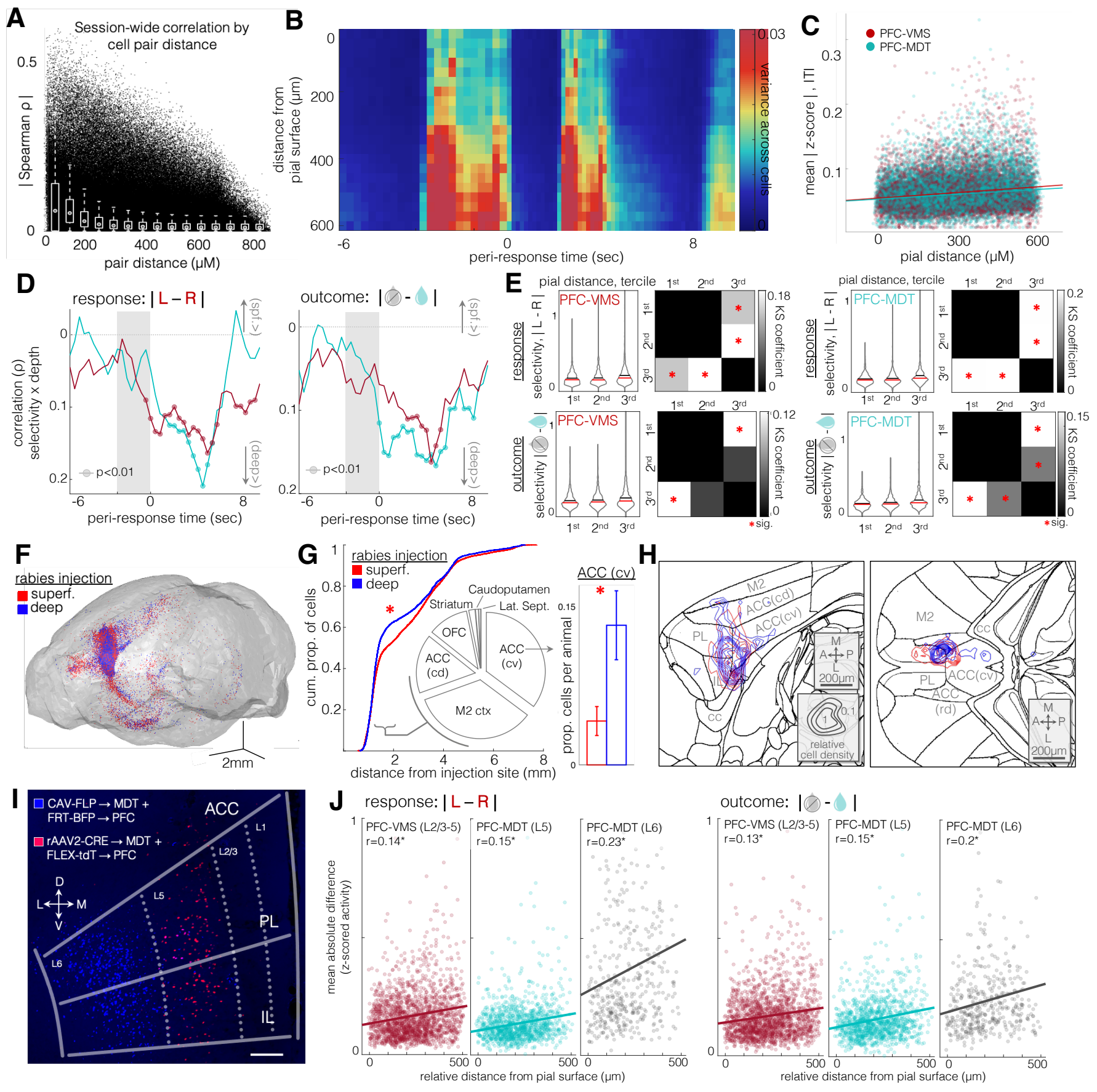

Figure 7: Post-trial feedback-related activity in projection populations is organized by a common topological gradient

A. Spatial distance of all simultaneously recorded cell pairs vs. temporal correlation. Correlations are partial correlations, controlling for changes in putative neuropil (temporal profile background component from CNMF-E source extraction algorithm). Spearman $\mathrm{R}$ for distance vs partial correlation is $-0.25, \mathrm{p}=0$ for $\mathrm{N}=1562607$ pairs from 4740 cells in 21 animals. White plots are box plots for $60 \mu \mathrm{M}$ bins.

B. Cross-neuron variance of trial-averaged activity, over trial-aligned timepoints and relative distance from pial surface. Warmer colors correspond with higher variance per spatiotemporal bin. Units are means of z-scored activity. Same cells as in (A).

C. Time-averaged data from (B), broken out by neuron and by projection cell type. Correlation of absolute values of $\mathrm{z}-$ scored activity with relative pial distance during ITI for PFC-VMS and PFC-MDT neurons. PFC-MDT Spearman $\rho=$ 0.16, $\mathrm{p}=8 \mathrm{e}-79,1770$ neurons, 9 animals; PFC-VMS Spearman $\rho=0.12, \mathrm{p}=3 \mathrm{e}-52, \mathrm{n}=1155$ neurons, 8 animals.

D. Correlations of feature selectivity with pial distance at trial-aligned timepoints. Left: response selectivity; right: outcome selectivity. Selectivity is defined as mean absolute difference in z-scored activity between conditions. Circles, Spearman $\mathrm{p}<0.01$. PFC-VMS in red, PFC-MDT in green (same units as in D). Note: $y$-axis is inverted so that "deep $>$ " values 
appear on the bottom.

E. Distributions of cell feature selectivity for response and outcome during ITI for PFC-VMS and PFC-MDT populations, binned by tercile of relative pial distance. Top row, response selectivity; bottom row, outcome selectivity; left column, PFC-VMS neurons; right column, PFC-MDT neurons. Red cross-bars are medians, black cross-bars are means. Binned spatial depths were tested for significant differences using a Kolmogorov-Smirnov fit test. For trial outcome selectivity across spatial depth, the KS Chi-square for PFC-MDT neurons was $23.09, \mathrm{p}=4 * 10^{\wedge}-05$; for PFC-VMS neurons, Chisquare was $19.3, \mathrm{p}=0.0002$. For response selectivity across spatial depth, KS Chi-square for PFC-MDT neurons was $30.2, \mathrm{p}=1 * 10^{\wedge}-06$; for PFC-VMS neurons, Chi-square 36.54, $\mathrm{p}=6^{*} 10^{\wedge}-08$. Checkered plots are coefficients from posthoc 2-sample Kolmogorov-Smirnov tests, comparing distribution similarity across all pairs of terciles. Red asterisks are comparisons with Bonferroni-corrected significance.

F. Composite brain volume from the rabies tracing experiment. Shown is a three-dimensional rendering of labeled starter cells in PFC, as well as brain-wide retrogradely-labelled afferent cells synapsing onto deep and superficial projection populations (superficial injections, $300 \mu \mathrm{m}$ from midline, $50 \mathrm{~nL}$; deep injections, $600 \mu \mathrm{m}, 50 \mathrm{~nL}$ virus). $\mathrm{N}=45421$ cells from 22 animals, 13 deep, 9 superficial injections. Starter cells were PFC-VMS (N=6 injections) and PFC-MDT (N=16 injections). Two way GLM revealed no significant differences in the number of cells labeled according to projection cell type $(\mathrm{t}=1, \mathrm{p}=0.3)$ or injection depth $(\mathrm{t}=1.1, \mathrm{p}=0.3)$.

G. A Kolmogorov-Smirnov goodness-of-fit test was used to compare distributions of cell density by distance from injection site. Red and blue traces are cumulative density plots for cells labeled by deep (blue) and superficial (red) injections (KS p-value 2.07e-26, n= 9752 and 4755 cells from superficial and deep injections in 9 and 13 animals, respectively). The regions immediately surrounding the injection site (PL, IL, and rostral ACC) were excluded, as they were likely to contain a large number of labeled starter cells. The largest difference in labeled cell density between deep and superficial injections (wherein the first-order derivatives of their CDF curves diverged) was observed in the range of $1 \mathrm{~mm}$ to $2 \mathrm{~mm}$ distance from the injection site. Labeled cells in this range were most dense in caudo-ventral ACC (35\%), secondary motor cortex (34\%), caudo-dorsal ACC (16\%), striatum (11\%), caudoputamen ( $2 \%)$, and lateral septum $(<1 \%)$. Of these, the subregion with the most total cells, caudo-ventral ACC, showed a significant difference in cell count by injection depth, with deep-injection animals showing a higher proportion of cells in cv-ACC than superficial-injection animals $(0.13+/-0.03$ vs $0.04+/-0.01$ of total per animal, $\mathrm{t}=2.2, \mathrm{p}=0.04)$.

H. Cell density contour plots for sagittal (left) and horizontal (right) cross-sections through the regions surrounding ACC. Concentric contours show summed density pooled across all animals (blue, deep-injection animals, red, superficialinjection animals), normalized within the cross-section shown (a.u.). Higher cv-ACC density in deep-injected neurons can be seen in both orientations. Relatively higher density in M2 cortex in superficial-injection animals did not meet significance.

I. Labeled subpopulations of PFC-MDT cells with selective tropism for rAAV2 (predominantly located in layer 5, hereafter referred to as PFC(L5)-MDT neurons) and Cav (predominantly located in layer 6, hereafter PFC(L6)-MDT neurons). Dual labeling was achieved by co-injecting a viral mixture of rAAV2-Cre and Cav-FLP in MDT, and a second viral mixture of FLEX-tdT and FRT-BFP in PFC.

J. Pial depth and feature selectivity for the three projection subtypes. Right: Kruskal Wallis ANOVA of cell selectivity for response across cell types: Chi-square statistic $=440, \mathrm{p}=2 * 10^{\wedge}-96$. Post-hoc GLM testing of cell type by pial distance showed significant group differences between PFC-VMS vs PFC(L6)-MDT ( $\mathrm{t}=3.4, \mathrm{p}=0.0007)$, and for PFC(L5)-MDT vs PFC(L6)-MDT ( $\mathrm{t}=3.9, \mathrm{p}=0.0001)$, but not for PFC-VMS vs PFC(L5)-MDT ( $\mathrm{t}=1.0, \mathrm{p}=0.3)$. Post-hoc testing of withingroup effect of pial distance on cell selectivity for response and trial outcome revealed parallel effects (no cell-type-bypial-distance interaction effects) for all three groups. Spearman $\mathrm{R}$ for pial distance by response selectivity was significant for PFC-VMS ( $\left.\mathrm{r}=0.14, \mathrm{p}=8 \times 10^{\wedge}-9\right)$, PFC(L5)-MDT ( $\left.\mathrm{r}=0.15, \mathrm{p}=1 * 10^{\wedge}-7\right)$, and PFC(L6)-MDT ( $\mathrm{r}=0.23$, $\mathrm{p}=1^{*} 10^{\wedge}-6$ ). Left: Kruskal Wallis non-parametric anova of cell selectivity for trial outcome (mean absolute difference in z-scored activity between correct and incorrect trials during the subsequent inter-trial interval) across cell types: Chisquare statistic $=134, \mathrm{p}=10^{\wedge}-30$. Post-hoc GLM testing of cell type by pial distance, however, showed no group differences between PFC-VMS vs PFC(L5)-MDT ( $\mathrm{t}=0.5, \mathrm{p}=0.6)$, between PFC-VMS and PFC(L6)-MDT ( $\mathrm{t}=1.25$, $\mathrm{p}=0.2)$, or between PFC(L5)-MDT and PFC(L6)-MDT $(\mathrm{t}=1.6, \mathrm{p}=0.1)$. Spearman $\mathrm{R}$ for pial distance by trial outcome selectivity was also significant for each group: PFC-VMS ( $\left.\mathrm{r}=0.13, \mathrm{p}=2^{*} 10^{\wedge}-8\right), \operatorname{PFC}(\mathrm{L} 5)-\mathrm{MDT}\left(\mathrm{r}=0.15, \mathrm{p}=3^{*} 10^{\wedge}-7\right)$, and PFC(L6)-MDT ( $\left.\mathrm{r}=0.2, \mathrm{p}=4 * 10^{\wedge}-5\right)$. PFC-VMS: $\mathrm{N}=1155$ cells from 8 animals; PFC(L5)-MDT: $\mathrm{N}=1770$ cells from 9 animals; PFC(L6)-MDT: $\mathrm{N}=430$ cells from 8 animals.

selectivity for response and outcome during the ITI. If greater distance from the pial surface predicts stronger selectivity for response and outcome, layer-6 PFC-MDT neurons should show stronger modulation by these two variables compared with layer-5 PFC-MDT neurons or PFC-VMS neurons and should also exhibit a superficial/deep spatial gradient within the neuronal subtype. PFC(L6)-MDT neurons indeed showed greater selectivity for either PFC(5)-MDT or PFC-VMS neurons, as well as exhibiting the same within-population spatial gradient seen in PFC(L5)-MDT and PFC-VMS neurons (Fig. 7J). Together, these 
Spellman et al., 2020

400 results reveal a significant source of explanatory variance for the functional heterogeneity seen in PFC

401 neurons during set-shifting. These neurons form a topological gradient, with deeper neurons exhibiting

402 more trial responsiveness and stronger selectivity for trial response and outcome, potentially driven by

403 differences in their afferent connectivity profiles and in particular by differential innervation by the caudo-

404 ventral ACC.

405

\section{Discussion}

407 We began this study with the aim of more clearly resolving the circuit-level mechanisms by which 408 prefrontal activity uses attentional sets to provide context-dependent modulation of sensorimotor 409 processing in set-shifting tasks. The attentional set model of prefrontal involvement in cognitive flexibility, 410 built upon decades of influential literature with thousands of citations, frames the prefrontal cortex as a 411 mediator of top-down cognitive control, which, in the context of an attentional set-shifting task, would 412 facilitate the filtering of multimodal sensory inputs and the biasing of corresponding motor responses in 413 accordance with context-dependent task rules. Instead, what we present is a very different model for PFC 414 involvement in set-shifting. Rather than modulating attention in real-time, PFC output neurons serve to 415 integrate and maintain representations of recent behaviors and their consequences.

416 While some prior pharmacological silencing studies found PFC activity to be critical for recall but not 417 acquisition of rule switching, and that PFC activity was not critical for rule switches once the rules became 418 familiar (Rich and Shapiro, 2007), those experiments were performed in spatial tasks with changing 419 navigation rules, which may not explicitly engage attention or suppression of irrelevant sensory cues in the 420 same way that a cross-modal set-shifting task does. This difference may account for the result of the 421 muscimol experiment in this study, which does find PFC activity to be necessary for switch acquisition, as 422 well as for continued performance of rule shifts after overtraining. We note that the timing of rule shifts, 423 triggered by criterion performance, means that anticipation of these rule shifts cannot be ruled out. 424 However, such a strategy would necessarily involve both acquisition of the new rule as well as anticipation 425 of the change, and it would therefore not eliminate the requirement for any of the key cognitive components 426 of the task. Moreover, the evidence of rule-guided performance (Figure 1F) and the failure of animals to 427 immediately abandon the previous rule following a rule shift (Figure 1G) argue against such a confound. 428 We also note that, while the training sequence used to expose animals to multiple task rules and stimulus 429 exemplars is based on standard protocols that have been well validated by earlier set-shifting studies 430 (Birrell and Brown, 2000; Bissonette et al., 2008; Tait et al., 2014), we did not specifically test the effect of 431 each training step on the physiology of behavioral strategy used in the final task. Nevertheless, the 432 essential cognitive requirements of the task, as well as the performance data analyzed both with and 433 without perturbation (Figure 1C-G), strongly support the conclusion that the protocol achieves comparable 434 results and engages the same cognitive strategy as the protocols on which this task is based.

435 The finding of retrospective and persistent representation of response and outcome signals, modulated 436 by the demands of the task, are not without precedent. For example, Sul et al found that response on a 437 two-armed bandit task was not represented in PFC activity in advance of the choice (Sul et al., 2010), but 438 this was in an explicit test of reinforcement learning and choice valuation. The attentional set model of PFC 439 involvement in set-shifting predicts that representations of rule, stimuli, and corresponding responses 440 should be activated during decision-making in order to modulate motor responses in downstream 441 structures, such as striatum, thalamus and periaqueduct grey matter.

442 Responsibility for modulating attention, possibly by active suppression of irrelevant stimuli, may be 443 played by regions with known involvement in multisensory integration - it was this hypothesis that led us 444 to inhibit PPC activity during stimulus presentation. This perturbation did interfere with performance, on 445 ICG but not on CG trials, lending support to the hypothesis.

446 The finding that response and outcome are not natively encoded as functions of the overt discrimination 447 behavior, but emerge only once the task rules require repeated/continuous reinforcement learning, lend 448 further support to the conclusion that the role of PFC in this task is to facilitate rule switching by monitoring 449 feedback. These findings build on a body of research showing that feedback signals within PFC are 450 modulated by expectation and by cognitive states, including attention (Smout et al., 2019). Extensive 
451 research in feedback-related negativity (Hauser et al., 2013) and reward prediction error (Garrison et al.,

452 2013) support the idea that feedback-related signals in prefrontal areas are dynamically mediated by

453 ongoing task demands and are disrupted in disorders in which set-shifting is also disrupted (Toyomaki et

454 al., 2017).

455 It is noteworthy that, despite not being critical for task performance, stimulus information is nevertheless

456 robustly expressed in neural activity during stimulus presentation. The observation of strong task-related

457 activity that is nevertheless evidently not critical for task-critical performance, raises the question of what

458 adaptive information-processing purpose this activity may serve, and it underscores the need to assess

459 the function of neural activity through perturbation, rather than assuming a critical role for all task-related 460 signals.

$461 \quad$ Future work will seek to elucidate potential neuromodulatory and plasticity-related mechanisms behind

462 these signals. Given the high density of input from the cholinergic nuclei of the basal forebrain that

463 synapses on to these projection neurons (Fig. S14-B), and the established relationship between the central

464 acetylcholine neuromodulatory system and attention-mediated behavior (Ljubojevic et al., 2014; Proulx et

465 al., 2014), the possibility that acetylcholine plays a key role in mediating these feedback monitoring signals

466 remains strong.

\section{Acknowledgements}

T.S. is supported by $\mathrm{NIH}(1 \mathrm{~K} 99 \mathrm{MH} 117271)$, the Brain and Behavior Research Institute, and the Leon Levy Foundation. C.L. is supported by NIH (R01 MH109685, MH118451, MH123154) and by the Rita Allen Foundation, Hope for Depression Research Foundation, Pritzker Neuropsychiatric Disorders Research Foundation, and the Foundation for OCD Research. The authors thank S. Fusi, F. Stefanini, and L. Grosenick for consultation on the planning and implementation of data analyses.

\section{Author Contributions}

$\mathrm{CL}$ and TS designed the behavioral task and planned the study. TS and MS performed the injection and implantation surgeries and gathered the behavioral data. TS and MS performed the neural recordings. TS analyzed the behavioral and neural data. JK performed the TCA analysis. GMN assisted with the EnvA Gdeleted rabies experiment. TS and CL wrote and edited the manuscript.

Declaration of Interests: Competing interests: The authors declare no competing interests.

\section{EXPERIMENTAL MODEL AND SUBJECT DETAILS}

490 Male C57BL/6 mice (Jackson Labs) were used for all experiments, aged 6-10 weeks at first use. Mice were 491 housed in a Weill Cornell Medical College facility and were maintained on a 12-hour light-dark cycle. Except 492 when water-restricted for the purpose of behavioral training and testing, all mice were given ad libitum 493 access to food and water. Littermates underwent prism or fiber implant surgeries within the same week, 494 and mice were group-housed with littermates with the same surgery status. All procedures were approved 495 by the Weill Cornell Medicine IACUC. Sample sizes for each experiment were determined using power 496 analysis estimates computed in Matlab, based on anticipated effect sizes that were estimated from 497 previously published reports whenever they were available, and were powered to detect moderate, 498 biologically meaningful effect sizes. 


\section{METHOD DETAILS}

\section{Surgery}

502 Animals were placed inside a flow box and anesthetized with isoflurane gas (2\%) until sedated, at which 503 point they were placed in a stereotax and maintained on $0.5 \%$ isoflurane for the duration of the surgery. 504 Scalp hair was trimmed away, and a midline incision was made using fine surgical scissors (Fine Science 505 Tools), exposing the skull. The periosteum was bluntly dissected away and bupivacaine $(0.05 \mathrm{~mL}, 5 \mathrm{mg} / \mathrm{kg})$ 506 was topically applied. For prism implantation, a large rectangular craniotomy was made above left PFC, 507 extending from $1.5 \mathrm{~mm}$ anterior to $3.7 \mathrm{~mm}$ anterior, and from $2.0 \mathrm{~mm}$ lateral (left) to $0.2 \mathrm{~mm}$ lateral (right, 508 across midline).

509 A 0.5-mm burr (Fine Science Tools) and a high-speed hand dental drill (Osada) were used, taking great 510 care not to compress brain tissue or damage the sagittal venous sinus. In the event of venous bleeding, 511 Gelfoam (Pfizer) was applied to the dura surface to accelerate clotting. Gentle irrigation with phosphate512 buffered saline (137 mM NaCl, $27 \mathrm{mM} \mathrm{KCl}, 10 \mathrm{mM}$ phosphate buffer, VWR) was used to clear debris at 513 regular intervals. The dura beneath the craniotomy was removed using the tip of a $26 \mathrm{~g}$ insulin syringe 514 (VWR) and fine forceps (Fine Science Tools).

515 Chronically implanted microprisms (1.5mm X 1.5mm X 3mm; M/L,A/P,D/V), from OptoSigma (BK7 516 borosilicate glass with aluminum hypotenuse and silicon dioxide coating), were implanted at a depth of $5172.3 \mathrm{~mm}$ ventral to brain surface using a stereotaxic micromanipulator (Kopf). During implantation, the prism 518 was held in place using vacuum suction via an 18G blunt needle. As in previous studies (Andermann et al., 519 2013; Low et al., 2014) minimal reactive gliosis was seen in the coronal imaging field, and maximum 520 calcium-mediated fluorescence was seen $50-150 \mu \mathrm{m}$ past the prism face; therefore imaging planes were 521 confined to this depth.

522 For PFC-VMS and PFC-MDT projection targeting, an additional craniotomy was made at $1.25 \mathrm{~mm} / 1.25 \mathrm{~mm}$ $523 \mathrm{~A} / \mathrm{L}$ or $1.2 \mathrm{~mm} / 0.35 \mathrm{~mm} \mathrm{P} / \mathrm{L}$, respectively. All head-fixed animals received custom-machined stainless steel 524 head plates affixed to skull surface with Metabond dental cement. Head plates featured a circular central 525 aperture centered around the imaging field ( $9 \mathrm{~mm}$ I.D.), with right and left securing arms (25mm total width) 526 that accommodated $0-80$ socket screws (0.38g in total). Sterile eye lubricant (Puralube, FischerSci) was 527 administered to prevent corneal drying, and a microwavable heating pad (Snugglesafe) was used to 528 maintain body temperature. Metacam (1 mg/kg, i.p.) was administered after surgery as a prophylactic 529 analgesic.

\section{Viral Transduction}

532 AAV of titer exceeding $10^{12} \mathrm{vg} / \mathrm{ml}$ (Vector Biolabs, UNC Vector Core and Addgene) was used to package 533 the plasmids. For imaging experiments, AAV1-hSyn-GCaMP6f or AAV1-hSyn-DIO-GCaMP6f (Chen et al., 534 2013) was targeted to PFC. Injection coordinates for PFC were $1.75 \mathrm{~mm}$ anterior. Two parallel injection 535 tracks were made at $0.2 \mathrm{~mm}$ and $0.5 \mathrm{~mm}$ lateral, and in each of these tracks, two D/V sites received 536 infusions, at $2.0 \mathrm{~mm}$ and $1.5 \mathrm{~mm}$ ventral to brain surface. For rAAV2-Cre projection targeting, VMS injections 537 were delivered at $1.25 \mathrm{~mm} / 1.25 \mathrm{~mm} / 4.7 \mathrm{~mm} \mathrm{~A} / \mathrm{L} / \mathrm{V}$, and MDT injections at $1.2 \mathrm{~mm} / 0.35 \mathrm{~mm} / 3.2 \mathrm{~mm} \mathrm{P} / \mathrm{L} / \mathrm{V}$. 538 Hamilton syringes and beveled 36G or 33G NanoFil needles (WPI) were used, and at each site, the needle 539 was allowed to sit $5 \mathrm{~min}$ to allow for tissue settling before infusion. Virus was infused at a rate of $50 \mathrm{~nL} / \mathrm{min}$, 540 for a total of $250 \mathrm{~nL}$ per site.

541 For the dual labeling tracing experiment (Fig. 5B), rAAV2-mNeuroGFP was injected in MDT (500nL), 542 rAAV2-Cre was injected in VMS (500nL), and AAV1-NLS-tdTomato was injected in PFC (500nL).

\section{Muscimol silencing}

544 Animals received chronically implanted 26G bilateral stainless steel guide cannulae (Plastics One), 545 implanted at $1.75 \mathrm{~mm} / 0.35 \mathrm{~mm} / 1 \mathrm{~mm} \mathrm{~A} / \mathrm{L} / \mathrm{N}$. After undergoing the training and task transition sequence up 546 to and including Reversal (see behavioral training protocol below), animals underwent muscimol infusion. 
547 In a familiar cage, bilateral internal infusion cannulae were inserted into the guide cannulae, protruding

$5480.5 \mathrm{~mm}$ from the end of the guide cannulae, and were left for $5 \mathrm{~min}$ to allow tissue to settle. Muscimol

$549(1 \mu \mathrm{g} / \mu \mathrm{L})$ or physiological saline $(0.9 \%)$ was infused at a rate of $50 \mathrm{~nL} / \mathrm{min}$, for a total of $0.25 \mu \mathrm{L}$. Five minutes

550 after completion of the infusion, the internal cannulae were removed and the mouse immediately began

551 behavioral testing.

\section{Optogenetic implantation and stimulation}

553 Bilateral fibers were implanted over PFC (Thorlabs dual fiber cannulae, $700 \mu \mathrm{m}$ center-to-center spacing, $554200 \mu \mathrm{m}$ core) at $0.35 \mathrm{~mm}$ lateral, 1.75 anterior, $1.2 \mathrm{~mm}$ ventral to brain surface. In the posterior parietal cortex 555 inhibition cohort, in order to cover the medial-lateral extent of PPC, a dual-fiber cannula $(700 \mu \mathrm{m}$ center-to556 center spacing) was placed over each hemisphere, so that both right and left PPC each had two $200 \mu \mathrm{m}-$ 557 core fibers. The dual fiber cannulae were positioned on the brain surface with the dura intact, at a ML angle 558 of $10^{\circ}$ away from midline, with the medial fiber at AP $-1.8 \mathrm{~mm}, \mathrm{ML}+1.0 \mathrm{~mm}$, and the lateral fiber at AP $5591.8 \mathrm{~mm}, \mathrm{ML}+1.7 \mathrm{~mm}$. Light was delivered via Thorlabs M470F3 fiber-coupled LED, 1.5-3mW light power 560 from each fiber. In the trial-concurrent stimulation condition, photoactivation was initiated at the same time 561 as the $500 \mathrm{~ms}$ trial-ready cue and persisted through the $2.5 \mathrm{~s}$ stimulus presentation epoch until either a lick 562 terminated the trial or the $1.5 \mathrm{~s}$ response period expired. In the inter-trial interval stimulation conditions, 563 photoactivation was initiated concurrently with the end of the trial (first lick within the response window or 564 upon the conclusion of the 1.5s response window without a lick) and persisted through the 8-10s post-trial 565 epoch, terminating with the onset of the subsequent trial-ready cue. In the experiment testing the effects 566 of inhibition during the beginning of the inter-trial interval, the photoactivation was initiated concurrently 567 with lickspout selection and persisted for 4.5 seconds before terminating; in the experiment testing the 568 effects of inhibition during the end of the inter-trial interval, photoactivation was initiated beginning at 4.5 569 seconds following lickspout selection, persisting for the remainder of the 8-10s inter-trial interval. Light was 570 delivered in alternating trial blocks, beginning with the second block of the session (no light delivered in the 571 initial block). Sessions in which animals failed to reach criterion on the initial block were re-run until the 572 animal reached criterion.

\section{2-photon imaging}

574 2-photon calcium imaging (Denk et al., 1990; Nakai et al., 2001) was performed via an Olympus 10x 0.6NA 575 objective, with $8 \mathrm{~mm}$ working distance. All images were acquired using a commercial two-photon laser576 scanning microscope (Olympus RS) equipped with a scanning galvanometer and a Spectra-Physics Mai 577 Tai DeepSee laser tuned to $920 \mathrm{~nm}$, operating at $300-500 \mathrm{~mW}$. Fluorescence was recorded through gallium 578 arsenide phosphide (GaAsP) detectors using the Fluoview acquisition software (Olympus) using a green 579 light emission bandpass filter (Semrock). Imaging sessions began by performing an isosbestic anatomical 580 scan (810nm 2P excitation light) to aid in relocating the same sites over multiple sessions. Calcium signals 581 were acquired at $256 \times 130$ pixel resolution, covering a $1500 \mu \mathrm{m} \times 760 \mu \mathrm{m}$ field of view, with a $\mu \mathrm{m} /$ pixel ratio 582 of 5.85 . The scan time was $346 \mathrm{~ms}$, with a frame rate of $2.89 \mathrm{~Hz}$. All calcium imaging experiments occurred 583 in awake mice. For analysis of SEDS sessions, sessions were concatenated using CellReg (Sheintuch et 584 al., 2017) and non-rigid spatial transformation. Neurons were modeled with a maximal centroid distance of

$58515 \mu \mathrm{m}$ and a threshold correlation of 0.65 . Z-scoring of deconvolved activity traces was performed prior to 586 concatenating across sessions. SEDS neural data sets for each of 21 animals are comprised of a median 587 of 3 sessions (2.5,4 upper, lower quartiles), 1462 trials (1034, 1667 upper, lower quartiles), and 11 set 588 shifts (8.5, 17.5 upper, lower quartiles).

\section{Image processing}

590 Videos were motion-corrected using NoRMCorre (Pnevmatikakis and Giovannucci, 2017) implemented in 591 Matlab (Mathworks), and a constrained non-negative matrix factorization-based source extraction method 592 was used to denoise, deconvolve and demix the videos to extract neural traces (using the extensively 593 validated CNMF-E package (Pnevmatikakis et al., 2016) with OASIS signal deconvolution (Friedrich et al., 594 2017). Sources were well separated from both neighboring sources and surrounding neuropil, as assessed 595 by performing PCA analysis of putative source and surrounding pixels over time and quantifying their 
596 isolation distances (Schmitzer-Torbert et al., 2005; Stringer and Pachitariu, 2019). The resulting traces

597 were deconvolved calcium traces corresponding with estimated event rates, which were then z-scored over

598 the full session to normalize. Calcium signals from sequential sessions were concatenated using non-rigid

599 co-registration of spatial cell footprints using CellReg (Crowe and Ellis-Davies, 2014; Sheintuch et al.,

600 2017), (see Fig. S3-S10 for example traces and quality control metrics).

601

\section{Behavioral Training and Testing Protocols}

603 Animals recovered 14 days from surgery before being placed on water restriction for four days, after which 604 they typically drank 1-1.2mL/day. Behavioral training and testing procedures were carried out 5 days per 605 week, with each animal undergoing one session per day. Animals underwent two days of hand-feeding, in 606 which they were handled for up to ten minutes by the experimenter while receiving water from a $1 \mathrm{~mL}$ 607 syringe with a rounded stainless steel gavage needle.

\section{Habituation-1}

609 Following two days of hand-feeding, animals underwent a Habituation-1 session in the behavioral 610 apparatus, which consisted of an aluminum restraint tube with dual lickspouts positioned at one end. The 611 restraint tube was $27 \mathrm{~mm}$ in diameter, a width calibrated to allow the animal to groom and adjust its posture 612 during sessions but which prevented significant lateral or vertical body movement. During Habituation-1, 613 lickspouts were alternately armed so that a single lick would trigger delivery of a 3uL water bolus, and the 614 identity of the armed lickspout changed every 1-4 trials, with a 1.5s timeout after each bolus delivery. This 615 alternating schedule forced the animal to explore both lickspouts equally to maximize rewards. Animals 616 would periodically venture out of the restraint tube to explore the behavior chamber, at which point the 617 experimenter would guide them back into the tube by hand. The session terminated when the animal 618 stopped licking for $2 \mathrm{~min}$, and the animal was considered to have passed this stage when it had consumed $619500 \mu \mathrm{L}$ in a session.

\section{Habituation-2}

621 After passing Habituation-1, animals underwent a Habituation-2 stage, which consisted of the same 622 lick/dispense schedule as Habituation-1, but during which the animal was head-restrained for the first time. 623 Here again, the animal needed to consume $500 \mu \mathrm{L}$ to pass.

\section{Habituation-3}

625 After Habituation-2, animals underwent Habituation-3, in which lickspouts alternated every 20 lick/delivery 626 trials, to establish left/right trial blocks. As with Habituation-1 and -2 , animals were considered to have 627 passed this stage after consuming $500 \mu \mathrm{L}$ of water.

\section{Shaping-1}

629 After this, animals underwent a Shaping-1 session, which introduced the trial structure to be used for the 630 rest of the sessions: a 500ms white noise trial-start cue was presented, followed by a 2.5s stimulus (whisker 631 or odor, depending on the training sequence, which was counter-balanced across animals in the calcium 632 imaging experiment), followed by a $\leq 1.5$ s response window, during which the correct lickspout was armed. 633 When the response rate fell to between 0.3 and 0.6 within a 10 -trial moving window, reward would be 634 dispensed regardless of whether the animal licked, in order to promote licking. Trials were blocked (20 635 right, 20 left), and a lick to the incorrect side would not terminate the trial; the animal was allowed to continue 636 until it licked the correct spout. At the start of each trial block, a single 3uL reward was dispensed at the 637 now-rewarded spout. Criterion for passing Shaping-1 was $500 \mu \mathrm{L}$ consumed.

\section{Shaping-2}

639 Shaping-2 differed from Shaping-1 in that trials were randomized between sides rather than blocked. 640 Criterion here, and for all remaining sessions up to SEDS, was reaching $80 \%$ correct within a 30 -trial 
Spellman et al., 2020

641 moving window, and simultaneously performing above $50 \%$ on both left and right trials, at any point within

642 the session.

\section{Direction bias correction}

644 To counteract animals' tendency to sporadically exhibit stereotyped direction bias, left/right stimulus 645 probability was dynamically computed as a function of the animal's concurrent direction bias coefficient. A 646 10-trial moving window was populated with a [-1] for each incorrect left response, a [1] for each incorrect 647 right response, and a [0] for each correct response. For each trial, the coefficient was the mean over the 64810 previous trials. A coefficient absolute value between 0 and 0.25 resulted in $50 \%$ left-right stimulus 649 probability; an absolute value between 0.25 and 0.75 resulted in a $33 \%$ chance of a stimulus toward the 650 direction of bias; an absolute value between 0.75 and 1 resulted in a $17 \%$ chance of a stimulus toward the 651 direction of bias. Trial runs with lick bias coefficients greater than 0.75 were therefore excluded from 652 analysis.

\section{Training on discrimination and cognitive shifting tasks.}

654 Stimuli: Of the 21 mice included in the imaging experiment, 14 underwent initial training with whisker 655 stimuli, and 7 underwent initial training with odor stimuli. Subsequent comparison of stimulus encoding did 656 not indicate any discernible differences between these two groups following the extradimensional set-shift. 657 It should be noted that the hearing range of mice is $1-80 \mathrm{kHz}$ (Turner et al., 2005), which falls outside the 658 range of the stimuli used to vibrate the whiskers.

659 For animals undergoing SD with whisker stimuli, stimuli consisted of a $35 \mathrm{~Hz}$ sinusoidal stimulus and a $660155 \mathrm{~Hz}$ sinusoidal stimulus. The $35 \mathrm{~Hz}$ and $155 \mathrm{~Hz}$ stimuli were delivered bilaterally. These stimuli were 661 generated by a pair of miniature base-frequency audio speakers (2" diameter) coupled to a pair of plastic 662 funnels which served to condense the sound waves into a $5 \mathrm{~mm}$ diameter compression wave (75 dB). These 663 stimuli produced oscillatory deflections of a majority of whiskers on the order of $10^{\circ}$, and active whisking 664 was routinely observed during these epochs.

665 Odor stimuli consisted of murine-appetitive oil extracts. For the 7 mice undergoing initial training with odor 666 stimuli, these stimuli were olive oil and sesame oil. The odor port was positioned 5mm below the animal's 667 nostrils, with the airflow directed up toward the nostrils. Air was delivered through this port at a rate of 2.5 668 liters per minute through 1/32" inner-diameter polytetrafluoroethylene tubing. Outside of the odor stimulus 669 epochs, clean air was delivered continuously through the odor port. At the onset of the odor stimulus epoch, 670 air was rerouted through chambers containing the oil extracts, so that clean air was completely displaced 671 by air from the oil container within $30 \mathrm{~ms}$.

672 Each trial began with a $500 \mathrm{~ms}$ audible white noise $(2 \mathrm{kHz}-17 \mathrm{kHz})$ to indicate the start of a trial. Immediately 673 following this cue, a whisker and/or odor stimulus (depending on the task phase as outlined in the table in 674 Figure 1 and descriptions below) were presented for $2.5 \mathrm{sec}$. The animal was permitted to lick the water 675 spouts freely during stimulus presentation, though these anticipatory licks did not terminate the stimulus or 676 trigger reward. Following the $2.5 \mathrm{sec}$ stimulus delivery, there was a response window of up to $1.5 \mathrm{sec}$ during 677 which a lick to either port would end the trial, trigger delivery of a $3 \mu \mathrm{L}$ water droplet (on correct trials), and 678 begin the inter-trial interval. As shown in Figure 1B, licking toward the ultimately chosen lick port began 679 well before the termination of the stimulus, and $95 \%$ of responses came within the first $0.346 \mathrm{~ms}$ of the 680 response window, a latency that corresponded with the first frame of imaging following stimulus termination.

681 Simple Discrimination (SD): For the 14 mice undergoing initial training with whisker stimuli, on each trial 682 a bilateral whisker vibration stimulus (one of two possible patterns) was delivered. Through water reward683 mediated reinforcement, mice learned to lick the left lick port in response to the $35 \mathrm{~Hz}$ stimulus and the right 684 lick port in response to the $155 \mathrm{~Hz}$ stimulus. For the 7 mice undergoing initial training with odor stimuli, 685 animals learned to lick left in response to an olive oil odorant and right in response to a sesame oil odorant. 686 Sessions consisted of up to 350 trials, and sessions were terminated early if an animal failed to respond to 687 ten consecutive trials. An animal was considered to have reached criterion for the SD phase if at any point 688 during a session it reached $80 \%$ correct performance, and simultaneously $\geq 50 \%$ correct on both left and 
689 right trials, within a 30-trial moving window. The SD phase tests the animal's ability to learn a stimulus-

690 reward contingency and discriminate between two stimuli from the same dimension (i.e. two odors or two

691 whisker stimuli). When criterion was reached, the current session continued to its end (up to 350 trials),

692 and the animal was moved on to the subsequent task phase on the following session.

693 Compound Discrimination (CD): An irrelevant stimulus (odor stimulus for mice initially trained on whisker

694 stimuli and vice versa) was added on each trial. Both relevant and irrelevant stimuli were randomly and

695 independently chosen for each trial. The same criterion standard was used as with SD: 80\% correct

696 performance (and at least $50 \%$ on both right and left trials) within any 30 -trial moving window.

697 Intradimensional Shift (IDS): A new pair of relevant stimuli replaced the stimuli used for SD and CD, while

698 the irrelevant stimuli remained unchanged. For animals initially trained on whisker stimuli, the new stimuli

699 were a $210 \mathrm{~Hz}$ sinusoid (lick-left) and a train of Poisson-distributed square-wave clicks averaging $210 \mathrm{~Hz}$

700 (lick-right). For animals initially trained on the odor rule, the new stimuli were almond oil (lick-left) and

701 orange extract (lick-right). This phase tests the animal's ability to learn a new stimulus-reward contingency

702 and discriminate between two new stimuli from the same task-relevant dimension. The criterion for passing

703 IDS was the same as for SD and CD.

704 Reversal (Rev): The same set of relevant and irrelevant stimuli were used as in IDS, but the left-right

705 mapping was reversed. This phase requires the animal to suppress and replace a previously learned

706 stimulus-response contingency. Criterion was the same as in SD, CD, and IDS.

707 Extradimensional Shift (EDS): The same whisker and odor stimuli were used as in IDS and Rev, but the 708 previously irrelevant modality now became the relevant modality and vice versa. Because relevant and 709 irrelevant stimuli were randomly and independently chosen on each trial, $50 \%$ of trials were congruent and

$71050 \%$ were incongruent. This phase requires the animal to associate a new stimulus category, the previously

711 irrelevant stimulus modality, with a left-right response mapping. Criterion was the same as in all preceding

712 task phases.

713 Intradimensional Shift 2 (IDS2): The stimuli in the newly relevant modality were replaced, while those in

714 the newly irrelevant modality were unchanged. This phase serves to ensure that the animal has learned to

715 associate left-right stimulus mappings with multiple exemplar sets in each modality, thereby establishing

716 an abstract attentional set within the newly relevant modality. Criterion was the same as in all preceding

717 task phases.

718 Serial Extradimensional Set-Shifting (SEDS): For each SEDS session, the relevant stimulus modality

719 was chosen randomly at the start of the session. Stimuli during these sessions were the same stimuli used

720 in the IDS2 phase (whisker: $210 \mathrm{~Hz}$ sinusoid, left, and Poisson click train, right; odor: almond oil, left, and

721 orange extract, right). Upon reaching criterion (still $80 \%$ correct and $50 \%$ on both left and right in a 30 -trial

722 moving window), the modality rule was automatically switched. This phase tests the animal's ability to

723 integrate trial feedback to flexibly switch between task rules. As with previous phases, relevant and

724 irrelevant stimuli were randomly and independently generated on each trial. To maximize the number of

725 set-shifts in each session, sessions continued until animals reached satiety (ten consecutive non-response

726 trials) or consumed $1.1 \mathrm{~mL}$ of water.

\section{Fixed tissue processing and imaging}

728 Animals were transcardially perfused with 4\% paraformaldehyde and 1x PBS. Heads were removed and

729 incubated with all tissues intact in $4 \%$ PFA at $6^{\circ} \mathrm{C}$. Brains were dissected after 24 hours and fixed in $4 \%$

730 PFA for an additional 24 hours before being dehydrated in a 30\% sucrose solution (24-72 hours, until

731 submerged). Tissue was sectioned coronally (in $45 \mu \mathrm{m}$ sections) at $-20^{\circ} \mathrm{C}$ and submerged in PBS until

732 mounting. (When not in the cryostat tissue was stored at $6^{\circ} \mathrm{C}$ ). Samples were mounted on $25 \times 75 \times 1 \mathrm{~mm}$

733 slides (Cole Parmer or Fisher Superfrost) with Sigma brand, DAPI-infused Fluoroshield mounting

734 medium. For the L5 vs L6 PFC-MDT dual labeling experiment, Fluoroshield without DAPI was used, to

735 enable BFP visualization. 
736 Imaging was carried out on the Leica DM 5500 B microscope with a Leica DFC360 FX fluorescent camera

737 (7x magnification) and EL600 light source, using a 10x apochromat objective with an NA of 0.4. Images

738 were taken in two channels - the red channel used a CY3 filter cube (excitation filter 545/40, dichroic mirror

739 565, emission filter 610/76) at a 90ms exposure. The blue channel was captured with a DAPI filter cube

740 (excitation 360/40, dichroic 400, emission 470/40) at a 100ms exposure. Both channels were imaged with

741 full laser power and no gain. To allow for comparison across brain regions, both within and across animals,

742 these imaging parameters were held constant for images gathered for the rabies tracing and fluorescent

743 axon tracing experiments.

744 Images were acquired using the Leica Acquisition System (LASX) (Version 3.6) navigator feature to capture 745 each sample as a series of 1281.71 x 957.36 um (1392x 1040 px) tiles, which were stitched (with no overlap 746 or blending) by the software during acquisition to produce the complete image.

\section{Rabies tracing}

748 Monosynaptic inputs to PFC-VMS and PFC-MDT projection neurons were labeled by using a cross749 sectional three-virus approach. At 4 weeks prior to sacking, AAV encoding the TVA rabies B19 glycoprotein 750 (pAAV-EF1a-FLEX-TVA/B19) was injected into PFC. Virus was infused using a $36 \mathrm{~g}$ beveled NanoFil 751 needle (WPI) and a 10 $\mu \mathrm{L}$ Hamilton syringe. Infusions of 250nL were made at each of four sites within PFC:

752 AP $+1.75 \mathrm{~mm}, \mathrm{ML}-0.2 \mathrm{~mm}$ and $-0.5 \mathrm{~mm}$, DV $-2.0 \mathrm{~mm}$ and $-1.5 \mathrm{~mm}$ (brain surface). Virus was infused at a rate 753 of $50 \mathrm{~nL} / \mathrm{min}$. In the same surgery, retrogradely transported rAAV2-CAG-Cre was injected into MDT (AP $7541.2 \mathrm{~mm}, \mathrm{ML}-0.35 \mathrm{~mm}$, DV $-3.2 \mathrm{~mm}$ ) or VMS (AP $+1.25 \mathrm{~mm}, \mathrm{ML}-1.25 \mathrm{~mm}$, DV $-4.7 \mathrm{~mm}$ ) in a volume of $500 \mathrm{~nL}$.

755 Following the dual infusions, Vetbond tissue adhesive was applied to the craniotomy over the exposed 756 dura, and the skin was sutured into place using 6-0 silk sutures. Three weeks later, a second infusion 757 surgery was performed to infuse EnvA G-Deleted Rabies-mCherry in PFC. Using a Nanoject nanoliter 758 injector and a borosilicate pulled glass micropipette, 50nL of virus was infused at either a cortically 759 superficial (ML $-0.3 \mathrm{~mm}$ ) or deep (ML -0.6mm) site (AP +1.75, DV -1.7). The animal was again sutured and 760 allowed to recover 1 week before sacking.

\section{Fluorescent axon tracing}

762 Surgical preparation, craniotomy, and infusion procedures were the same as those for GCaMP and 763 stGtACR2 projection labeling experiments. AAV1-CAG-DIO-tdTomato was infused in PFC at four sites: AP $764+1.75 \mathrm{~mm}$, ML $-0.2 \mathrm{~mm}$ and $-0.5 \mathrm{~mm}$, DV $-2.0 \mathrm{~mm}$ and $-1.5 \mathrm{~mm}$, at a volume of $250 \mathrm{~nL}$ per site and a rate of $76550 \mathrm{~nL} / \mathrm{min}$. rAAV2-CAG-Cre (500nL) was infused in MDT (AP $-1.2 \mathrm{~mm}, \mathrm{ML}-0.35 \mathrm{~mm}$, DV $-3.2 \mathrm{~mm}$ ) or VMS 766 (AP $+1.25 \mathrm{~mm}, \mathrm{ML}-1.25 \mathrm{~mm}$, DV $-4.7 \mathrm{~mm}$ ) in a volume of $500 \mathrm{~nL}$. Animals were sutured and allowed to 767 recover for four weeks before sacking.

\section{Image processing of rabies-labeled and fluorescent axon histology}

769 Coronal slices were mounted and imaged from $\sim A P+3 \mathrm{~mm}$ to $\sim A P-4 \mathrm{~mm}$, spanning the frontal pole of the neocortex to the ventral/caudal hippocampus and surrounding regions. Imaging was performed at

$7711 \mu \mathrm{m} /$ pixel resolution and tiled using Leica software. Image cropping, alignment, de-warping, registration to

772 the Allen Common Coordinate Framework (Wang et al., 2020), and annotation of cell locations was

773 performed using custom Matlab algorithms adapted in part from the cortex-lab/allenCCF package

774 (Shamash et al., 2018). Histological images were mapped to corresponding atlas sections by annotating

775 images with manual control points and generating piecewise regression image transformations (Matlab

776 fitgeotrans). Individual labeled neurons were then individually annotated in Matlab and mapped onto atlas

777 locations using the Allen CCF structure tree (data.cortexlab.net/allenCCF).

779 Electrophysiological recordings

780 stGtACR2 Photoactivation 
781 AAV1-hSyn-stGtACR2-FusionRed was infused in PFC (AP +1.75mm, ML -0.2mm / -0.5mm, DV -2.0mm / $782-1.5 \mathrm{~mm}, 250 \mathrm{~nL} / \mathrm{site})$. Animals were sutured and allowed to recover for 4 weeks. On the day of the recording 783 session, they were anesthetized under isoflurane (2\%) and transitioned to $0.5 \%$ isoflurane over a $20 \mathrm{~min}$

784 monitoring period. The craniotomy from the earlier viral transfusion was widened to an area of $\sim 2 \mathrm{~mm} x$ 785 2mm. A 32-channel silicon probe, coupled to a $200 \mu \mathrm{m}$-core, 0.6NA optical fiber (H6b-style optrode, 786 Cambridge Neurotech) was lowered into the brain at a rate of $\sim 100 \mu \mathrm{m} / \mathrm{min}$ to a depth of $1.7 \mathrm{~mm}$ ventral at 787 the tip. Recording were gathered using an Intan analog-to-digital acquisition system. Spikes were sampled 788 at $20 \mathrm{KHz}$ with a $1 \mathrm{~Hz}$ high-pass filter and $60 \mathrm{~Hz}$ notch filter, and recording channels were referenced against 789 an alligator clip secured to the neck muscle. Raw voltage recordings were later bandpass filtered between $790250 \mathrm{~Hz}$ and $20 \mathrm{KHz}$ using Matlab'd bandpass function, and multiunit spiking activity was thresholded at -

$7910.1 \mathrm{mV}$.

\section{Measuring muscimol-induced silencing}

793 Anesthesia was administered as described above in the photoactivation experiment. A larger ( 2mm ML $\mathrm{x}$ $794 \sim 3 \mathrm{~mm}$ AP) craniotomy was opened over frontal cortex. The animal's head was raised in order to provide a 795 flat (rather than sloped) cortical surface over frontal areas. A multielectrode array (Innovative 796 Neurophysiology Inc.) was arrayed in two rows of 8 , spaced at $150 \mu \mathrm{m}$ between columns and rows. The 797 rows were arranged along the AP axis, with the most anterior recording site at AP $+2.0 \mathrm{~mm}$, DV $-0.5 \mathrm{~mm}$. 798 and the two rows at $0.3 \mathrm{~mm}$ and $0.5 \mathrm{~mm}$ left of the midline. Muscimol $(1 \mu \mathrm{g} / \mu \mathrm{L})$ was infused at a rate of $79950 \mathrm{~nL} / \mathrm{min}$, for a total of $0.25 \mu \mathrm{L}$, through a $36 \mathrm{~g}$ needle positioned in between the most anterior pair of 800 electrodes and angled $10^{\circ}$ toward the anterior. Spontaneous activity during and following infusion was 801 recorded and processed with the same settings as in the photoactivation experiment described above.

\section{STATISTICAL ANALYSIS}

\section{Tensor component analysis (TCA)}

805 To estimate latent population activity associated with each task variable on a trial-to-trial basis, we used 806 an unsupervised tensor component analysis (TCA) approach (Williams et al., 2018) To avoid artifactual 807 sources of variance introduced by session registration, this analysis was performed independently for each 808 mouse and session. Trial-aligned $z$-scored activity traces (trial $\mathbf{x}$ neuron $\mathbf{x}$ time) were first normalized to a 809 nonnegative range and smoothed with a gaussian kernel. They were then decomposed into a set of related 810 trial, neuron, and temporal factors. We evaluated models using both standard TCA and nonnegative TCA, 811 in which weights are constrained to positive values, ultimately selecting nonnegative TCA as it provided a 812 more consistent fit. The trial components of these factors were then regressed onto the five primary task 813 variables (whisker, odor, response, outcome, and rule) using Matlab's glmfit function. Factors that were 814 significantly modulated (Bonferroni corrected t-test) by a given variable in the trial domain were then pooled 815 to estimate temporal profiles for latent population activity underlying each task variable. Tensor 816 reconstruction was performed by performing matrix multiplication on the component neuron*factor, 817 trial ${ }^{\star}$ factor, and time* factor matrices. Temporal components shown in Figure $2 \mathrm{G}$ are from factors found to 818 be associated with each task variable with a p-value $<10 \mathrm{e}^{10}$ using the GLM. This stringent criterion was 819 used in order to include only the top $5 \%$ of most reliably modulated factors. Less stringent criteria resulted 820 in the inclusion of factors with a high variance among temporal components.

\section{Linear decoding with SVM maximum margin classifiers}

823 Population decoding was performed with maximum-margin linear decoders (Matlab fitcsvm). At each 824 iteration data were separated into equally sized training and testing sets, and in each set, trials were 825 separated into equal numbers from each binary value for the feature of interest. To combine inputs from 826 neurons across multiple sessions, and to remove inter-trial variability each of these trial groups was than 827 randomly separated into 16 trial groups, each of which was averaged into one super-trial to serve as a 828 decoder input (16 super-trials for one value of the feature of interest and 16 from the opposing value). This 
829 number was calibrated to ensure robust trial subsampling within training and testing sets while removing

\section{Principal components analysis (PCA)}

835 PCA was used to identify the trial-aligned activity patterns responsible for the greatest variance within the

\section{Supplemental Figure Titles and Legends}

\section{Figure S1. Muscimol infusion experiment}

864. 16-channel multi-electrode array (Innovative Neurophysiology Inc.) used to measure the spatial extent of activity space of the neural traces (Fig. 5J). PCA was performed using Matlab's native pca function, and the first three resulting components were plotted through trial-aligned time. PCA was performed separately on trial-averaged traces from PFC-VMS and PFC-MDT sets (neuron $\mathrm{x}$ time).

\section{General Linear Model}

Matlab's fitglm function was used to fit a GLM model to projection cell type and topological location (depth from pial surface). Fitglm was also used to model multiple single neuron activity as a function of 7 task variables: whisker stimulus, odor stimulus, response direction (current trial), response direction (previous trial), trial outcome (current trial), trial outcome (previous trial), and task rule. Unless otherwise specified, GLMs were modeled with normal distributions, as linear functions (no interaction effects) with y-intercept terms, and with identity linkages. For analysis of individual neuron responses to task variables, a separate GLM was computed performed for each time point in the trial independently.

$$
\hat{Y}=\beta_{0}+\beta_{1} p+\beta_{2} d+\beta_{3} p \cdot d
$$

\section{Euclidean distance and vector angles in neural activity space}

of the differences between the two conditions across all neurons in the set.

Euclidean distance was also computed on activity vectors scaled by the vector weights of their corresponding SVM decoders in order to scale the amplitude of feature-selective cells relative to noise. Vector angles were computed as the dot product of the difference vectors, divided by the product of their norms. This value was converted into an angle of degrees by taking its inverse cosine.

$$
\theta_{x y}=\cos ^{-1}(x \cdot y) \div(\|x\| \cdot\|y\|)
$$
muscimol silencing effects. Photograph taken through a binocular surgical stereoscope (Kopf). The diagonal needle in the upper left is the infusion needle ( $36 \mathrm{~g}$ blunt, NanoFil), tilted to an anterior angle of 
Spellman et al., 2020

$86710^{\circ}$ off vertical. The needle was positioned between the anterior-most pair of electrodes. Electrode rows

868 were spaced at $150 \mu \mathrm{m}$ apart, $150 \mu \mathrm{m}$ spacing within rows.

$8 \circledast$. Histological sections from a fluorescent muscimol injection at three horizontal planes. Red: Muscimol-

870 TMR-X (Sigma Aldrich); Blue: DAPI.

87 . Comparison of overt licking behavior in animals receiving muscimol, saline, and no injection. Rank sum

872 for the BL-MUS comparison: $p=0.88$.

8ד3. Histological comparison from a fluorescent muscimol injection at five coronal planes. Red: Muscimol-

874 TMR-X (Sigma Aldrich); Blue: DAPI.

875

\section{Figure S2. Sagittal section from prism-implant}

877 hSyn-GCaMP6f or hSyn-DIO-GCaMP6f was injected at A/P $+1.75 \mathrm{~mm}, \mathrm{M} / \mathrm{L}-0.2 \mathrm{~mm}$ and $-0.5 \mathrm{~mm}, \mathrm{D} / \mathrm{V}-$

$8782.0 \mathrm{~mm}$ and $-1.5 \mathrm{~mm}$. An atlas image from $\mathrm{M} / \mathrm{L}-0.42 \mathrm{~mm}$ was superimposed, with the corpus callosum (cc)

879 used to co-register the atlas with the histological image. PL: prelimbic cortex; IL: infralimbic cortex;

$880 \mathrm{ACC}(\mathrm{rd})$ : rostro-dorsal anterior cingulate

\section{Figure S3. Example Source Localization with CNMF-E, PFC-MDT}

88\&. Left: Session-wide activity projection for an animal labeled with GCaMP6f in PFC-MDT projection

884 neurons. The value for each pixel corresponds with the pixel's standard deviation across all frames of

885 imaging, normalized to its mean value (its session z-score). Center: Putative cell footprints extracted from

886 the recording using CNMF-E. Grey contours are boundaries of spatial components, red dots are median

887 centroids ( $N=501$ putative cells, 15437 frames, $0.289 \mathrm{f} / \mathrm{s}$, galvano scanning). iii: First three PCA scores

888 for each pixel in a represenative putative cell, its nearest neighbor, and neuropil, defined as an equal or

889 greater number of pixels drawn from immediately outside the footprint and not within any other footprint.

890 iv: Population cumulative distributions of isolation distances for the population. Red: isolation from

891 nearest neighbor. Gray: isolation from neuropil. Blue: signal/noise ratios for pixels from identified

892 neurons.

89. Same as (A), for a second PFC-MDT animal.

894

895 Figure S4. Example Source Localization with CNMF-E, PFC-VMS

896 Same as Fig. S3 for two PFC-VMS animals.

897

898 Figure S5. Example Signal Extraction for a PFC-MDT animal

89A. Example signals, PFC-MDT, same animal as (S3-A). i) Ten representative raw fluorescence traces from

900 putative neurons identified in two successive SEDS sessions. ii) (top): Individual video frames from a

901 single calcium transient event. Frames shown are downsampled to a rate of $1 / 2$ the recording rate.

902 Bottom: traces from the corresponding neuron as raw fluorescence (black, units are pixel brightness as

903 unsigned 16-bit integers, maximum value 65,536), denoised $\Delta \mathrm{f} / \mathrm{f}$ (red), and deconvolved instantaneous

904 spike pobability (blue).

9(B. As in (A), for a second PFC-MDT animal (same as S3-B).

906

\section{Figure S6. Additional examples of signal extraction}

908 As in S5. Same animals as in S4. 
910 Figure S7. Example cross-session cell footprint alignment using the CellReg cell registration

911 algorithm

91A. CNMF-E-extracted cell footprints from session 1 and 2, same animal as in S3-A and S5-A. Green-filled

913 footprints are those detected across all recording sessions.

918. Pre-aligned (left) and post-aligned (right) cell footprints. Non-rigid alignment method.

916. Pre-aligned (left) and post-aligned (right) cell centroids.

910. Post-alignment offset of putative matching cell footprints.

91日. Centroid distance histograms for putative matching (green) and non-matching (red) pairs of footprints.

918

919 Figure S8-S10. Additional examples of cross-session cell footprint alignments

920 Animals from S3-B, S4-A, and S4-B, respectively.

922 Figure S11. Task modulation of nonnegative TCA Factors

92A. Representative factor estimates identified using nonnegative TCA as defined by neuron, trial, and time

924 components. Data shown is from a single mouse and session (186 neurons x 566 trials $\times 54$ timepoints)

925 decomposed into 10 factors. Neuron components are sorted for each factor independently.

926. Trial components for each factor were then regressed using a GLM onto task variables. Asterisks denote

927 significance (t-statistic, $\mathrm{p}<0.05)$.

\section{Figure S12. Temporal dynamics of response direction and trial outcome representation}

930 Two additional examples of neurons individually representing response direction and trial outcome, 931 accompanying the examples shown in Fig. 3A.

93A. Left: $z$-scored activity on 1071 individual trials (y-axis), and mean z-scored activity (top) over the pre-trial, 933 trial, and post-trial ITI epochs (x-axis) for trials on which the animal responded left (cyan) and for which

934 the animal responded right (magenta). Right: GLM estimate of trial-specific activity.

935. Left: activity rastors (y-axis) and mean activity (top) for a second neuron, same session. Cyan: incorrect 936 trials; magenta: correct trials. Right: GLM estimate of trial-specific activity.

938 Figure S13. Fluorescence in long-range axons from PFC-VMS and PFC-MDT projection neurons

934. Long-range efferent axons from PFC-VMS neurons. Red: CAG-DIO-tdTomato; blue: DAPI. i) Fluorescent 940 axons in the nucleus accumbens (NAcc), substantia innominata (SI), endopiriform nucleus (EP), and 941 olfactory tubercle (OT). ii) Axon collaterals from PFC-VMS neurons in the claustrum (CL) and agranular 942 insular cortex (AIA). iii) Axon collaterals from PFC-VMS neurons in basolateral amygdala (BLA).

94B. Fluorescent axons from PFC-MDT neurons in mediodorsal thalamus (MDT), zona incerta (ZI), and the 944 ventral anterolateral thalamic nucleus (VALT).

940. Coronal slices at multiple A/P cross-sections from within the same PFC-VMS labeled animal. i) Nucleus 946 accumbens and surrounding regions at $+1.4 \mathrm{~mm},+1.25 \mathrm{~mm}$, and $+1.1 \mathrm{~mm}$ relative to bregma (Allen CCF).

947 ii) Medial thalamus and surrounding regions in the same animal, same laser and confocal microscope 948 settings. 
949. Coronal slices at multiple A/P cross-sections from within the same PFC-MDT labeled animal. i) NAcc and 950 surrounding regions at $+1.4 \mathrm{~mm},+1.25 \mathrm{~mm}$, and $+1.1 \mathrm{~mm}$ relative to bregma. ii). Medial thalamus and 951 surrounding regions in the same animal.

9ङ2. Contour plots of axon fluorescence density from a composite of 5 PFC-VMS and 5 PFC-MDT labeled 953 animals. Horizontal cross-section at $4.1 \mathrm{~mm}$ ventral to bregma (Allen CCF). Concentric contours

954 correspond with pixel brightness, which is represented as a percentile from each animal's pixel value 955 distribution. Outermost of five concentric contours corresponds with the $98^{\text {th }}$ percentile, and the innermost 956 enclosed contour corresponding with the $100^{\text {th }}$ percentile for pixel brightness.

957. Left: Example coronal cross-section of medial thalamus and surrounding regions from a PFC(L6)-MDT 958 labeled animal (Cav-Cre in MDT and Flex-tdTomato in PFC). right: Composite contour plots for 5 959 PFC(L5)-MDT (green) and 2 PFC(L6)-MDT (black) labeled animals. Pixel brightness scale same as (E).

\section{Figure S14. Rabies retrograde labeling experiment}

964. Schematic diagram of G-deleted rabies tracing experiment. At 4 weeks prior to sacking, an AAV encoding 963 the TVA rabies B19 glycoprotein (pAAV-EF1a-FLEX-TVA/B19) was injected into PFC, and in the same 964 surgery, retrogradely transported rAAV2-CAG-Cre was injected into MDT or VMS (see Methods). at 1 965 week prior to sacking, animals underwent an injection of EnvA G-Deleted Rabies-mCherry into either 966 superficial (300um from midline) or deep (600um from midline) PFC, at a volume of 50nL). This approach 967 allows for monosynaptic retrograde labeling of projection neurons.

9\$. Example histological images of EnvA G-Deleted Rabies-mCherry labeling. i: labeling around the injection 969 site. Injections were targeted to the PL/IL border. ii: labeling in caudo-ventral ACC. This region showed 970 enhanced labeling in deep-injected animals relative to superficially-injected animals. iii: labeling in the 971 ventral antero-lateral nucleus of the thalamus, one of numerous thalamic nuclei to show robust labeling, 972 including medio-dorsal thalamus, retrospenial nucleus, paraventricular thalamus, nucleis of reuniens, and 973 parataenial nucleus. iv: labeling in the diagonal band nucleus. Robust labeling was seen throughout the

974 cholinergic nuclei of the basal forebrain, including the medial septum, the preoptic areas, and substantia 975 innominata.

976. Relative cell densities in deep vs superficially-injected animals across brain areas. Regions shown are 977 the 50 most cell-rich regions.

978. Support-vector-machine-based decoders for within-region classification of cells from the two PFC 979 injection depths. Inputs are the $\mathrm{x}, \mathrm{y}$, and $\mathrm{z}$ coordinates of each neuron, and each classifier was tested 980 using 20-fold cross-validation and assuming uniform between-class distribution. Left: optimal support 981 vector for ventral agranular insular cortex, which did not show a difference in total cell number between 982 injection types, but in which a rostro-dorsal / caudo-ventral boundary between cell types is observed. 983 Right: decoder performance, as well as performance on shuffled data, for each of the regions shown in 984 C. Error bars: standard deviations. Asterisks: 2-sided t-test, $\mathrm{p}<0.05$.

985

\section{Figure S15. Comparison of response and outcome signals by temporal trial alignment}

984. Mean +/- SEM for trial-aligned GLM coefficients of neurons significantly modulated by trial outcome 988 direction. Modulated neurons include those with significance at any timepoint (Bonferroni-corrected for 989 the 43 trial timepoints). Blue: coefficients aligned to stimulus offset; black: coefficients aligned to first lick 990 within the response window.

998. Same as (A), for response direction. Red: coefficients aligned to stimulus offset; black: coefficients 992 aligned to first lick within the response window. 
Spellman et al., 2020

990. Cumulative histogram of times of peak absolute activity (relative to the mean over the 43 trial timepoints)

994 for each cell. Blue: peaks aligned to stimulus offset; red: peaks aligned to first lick within response

995 window.

997 Table S16. Summary of behavioral statistics for optogenetic experiment

\section{References}

1002 Adesnik, H., and Naka, A. (2018). Cracking the Function of Layers in the Sensory Cortex. Neuron 1003 VOLUME 100, ISSUE 5, P1028-1043.

1004 Akrami, A., Kopec, C., Diamond, M., and Brody, C. (2018). Posterior parietal cortex represents sensory 1005 history and mediates its effects on behaviour. Nature Feb 15;554(7692):368-372.

1006 Andermann, M., Gilfoy, N., Goldey, G., Sachdev, R., Wölfel, M., McCormick, D., Reid, R., and Levene, M. 1007 (2013). Chronic Cellular Imaging of Entire Cortical Columns in Awake Mice Using Microprisms. Neuron 1008 80, 900-913.

1009 Aoki, S., Liu, A.W., Zucca, A., Zucca, S., and Wickens, J.R. (2015). Role of Striatal Cholinergic 1010 Interneurons in Set-Shifting in the Rat. J. Neurosci. 35, 9424-9431.

1011 Bari, B., Grossman, C., Lubin, E., Rajagopalan, A., Cressy, J., and Cohen, J. (2019). Stable

1012 Representations of Decision Variables for Flexible Behavior. Neuron 103, 922-933.

1013 Bernacchia, A., Seo, H., Lee, D., and Wang, X. (2011). A reservoir of time constants for memory traces in 1014 cortical neurons. Nat Neurosci. Mar;14(3):366-72.

1015 Biró, S., Lasztóczi, B., and Klausberger, T. (2019). A Visual Two-Choice Rule-Switch Task for Head1016 Fixed Mice. Front Behav. Neurosci. 06 June.

1017 Birrell, J., and Brown, V. (2000). Medial frontal cortex mediates perceptual attentional set shifting in the 1018 rat. J. Neurosci. 20, 4320-4324.

1019 Bissonette, G., and Roesch, M. (2015). Neural correlates of rules and conflict in medial prefrontal cortex 1020 during decision and feedback epochs. Front Behav. Neurosci. 9: 266.

1021 Bissonette, G., Martins, G., Franz, T., Harper, E., Schoenbaum, G., and Powell, E. (2008). Double 1022 dissociation of the effects of medial and orbital prefrontal lesions on attentional and affective shifts in 1023 mice. J. Neurosci. 28, 11124-11130.

1024 Bissonette, G., Powell, E., and Roesch, M. (2013). Neural structures underlying set-shifting: roles of 1025 medial prefrontal cortex and anterior cingulate cortex. Behav. Brain Res. 250, 91-101.

1026 Block, A., Dhanji, H., Thompson-Tardif, S., and Floresco, S. (2007). Thalamic-prefrontal cortical-ventral 1027 striatal circuitry mediates dissociable components of strategy set shifting. Cereb. Cortex 17.

1028 Bortolato, B., Miskowiak, K., Köhler, C., Maes, M., Fernandez, B., Berk, M., and Carvalho, A. (2016). 1029 Cognitive remission: a novel objective for the treatment of major depression? BMC Med. 14, Article 1030 number: 9. 
1031 Brigman, J., Bussey, T., Saksida, L., and Rothblat, L. (2005). Discrimination of multidimensional visual 1032 stimuli by mice: intra- and extradimensional set shifts. Behav. Neurosci. 119, 839-842.

1033 Ceaser, A., Goldberg, T., Egan, M., McMahon, R., Weinberger, D., and Gold, J. (2008). Set-shifting 1034 ability and schizophrenia: a marker of clinical illness or an intermediate phenotype? Biol Psychiatry 64, $1035782-788$.

1036 Chen, T., Wardill, T., Sun, Y., Pulver, S., Renninger, S., Baohan, A., Schreiter, E., Kerr, R., Orger, M., 1037 Jayaraman, V., et al. (2013). Ultrasensitive fluorescent proteins for imaging neuronal activity. Nature ul 1038 18;499(7458):295-300.

1039 Christianini, N., and Shawe-Taylor, J. (2000). An Introduction to Support Vector Machines and Other 1040 Kernel-Based Learning Methods (Cambridge, UK: Cambridge University Press).

1041 Collins, D., Anastasiades, P., Marlin, J., and Carter, A. (2018). Reciprocal Circuits Linking the Prefrontal 1042 Cortex with Dorsal and Ventral Thalamic Nuclei. Neuron 98, 366-379.

1043 Corbetta, M., and Shulman, G. (2002). Control of goal-directed and stimulus-driven attention in the brain. 1044 Nat Rev Neurosci Mar;3(3):201-15.

1045 Crowe, S., and Ellis-Davies, G. (2014). Longitudinal in vivo two-photon fluorescence imaging. J. Comp. 1046 Neurol. 522:1708-1727.

1047 Denk, W., Strickler, J., and Webb, W. (1990). Two-Photon Laser Scanning Fluorescence Microscopy. 1048 Science Vol. 248, No. 4951., 73-76.

1049 Desimone, R., and Duncan, J. (1995). Neural mechanisms of selective visual attention. Annu Rev 1050 Neurosci 18:193-222.

1051 Disner, S.G., Beevers, C.G., Haigh, E.A.P., and Beck, A.T. (2011). Neural mechanisms of the cognitive 1052 model of depression. Nat. Rev. Neurosci. 12, 467-477.

1053 Durstewitz, D., Vittoz, N., Floresco, S., and Seamans, J. (2010). Abrupt transitions between prefrontal 1054 neural ensemble states accompany behavioral transitions during rule learning. Neuron 66, 438-448.

1055 Ellwood, I., Patel, T., Wadia, V., Lee, A., Liptak, A., Bender, K., and Sohal, V. (2017). Tonic or Phasic 1056 Stimulation of Dopaminergic Projections to Prefrontal Cortex Causes Mice to Maintain or Deviate from 1057 Previously Learned Behavioral Strategies. J Neurosci 37(35): 8315-8329.

1058 Floresco, S., Ghods-Sharifi, S., Vexelman, C., and O, M. (2006). Dissociable Roles for the Nucleus 1059 Accumbens Core and Shell in Regulating Set Shifting. J. Neurosci. 26, 2449-2457.

1060 Fox, M., Barense, M., and Baxter, M. (2003). Perceptual attentional set-shifting is impaired in rats with 1061 neurotoxic lesions of posterior parietal cortex. J Neurosci Jan 15;23(2):676-81.

1062 Friedrich, J., Zhou, P., and Paninski, L. (2017). Fast online deconvolution of calcium imaging data. PLoS 1063 Comput. Biol. https://doi.org/10.1371/journal.pcbi.1005423.

1064 Garrison, J., Erdeniz, B., and Done, J. (2013). Prediction error in reinforcement learning: a meta-analysis 1065 of neuroimaging studies. Neurosci Biobehav Rev Aug;37(7):1297-310.

1066 Gonda, X., Pompili, M., Serafini, G., Carvalho, A.F., Rihmer, Z., and Dome, P. (2015). The role of 1067 cognitive dysfunction in the symptoms and remission from depression. Ann. Gen. Psychiatry 14, 27. 
1068 Halleland, H., Haavik, J., and Lundervold, A. (2012). Set-shifting in adults with ADHD. J Int Neuropsychol 1069 Soc 18, 728-737.

1070 Harris, K., and Shepherd, G. (2015). The neocortical circuit: themes and variations. Nat. Neurosci. 18, 1071 pages170-181.

1072 Harvey, P., Green, M., Keefe, R., and Velligan, D. (2004). Cognitive Functioning in Schizophrenia: A 1073 Consensus Statement on Its Role in the Definition and Evaluation of Effective Treatments for the Illness.

1074 J. Clin. Psychiatry 65, 361-372.

1075 Hauser, T., lannaccone, R., Staempfli, P., and Drechsler, R. (2013). The Feedback-Related Negativity 1076 (FRN) revisited: New insights into the localization, meaning and network organization. Neurolmage 84.

1077 Heisler, J.M., Morales, J., Donegan, J.J., Jett, J.D., Redus, L., and O'Connor, J.C. (2015). The Attentional 1078 Set Shifting Task: A Measure of Cognitive Flexibility in Mice. J. Vis. Exp. JoVE.

1079 Hill, S.K., Bishop, J.R., Palumbo, D., and Sweeney, J.A. (2010). Effect of second-generation 1080 antipsychotics on cognition: current issues and future challenges. Expert Rev. Neurother. 10, 43-57.

1081 Hyman, J., Holroyd, C., and Seamans, J. (2017). A Novel Neural Prediction Error Found in Anterior 1082 Cingulate Cortex Ensembles. Neuron Jul 19;95(2):447-456.e3.

1083 Hymana, J., Maa, L., Balaguer-Ballesterb, E., Durstewitzb, D., and Seamansa, J. (2012). Contextual 1084 encoding by ensembles of medial prefrontal cortex neurons. PNAS 109, 5086-5091.

1085 Jazbec, S., Pantelis, C., Robbins, T., Weickert, T., Weinberger, D., and Goldberg, T. (2007). Intra1086 dimensional/extra-dimensional set-shifting performance in schizophrenia: Impact of distractors.

1087 Schizophr. Res. 89, 339-349.

1088 Kato, S., Fukabori, R., Nishizawa, K., Okada, K., Yoshioka, N., Sugawara, M., Maejima, Y., Shimomura, 1089 K., Okamoto, M., Eifuku, S., et al. (2018). Action Selection and Flexible Switching Controlled by the 1090 Intralaminar Thalamic Neurons. Cell Rep. 27;22(9):2370-2382.

1091 Kim, C., Cilles, S.E., Johnson, N.F., and Gold, B.T. (2012). Domain General and Domain Preferential 1092 Brain Regions Associated with Different Types of Task Switching: A Meta-Analysis. Hum. Brain Mapp.

1093 33, 130-142.

1094 Liu, X., and Carter, A. (2018). Ventral Hippocampal Inputs Preferentially Drive Corticocortical Neurons in 1095 the Infralimbic Prefrontal Cortex. J Neurosci Aug 15;38(33):7351-7363.

1096 Ljubojevic, V., Luu, P., and De Rosa, E. (2014). Cholinergic Contributions to Supramodal Attentional 1097 Processes in Rats. J. Neurosci. 34(6):2264-2275.

1098 Low, R.J., Gu, Y., and Tank, D.W. (2014). Cellular resolution optical access to brain regions in fissures: 1099 Imaging medial prefrontal cortex and grid cells in entorhinal cortex. Proc. Natl. Acad. Sci. 111, 18739110018744.

1101 Lui, J., Nguyen, N., Grutzner, S., Darmanis, S., Peixoto, D., Wagner, M., Allen, W., Kebschull, J., 1102 Richman, E., Ren, J., et al. (2020). Differential encoding in prefrontal cortex projection neuron classes 1103 across cognitive tasks. Cell December 17, 2020DOI:https://doi.org/10.1016/j.cell.2020.11.046.

1104 MacDonald, A. 3rd, Cohen, J., Stenger, V., and Carter, C. (2000). Dissociating the role of the dorsolateral 1105 prefrontal and anterior cingulate cortex in cognitive control. Science. 
1106 Mahn, M., Gibor, L., Patil, P., Malina, K., Oring, S., Printz, Y., Levy, R., Lampl, I., and Yizhar, O. (2018). High-efficiency optogenetic silencing with somatargeted anion-conducting channelrhodopsins. Nat. Commun. 9:4125.

Mante, V., Sussillo, D., Shenoy, K., and Newsome, W. (2013). Context-dependent computation by recurrent dynamics in prefrontal cortex. Nature 7;503(7474):78-84.

1111 Marshel, J., KIm, Y., Machado, T., Quirin, S., Benson, B., Kadmon, J., Raja, C., Chibukhchyan, A.,

1112 Ramakrishnan, C., Inoue, M., et al. (2019). Cortical layer-specific critical dynamics triggering perception.

1113 Science Aug 9;365(6453):eaaw5202.

1114 Marton, T., Seifikar, H., Luongo, F., Lee, A., and Sohal, V. (2018). Roles of Prefrontal Cortex and

1115 Mediodorsal Thalamus in Task Engagement and Behavioral Flexibility. J Neurosci 38(10):2569-2578.

1116 Meyers, E., Freedman, D., Kreiman, G., Miller, E., and Poggio, T. (2008). Dynamic population coding of 1117 category information in inferior temporal and prefrontal cortex. J Neurophysiol 100, 1407-1419.

1118 Miller, P. (2016). Dynamical systems, attractors, and neural circuits. F1000Research 5.

1119 Miller, E., and Cohen, J. (2001). An integrative theory of prefrontal cortex function. Annu Rev Neurosci 1120 24:167-202.

1121 Milner, B. (1963). Effects of different brain lesions on card sorting: The role of the frontal lobes. Arch.

1122 Neurol. 9, 100-110.

1123 Murphy, F.C., Michael, A., and Sahakian, B.J. (2012). Emotion modulates cognitive flexibility in patients 1124 with major depression. Psychol. Med. 42, 1373-1382.

1125 Nakai, J., Ohkura, M., and Imoto, K. (2001). A high signal-to-noise Ca2+ probe composed of a single 1126 green fluorescent protein. Nat. Biotechnol. 19;137-141.

1127 Nakayama, H., Ibañez-Tallon, I., and Heintz, N. (2018). Cell-type specific contributions of medial 1128 prefrontal neurons to flexible behaviors. J Neurosci. 10.1523/JNEUROSCI.3537-17.2018.

1129 Otis, J.M., Namboodiri, V.M.K., Matan, A.M., Voets, E.S., Mohorn, E.P., Kosyk, O., McHenry, J.A., 1130 Robinson, J.E., Resendez, S.L., Rossi, M.A., et al. (2017). Prefrontal cortex output circuits guide reward 1131 seeking through divergent cue encoding. Nature 543, 103-107.

1132 Pnevmatikakis, E., and Giovannucci, A. (2017). NoRMCorre: An online algorithm for piecewise rigid 1133 motion correction of calcium imaging data. J. Neurosci. Methods Volume 291, Pages 83-94.

1134 Pnevmatikakis, E.A., Soudry, D., Gao, Y., Machado, T.A., Merel, J., Pfau, D., Reardon, T., Mu, Y., 1135 Lacefield, C., Yang, W., et al. (2016). Simultaneous Denoising, Deconvolution, and Demixing of Calcium 1136 Imaging Data. Neuron 89, 285-299.

1137 Prado, V., Janickova, H., Al-Onaizi, M., and Prado, M. (2017). Cholinergic circuits in cognitive flexibility. 1138 Neuroscience Mar 14;345:130-141.

1139 Proulx, E., Piva, M., Tian, M., Bailey, C., and Lambe, E. (2014). Nicotinic acetylcholine receptors in 1140 attention circuitry: the role of layer VI neurons of prefrontal cortex. Cell Mol Life Sci 71(7): 1225-1244.

1141 Rich, E., and Shapiro, M. (2007). Prelimbic/Infralimbic Inactivation Impairs Memory for Multiple Task 1142 Switches, But Not Flexible Selection of Familiar Tasks. J Neurosci Apr 25; 27(17): 4747-4755. 
1143 Rich, E., and Shapiro, M. (2009). Rat prefrontal cortical neurons selectively code strategy switches. J.

1144 Neurosci. 29, 7208-7219.

1145 Rigotti, M., Barak, O., Warden, M., Wang, X., Daw, N., Miller, E., and Fusi, S. (2013). The importance of mixed selectivity in complex cognitive tasks. Nature 497, 585-590.

1147 Rodgers, C., and DeWeese, M. (2014). Neural Correlates of Task Switching in Prefrontal Cortex and 1148 Primary Auditory Cortex in a Novel Stimulus Selection Task for Rodents. Neuron 82, 1157-1170.

1149 Sayyah, M., Eslami, K., AlaiShehni, S., and Kouti, L. (2016). Cognitive Function before and during 1150 Treatment with Selective Serotonin Reuptake Inhibitors in Patients with Depression or Obsessive1151 Compulsive Disorder. Psychiatry J. 2016.

1152 Schmitt, L., Wimmer, R., Nakajima, M., Happ, M., MofakHam, S., and Halassa, M. (2017). Thalamic 1153 amplification of cortical connectivity sustains attentional control. Nature May 11;545(7653):219-223.

1154 Schmitzer-Torbert, N., Jackson, J., Henze, D., Harris, K., and Redish, A. (2005). Quantitative measures 1155 of cluster quality for use in extracellular recordings. Neuroscience 131, 1-11.

1156 Senzai, Y., Fernandez-Ruiz, A., and Buzsaki, G. (2019). Layer-Specific Physiological Features and 1157 Interlaminar Interactions in the Primary Visual Cortex of the Mouse. Neuron VOLUME 101, ISSUE 3, 1158 P500-513.E5.

1159 Shamash, P., Carandini, M., Harris, K., and Steinmetz, N. (2018). A tool for analyzing electrode tracks 1160 from slice histology. BioRxiv doi: https://doi.org/10.1101/447995.

1161 Sharif, F., Tayebi, B., Buzsaki, G., Royer, S., and Fernandez-Ruiz, A. (2020). Subcircuits of Deep and 1162 Superficial CA1 Place Cells Support Efficient Spatial Coding across Heterogeneous Environments. 1163 Neuron Nov 14;S0896-6273(20)30858-8.

1164 Sheintuch, L., Rubin, A., Brande-Eilat, N., Geva, N., Sadeh, N., Pinchasof, O., and Ziv, Y. (2017). 1165 Tracking the Same Neurons across Multiple Days in Ca2+ Imaging Data. Cell Rep. 21, 1102-1115.

1166 Siniscalchi, M., Phoumthipphavong, V., Ali, F., Lozano, M., and Kwan, A. (2016). Fast and slow 1167 transitions in frontal ensemble activity during flexible sensorimotor behavior. Nat. Neurosci.

1168 Sep;19(9):1234-42.

1169 Siniscalchi, M., Wang, H., and Kwan, A. (2019). Enhanced Population Coding for Rewarded Choices in 1170 the Medial Frontal Cortex of the Mouse. Cereb Cortex Sep 13;29(10):4090-4106.

1171 Smith, M., and Kohn, A. (2008). Spatial and Temporal Scales of Neuronal Correlation in Primary Visual 1172 Cortex. J Neurosci 28(48): 12591-12603.

1173 Smout, C., Tang, M., Garrido, M., and Mattingley, J. (2019). Attention promotes the neural encoding of 1174 prediction errors. PLoS Biol 17(2): e2006812.

1175 Soudais, C., Laplace-Builhe, C., Kissa, K., and Kremer, E. (2001). Preferential transduction of neurons by 1176 canine adenovirus vectors and their efficient retrograde transport in vivo. FASEB J 15:2283-2285.

1177 Starkweather, C., Gershman, S., and Uchida, N. (2018). The Medial Prefrontal Cortex Shapes Dopamine 1178 Reward Prediction Errors under State Uncertainty. Neuron May 2;98(3):616-629.e6. 
1179 Stringer, C., and Pachitariu, M. (2019). Computational processing of neural recordings from calcium 1180 imaging data. Curr Opin Neurobiol 55, 22-31.

1181 Sul, J., Kim, H., Huh, N., Lee, D., and Jung, M. (2010). Distinct roles of rodent orbitofrontal and medial 1182 prefrontal cortex in decision making. Neuron May 13; 66(3): 449-460.

1183 Tait, D.S., Chase, E.A., and Brown, V.J. (2014). Attentional set-shifting in rodents: a review of 1184 behavioural methods and pharmacological results. Curr. Pharm. Des. 20, 5046-5059.

1185 Tervo, D., Hwang, B., Viswanathan, S., Gaj, T., Lavzin, M., Ritola, K., Lindo, S., Michael, S., Kuleshova, 1186 E., Ojala, D., et al. (2016). A Designer AAV Variant Permits Efficient Retrograde Access to Projection 1187 Neurons. Neuron Oct 6. pii: S0896-6273(16)30580-3.

1188 Toyomaki, A., Hashimoto, N., Kako, Y., Murohashi, H., and Kusumi, I. (2017). Neural responses to 1189 feedback information produced by self-generated or other-generated decision-making and their 1190 impairment in schizophrenia. PLoS ONE 2(8): e0183792.

1191 Turner, J., Parrish, J., Hughes, L., Toth, L., and Caspary, D. (2005). Hearing in Laboratory Animals: 1192 Strain Differences and Nonauditory Effects of Noise. Comp Med Feb; 55(1): 12-23.

1193 Wang, Q., Ding, S., Li, Y., Royall, J., Feng, D., Lesnar, P., Graddis, N., Naeemi, M., Facer, B., Ho, A., et 1194 al. (2020). The Allen Mouse Brain Common Coordinate Framework: A 3D Reference Atlas. Cell May 1195 14;181(4):936-953.e20.

1196 Williams, A., Kim, T., Wang, F., Vyas, S., Ryu, S., and Shenoy, K. (2018). Unsupervised Discovery of 1197 Demixed, LowDimensional Neural Dynamics across Multiple Timescales through Tensor Component 1198 Analysis. Neuron 98, 1-17.

1199 Wimmer, R., Schmidt, L., Davidson, T., Nakajima, M., Deisseroth, K., and Halassa, M. (2015). Thalamic 1200 control of sensory selection in divided attention. Nature Oct 29;526(7575):705-9. 

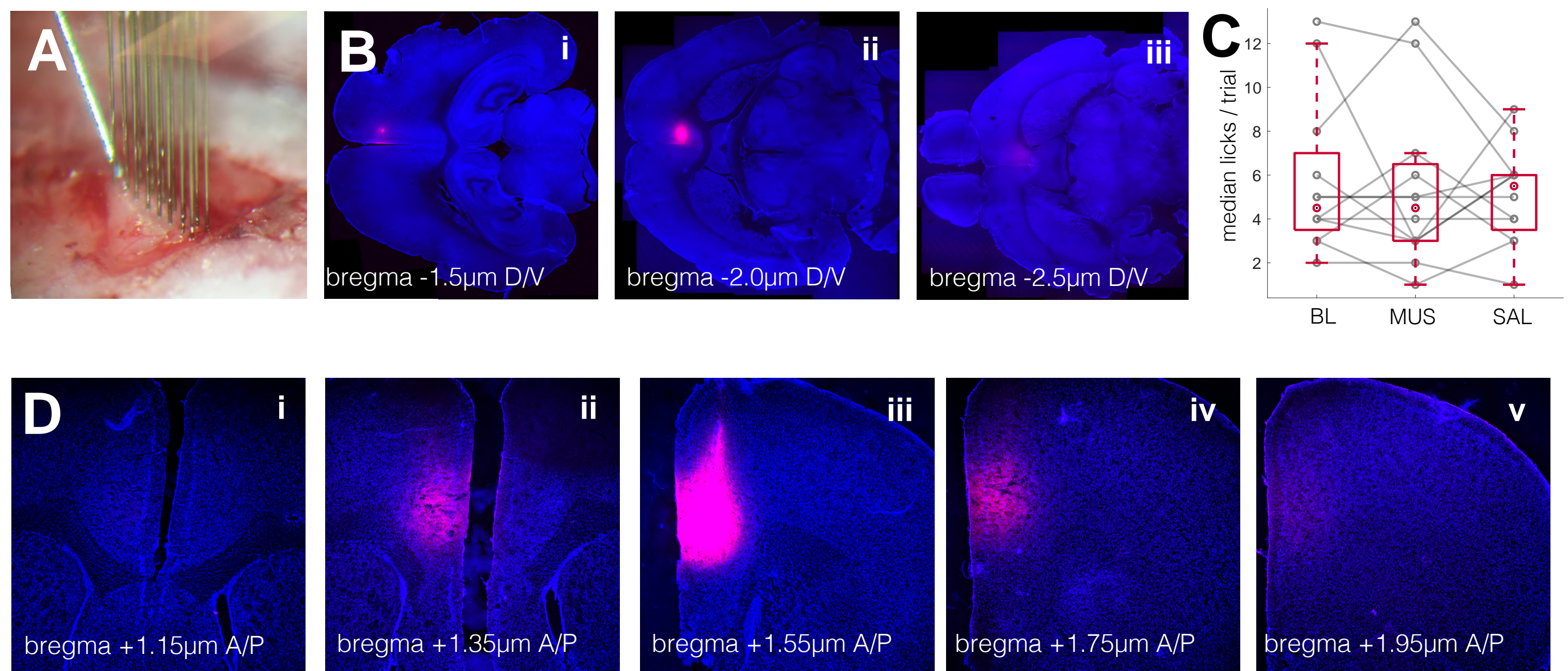


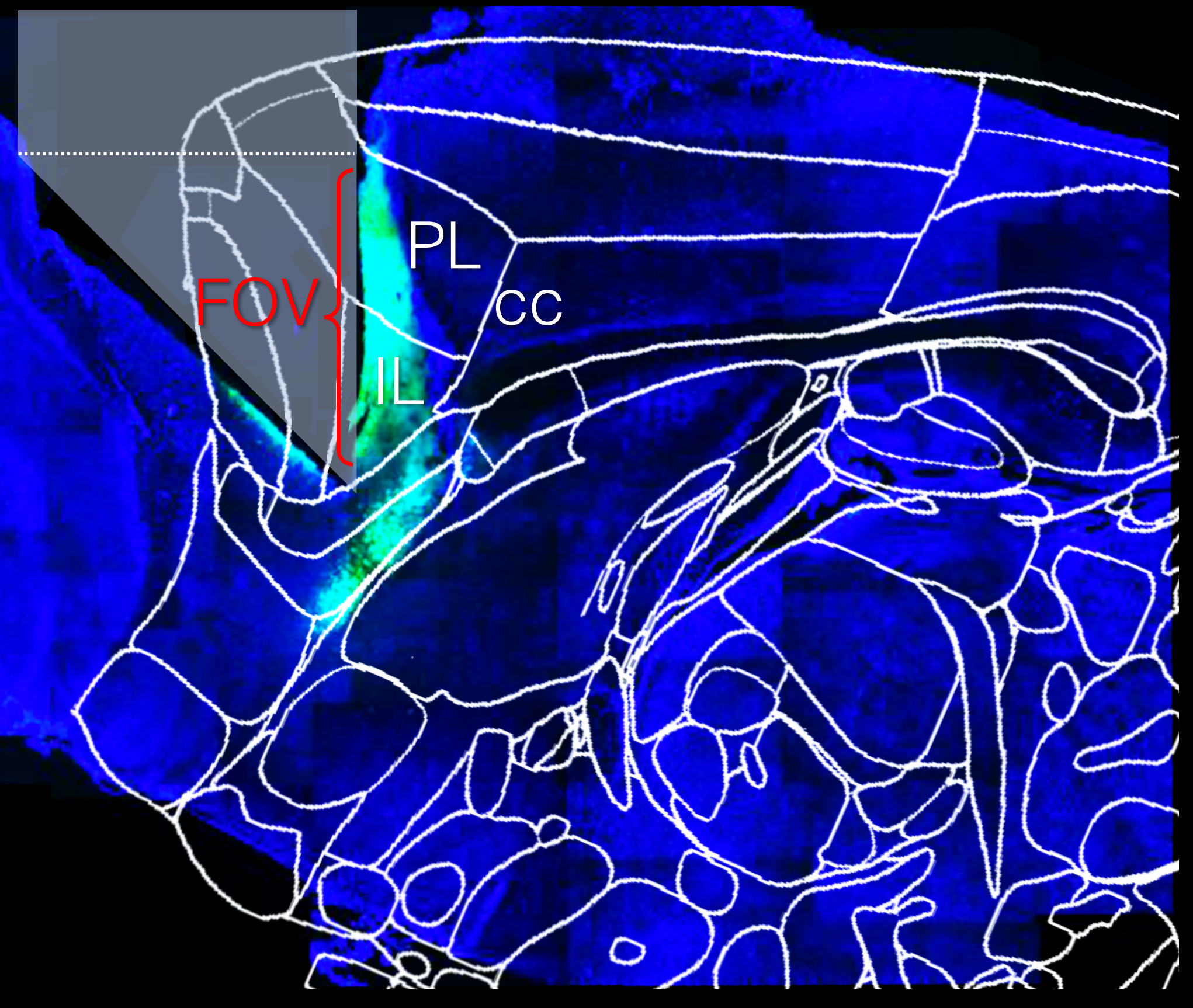




\section{Figure S3}

A animal t284, PFC-MDT
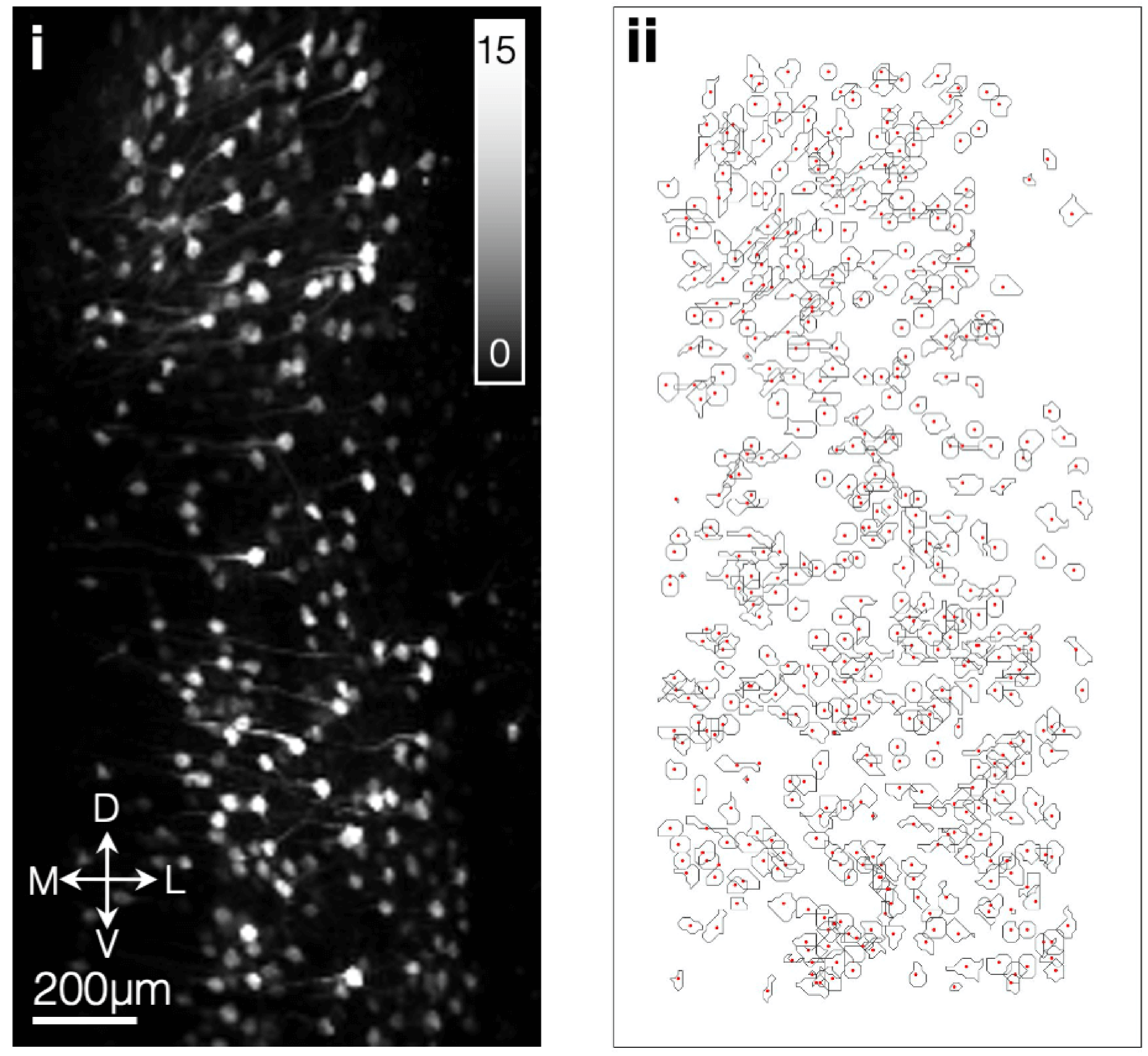

$\bigcirc$ ROI $\bigcirc$ nearest ROI $\bigcirc$ neuropil iii
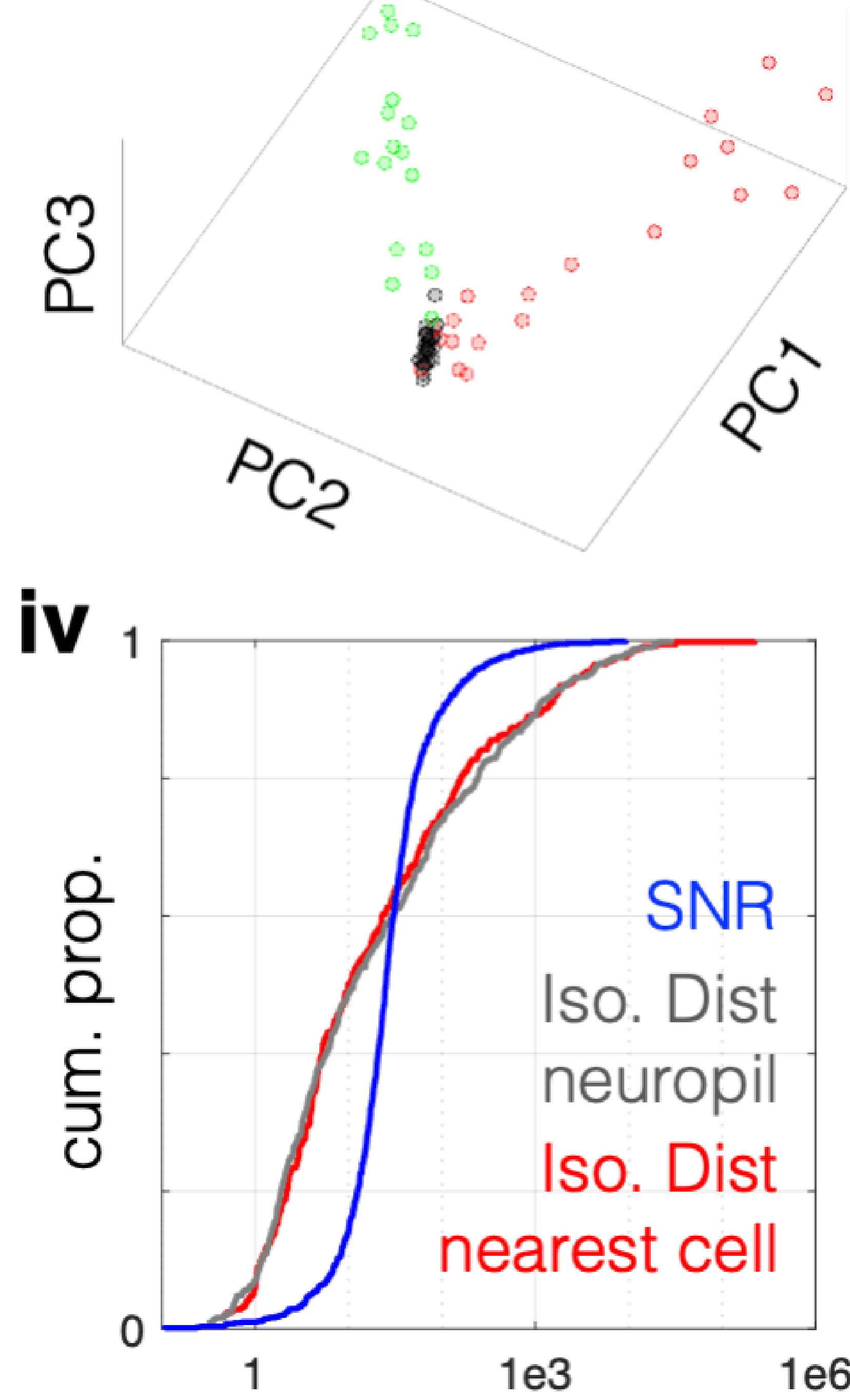

B animal t286, PFC-MDT
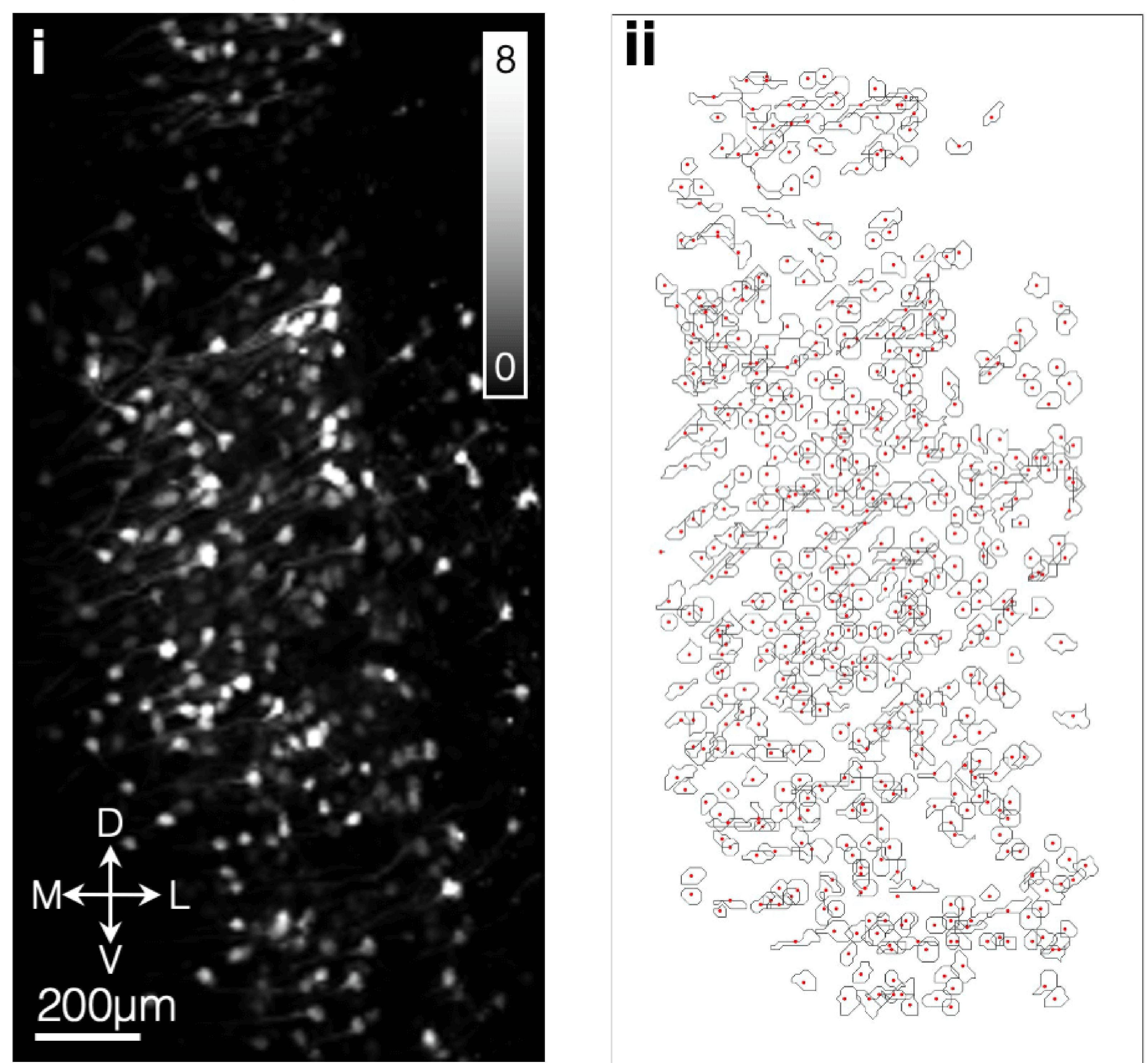

iii

$\bigcup_{0}^{n}$
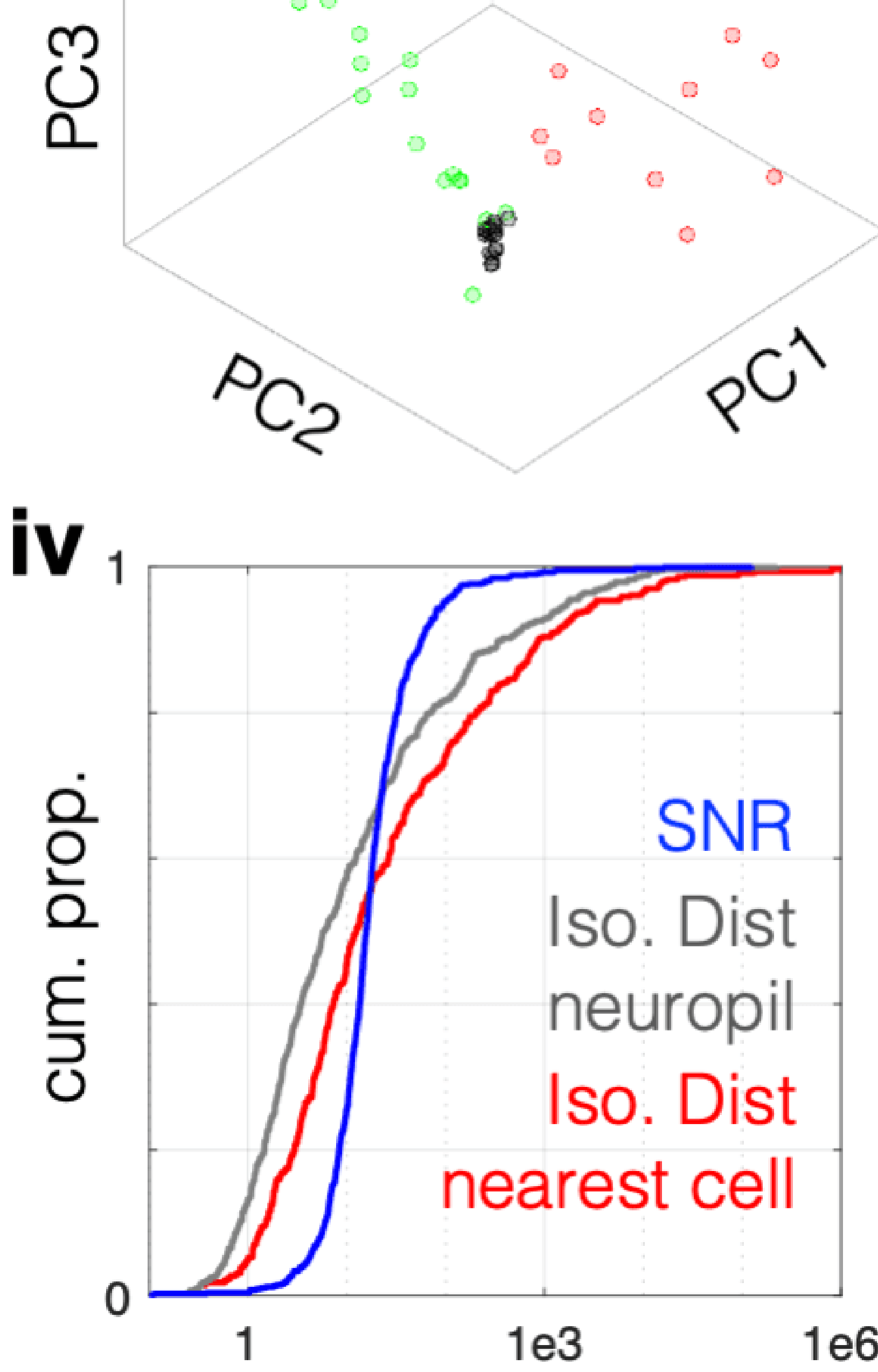


\section{Figure S4}

A animal t382, PFC-VMS
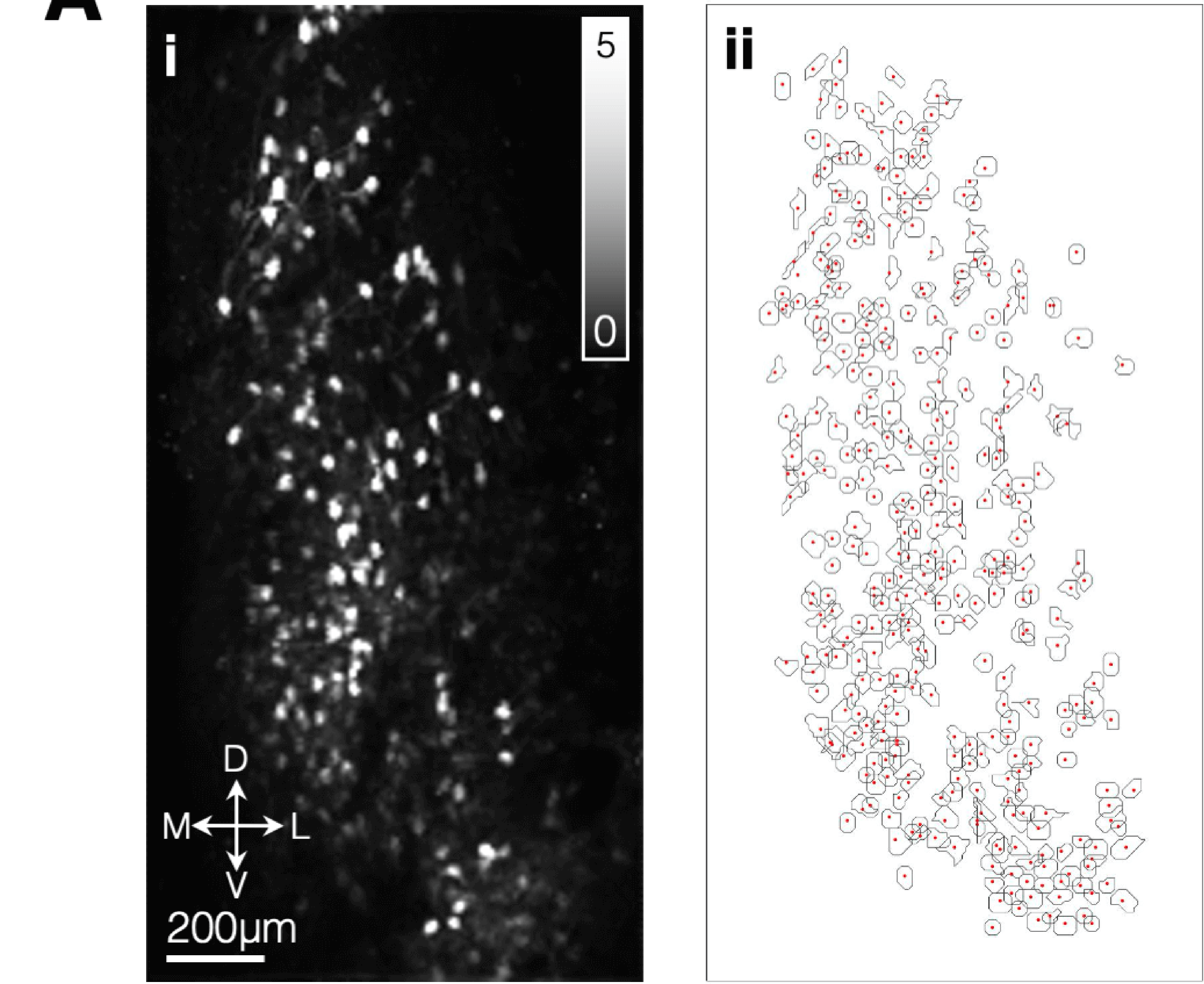

$\bigcirc$ ROI $\bigcirc$ nearest ROI $\bigcirc$ neuropil

iii

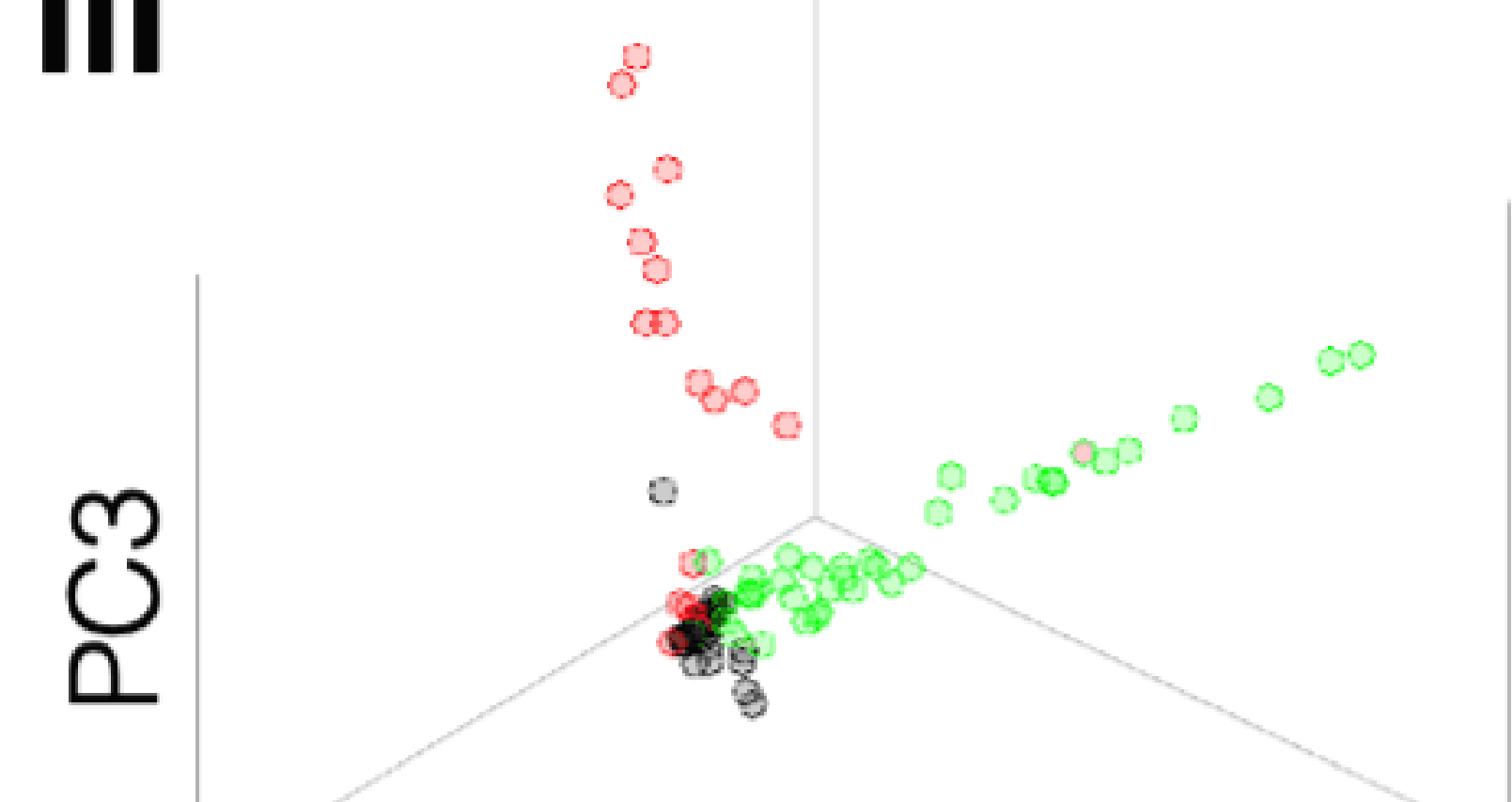

B
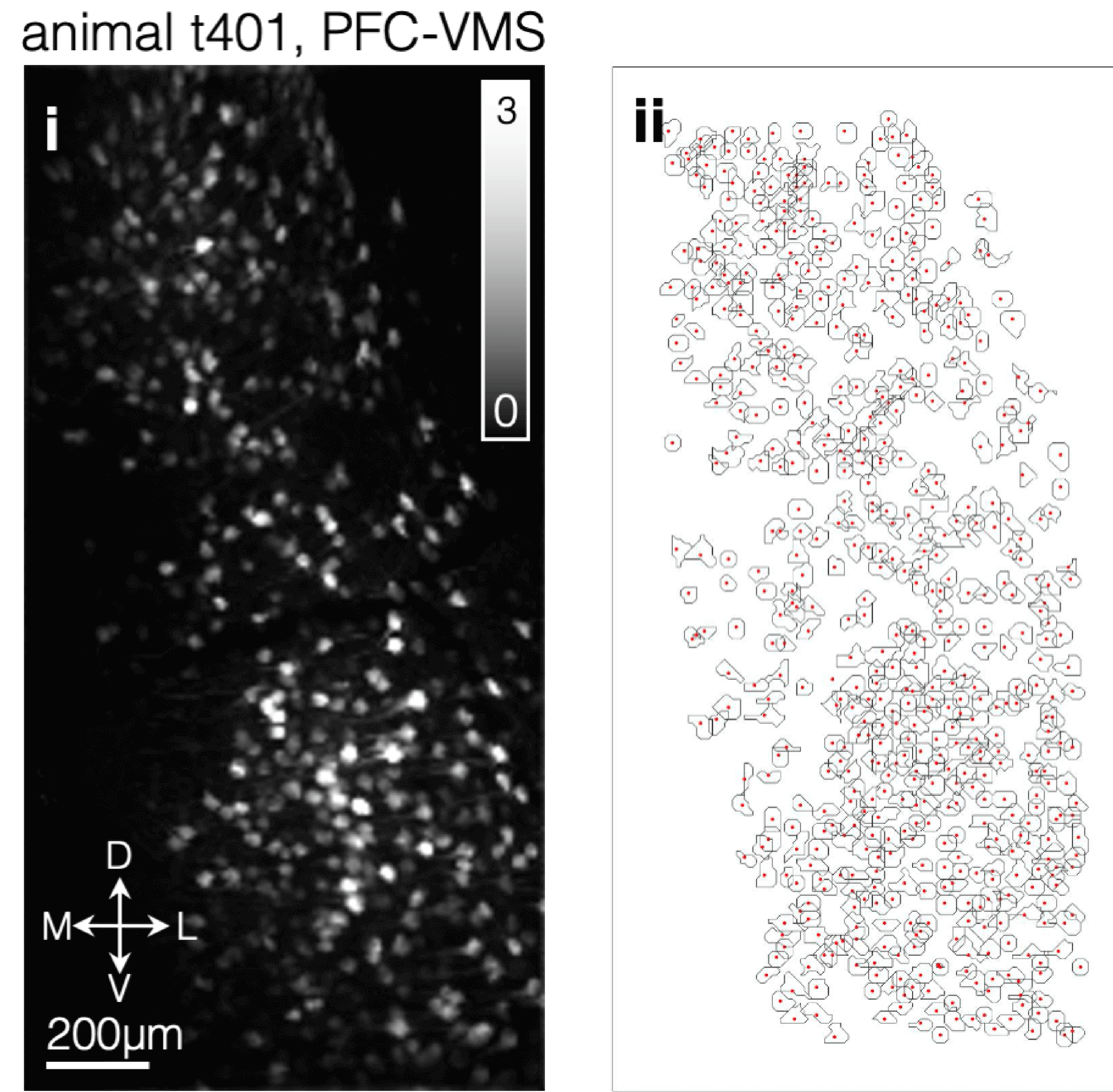

iii
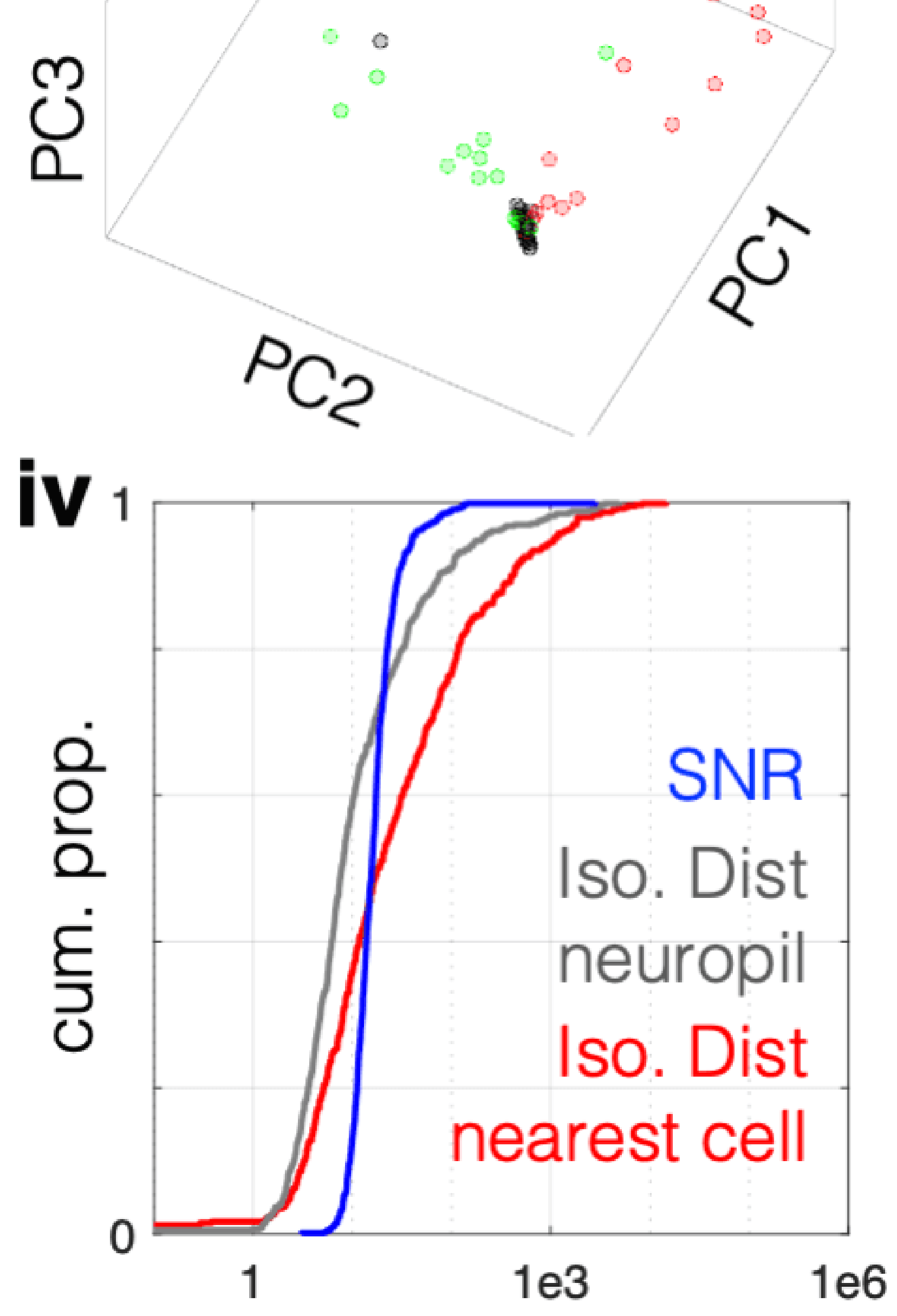


\section{Figure S5}

A animal t284, PFC-MDT

\section{i session 1 session 2}

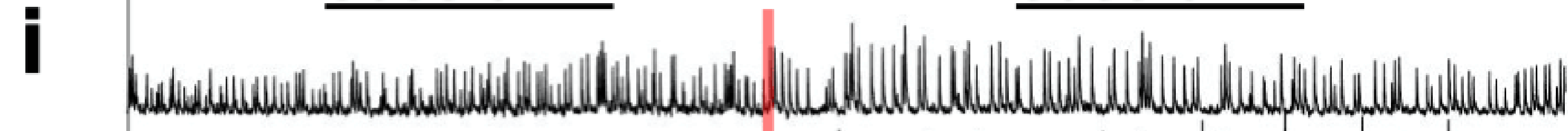

\$

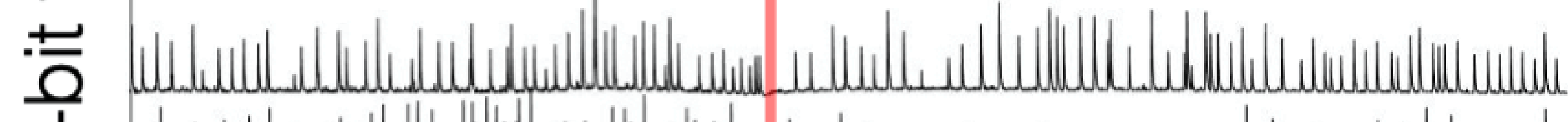

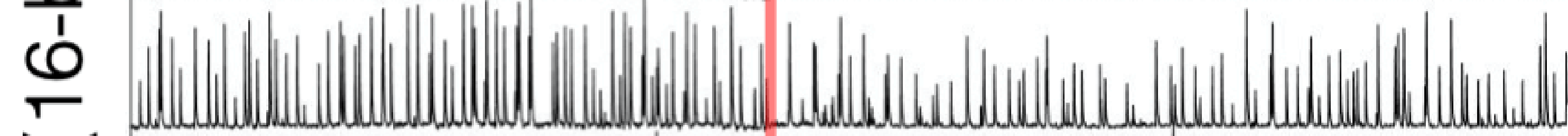

¿

¿

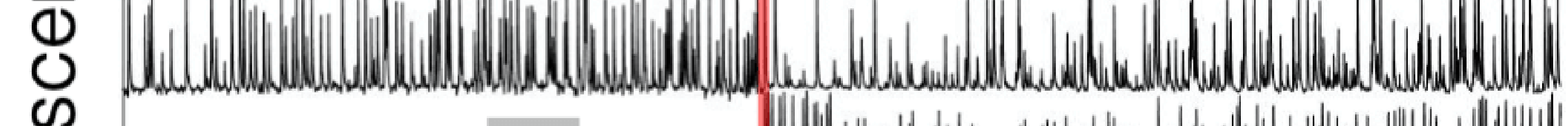

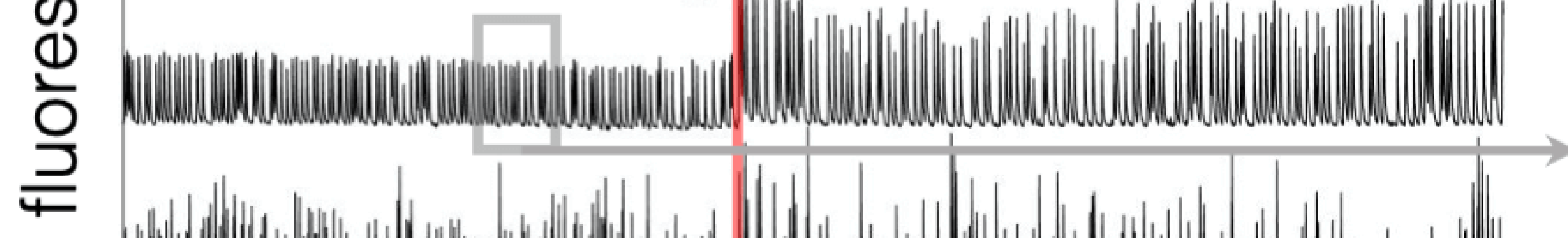

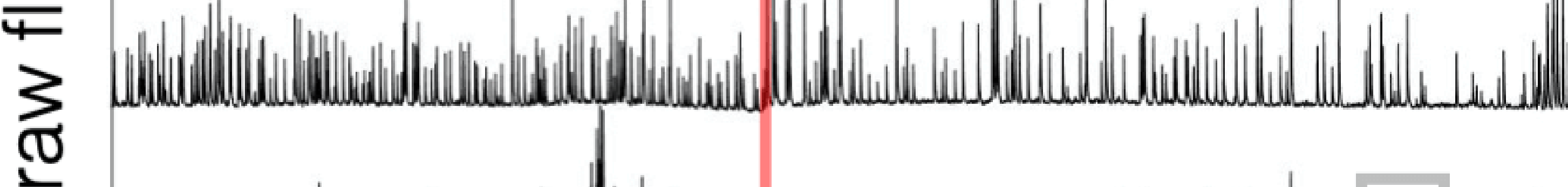

৪)

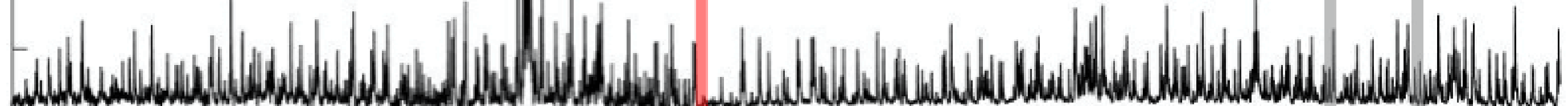

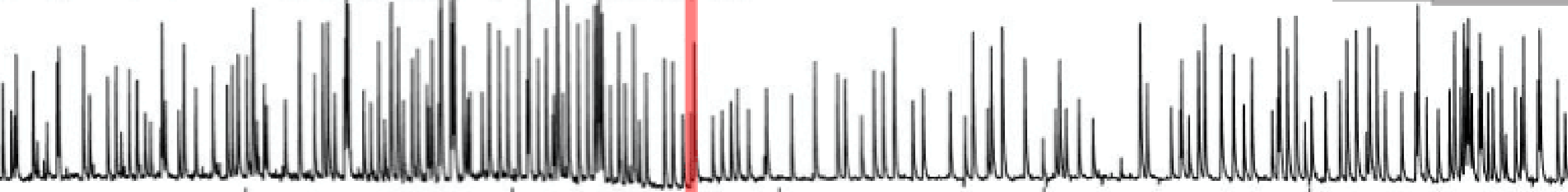
$\begin{array}{llllll}0 & 2 \mathrm{k} & 4 \mathrm{k} & 6 \mathrm{k} & 8 \mathrm{k} & 10 \mathrm{k}\end{array}$

B animal t286, PFC-MDT

i

i session $1 \quad \frac{\text { session } 2}{\text { session } 3}$

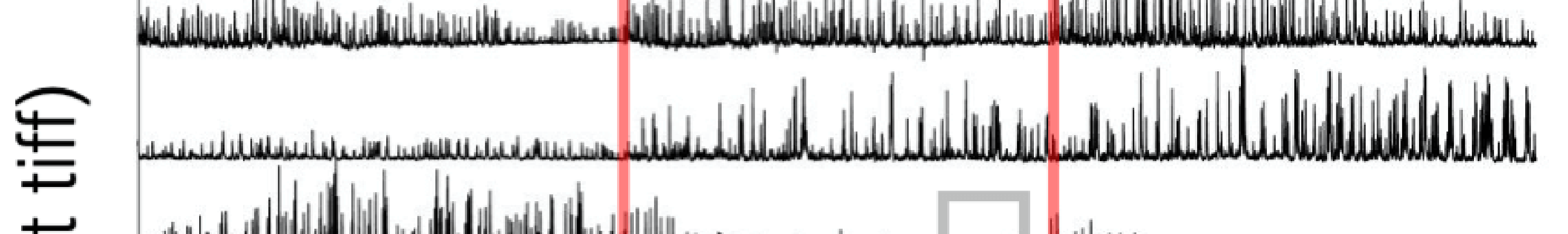

:

ف․

¿

D

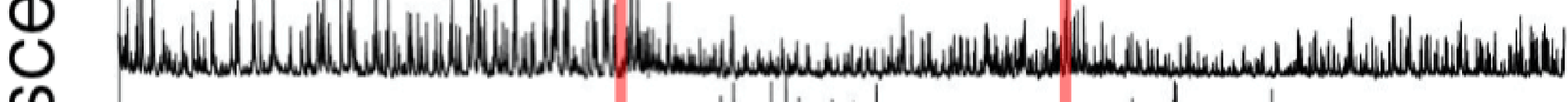

o

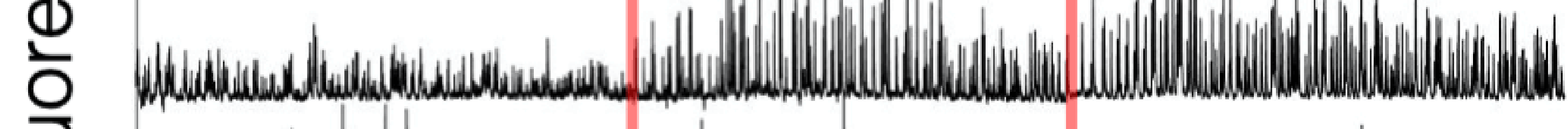

$\stackrel{2}{2}$

苂

:

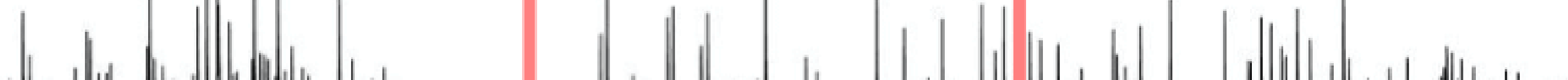

Fin

.

I

0

0

$4 \mathrm{k}$

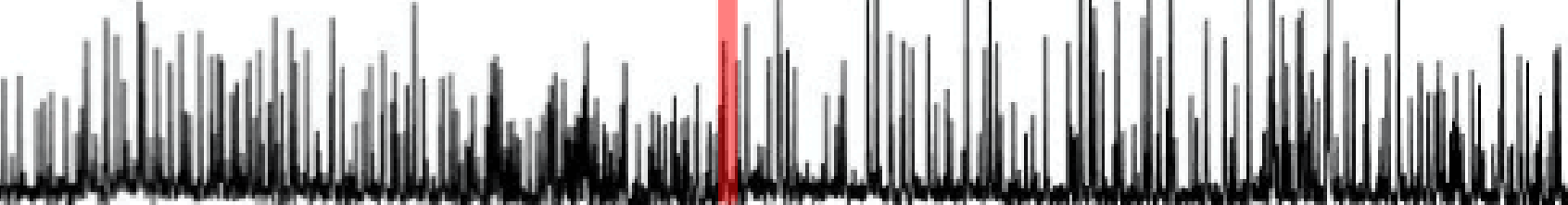

$8 \mathrm{k} \quad 12 \mathrm{k} \quad 16 \mathrm{k} \quad 20 \mathrm{k}$ time (sec) ii $\square$ raw signal $\square \Delta f / f \square$ spike prob.

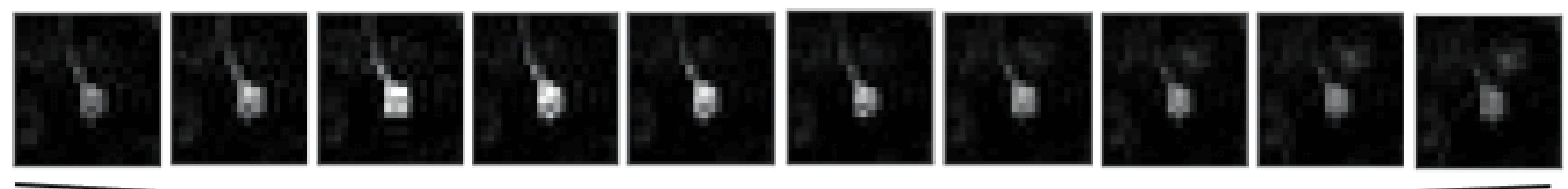

임

옝

인

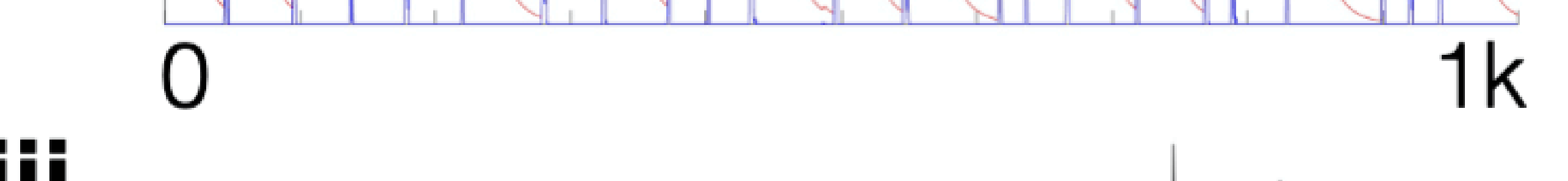

iii

잉

잉

잉

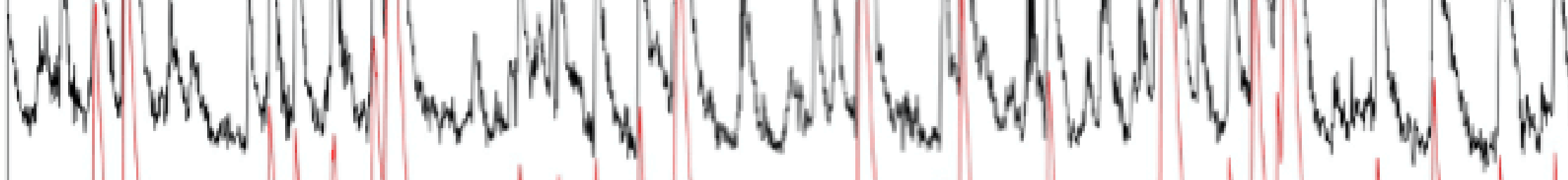

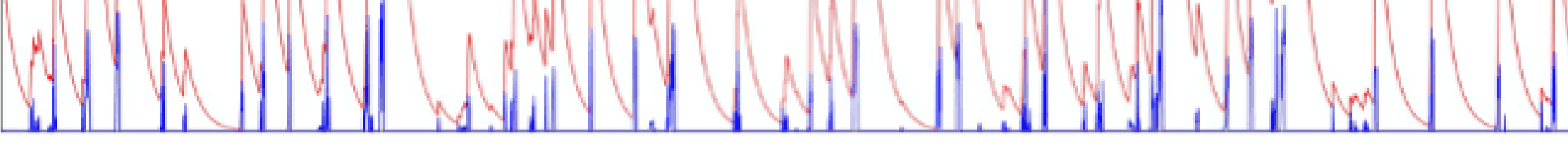

0

$1 \mathrm{k}$

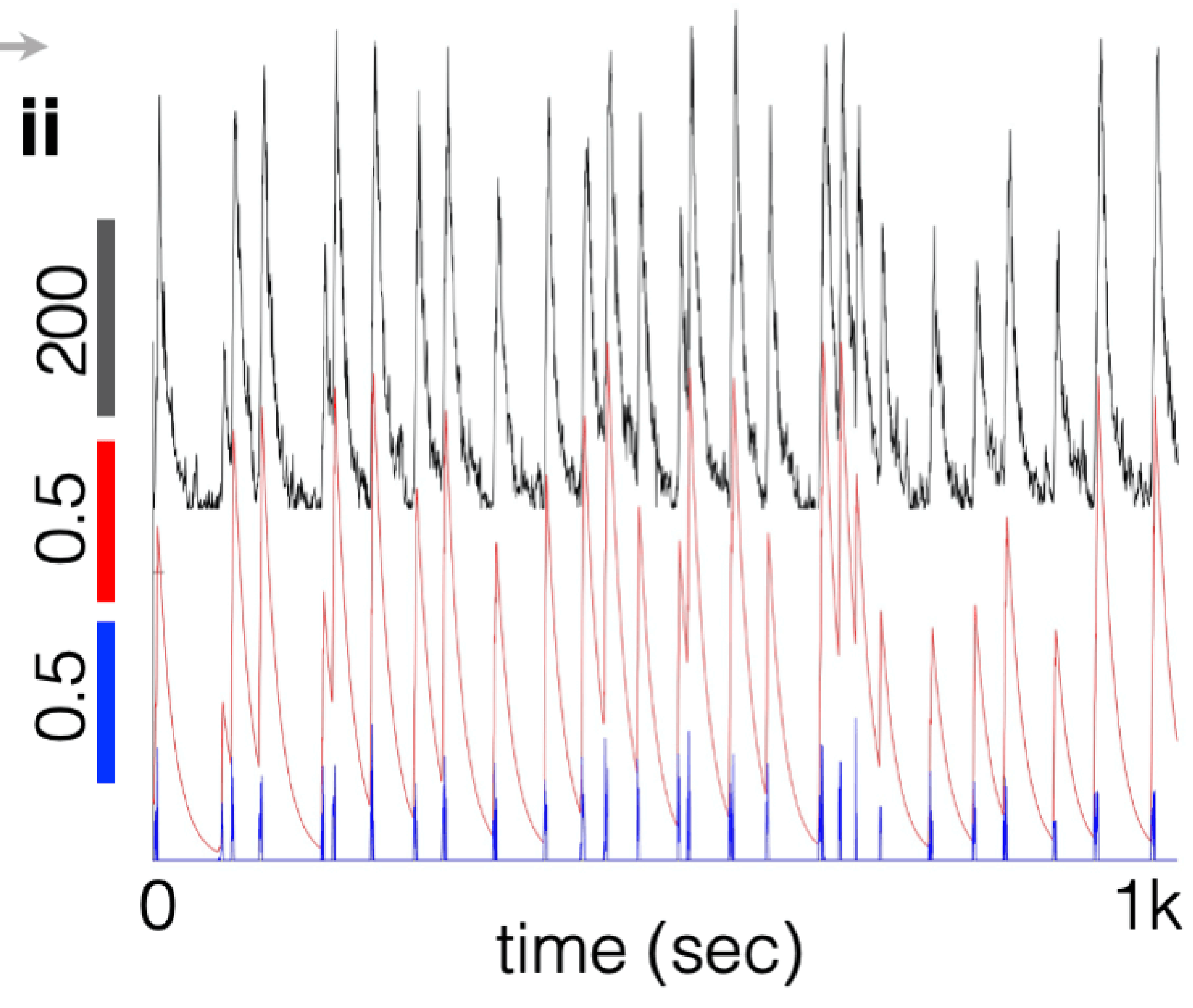




\section{Figure S6}

A animal t382, PFC-VMS

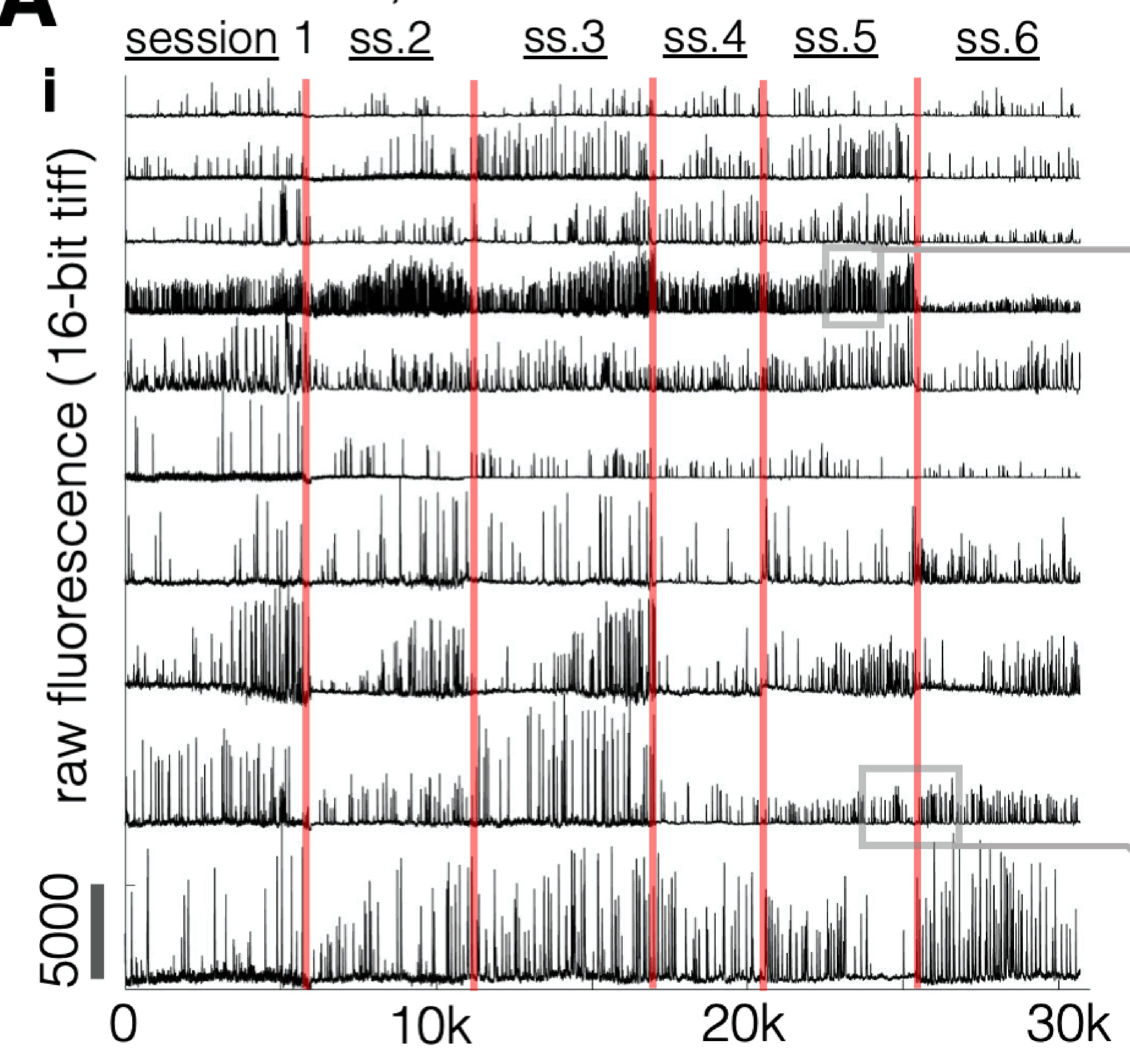

B animal t401, PFC-VMS

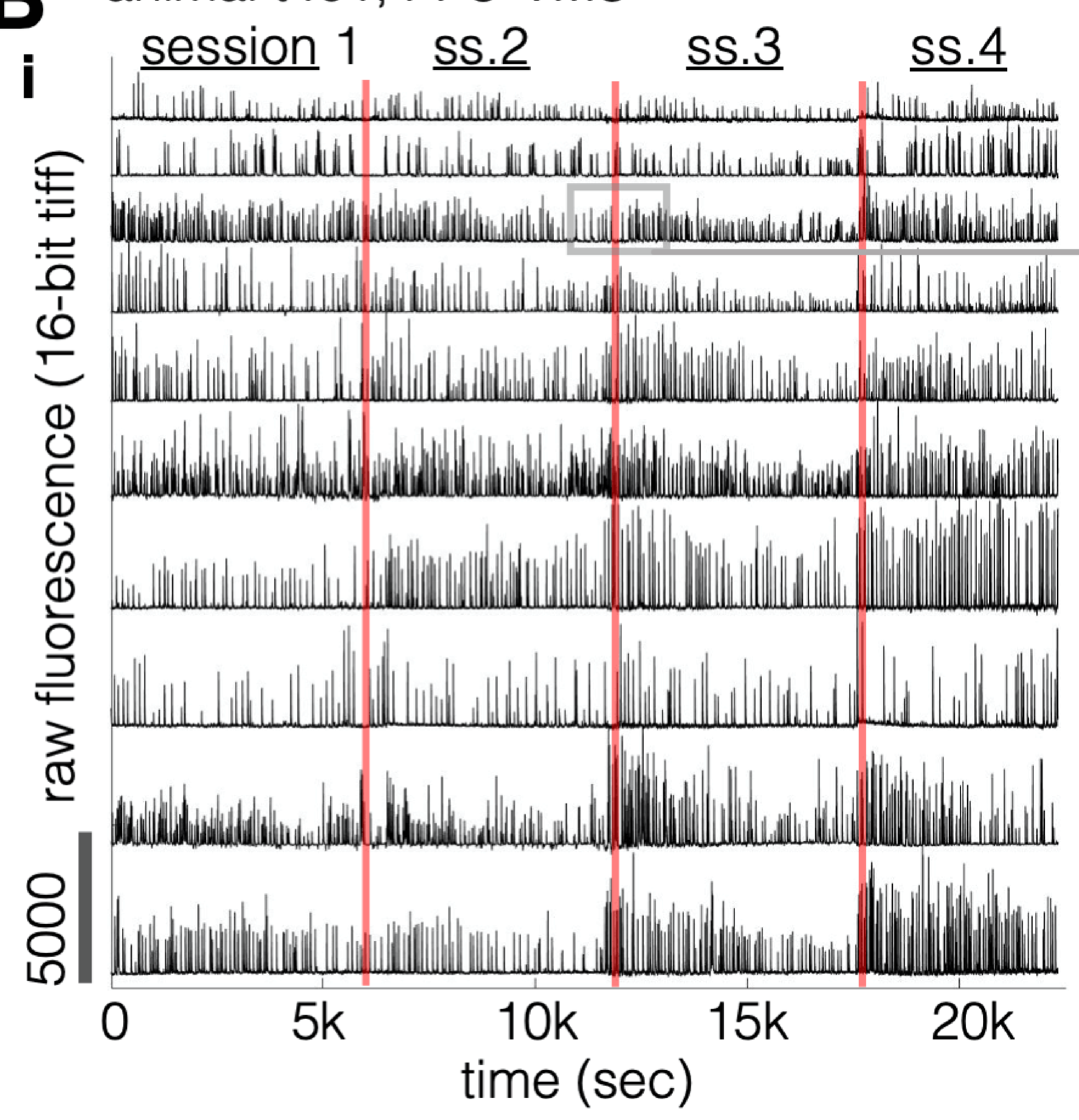

$\square$ raw signal $\square \Delta f / f \square$ spike prob.

ii
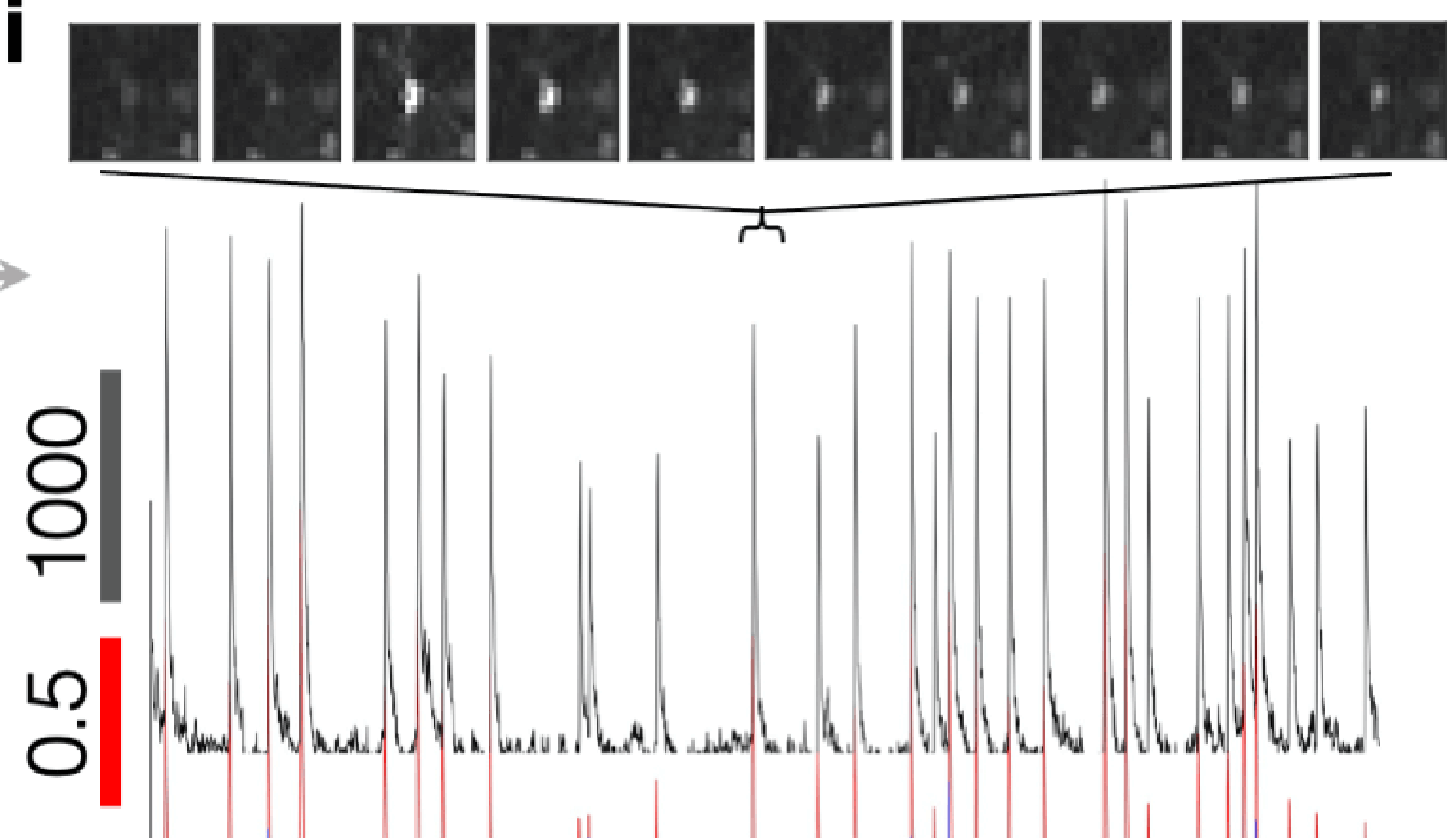

ก)

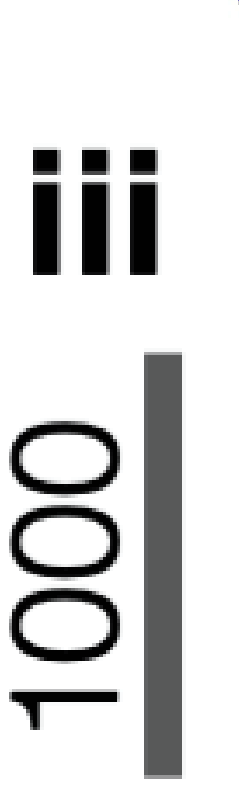

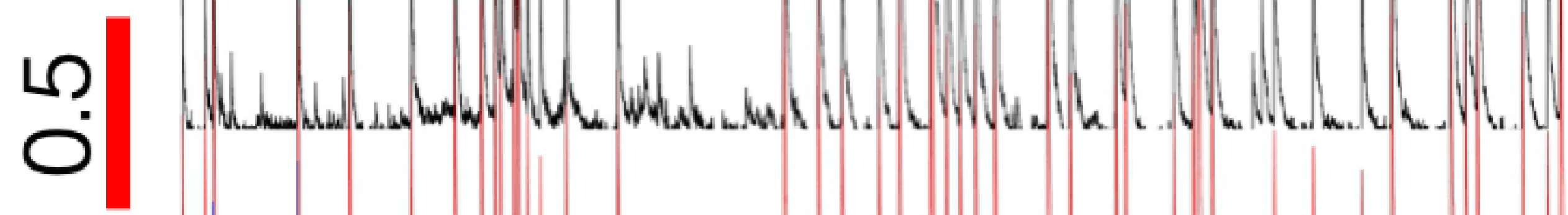

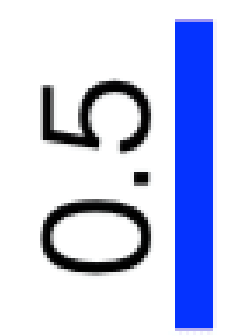

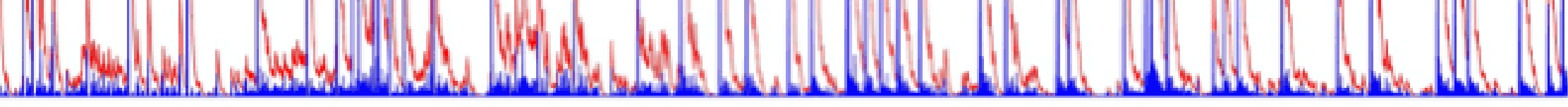

0

$3 \mathrm{k}$

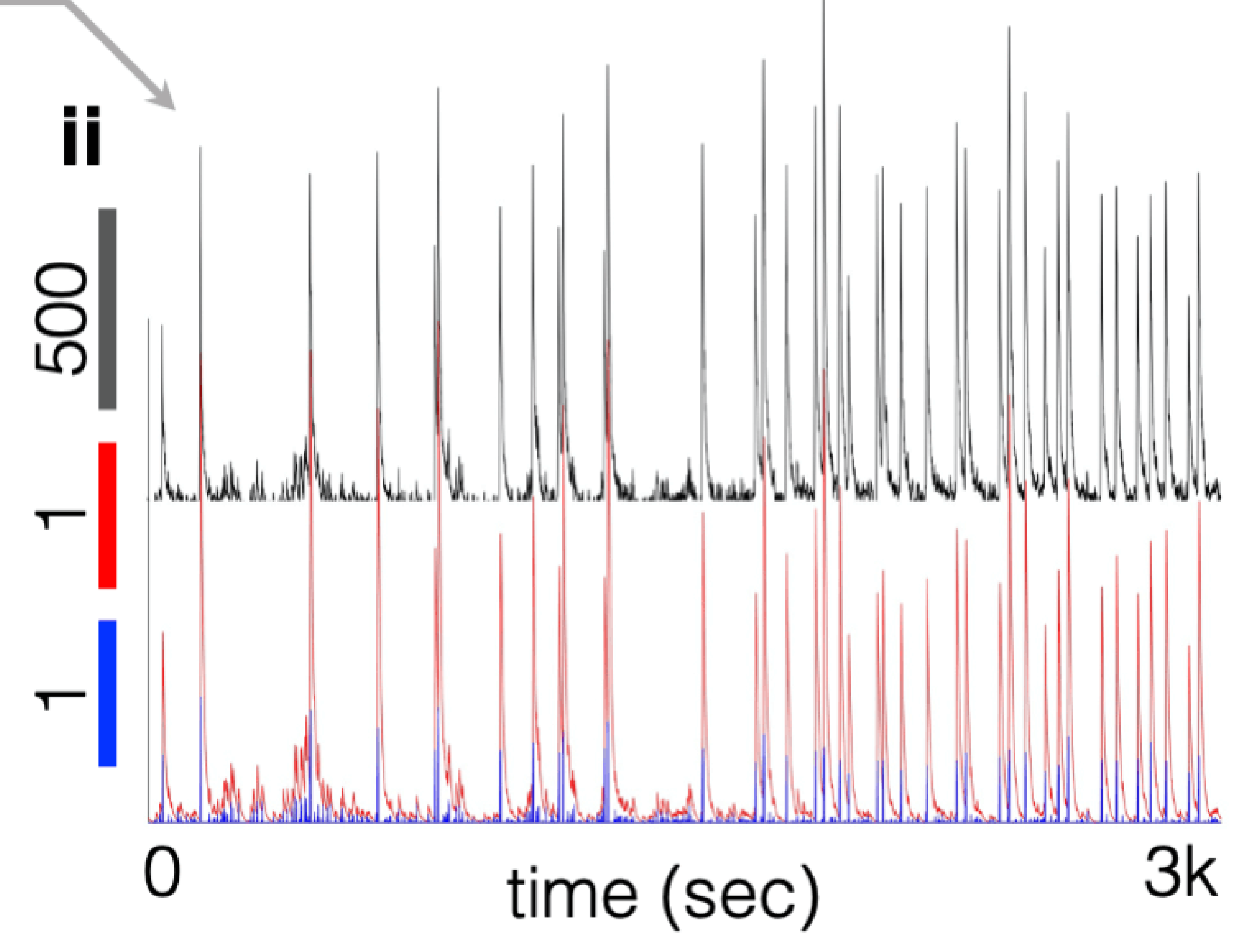




\section{Figure S7}

animal t284, PFC-MDT
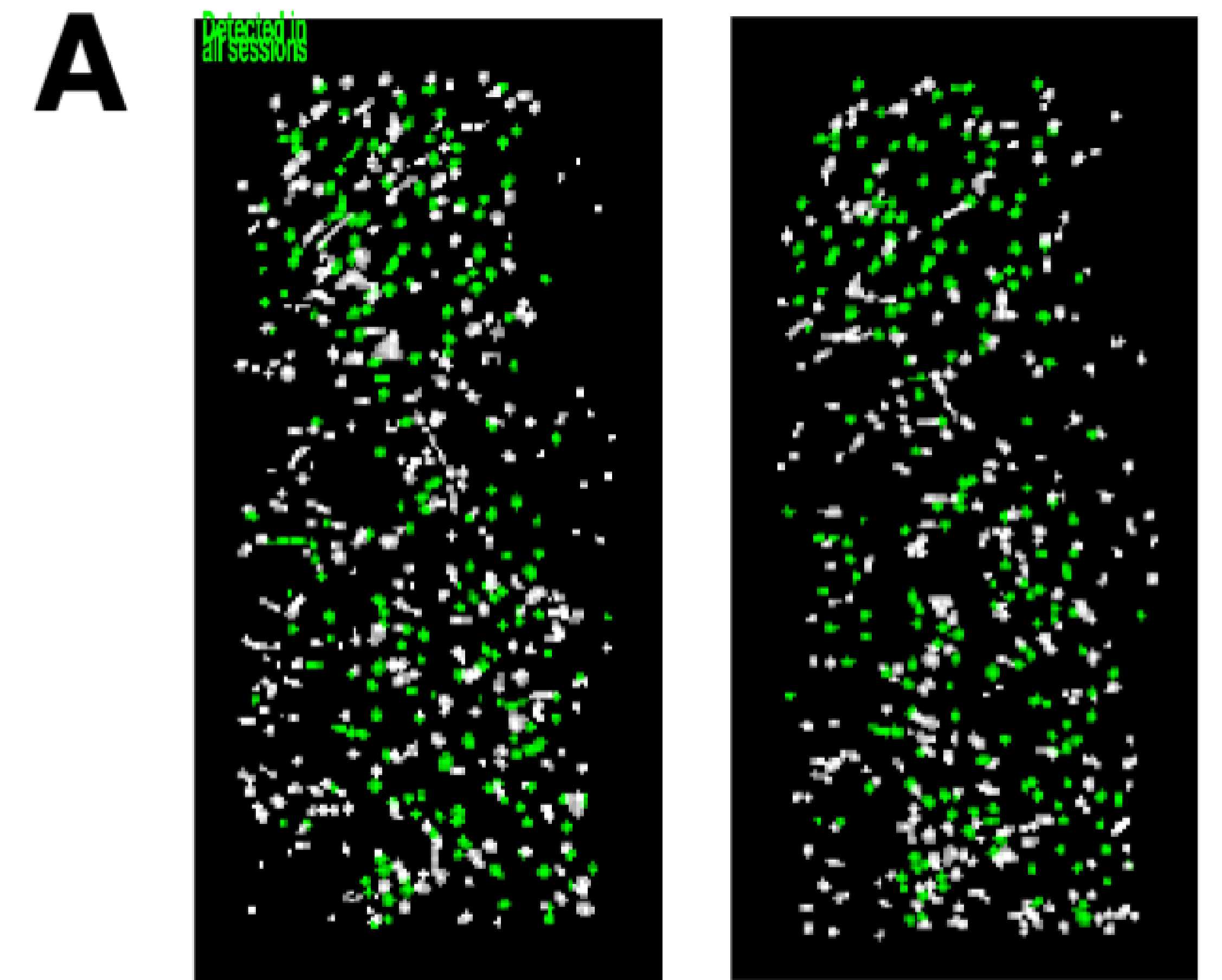

B
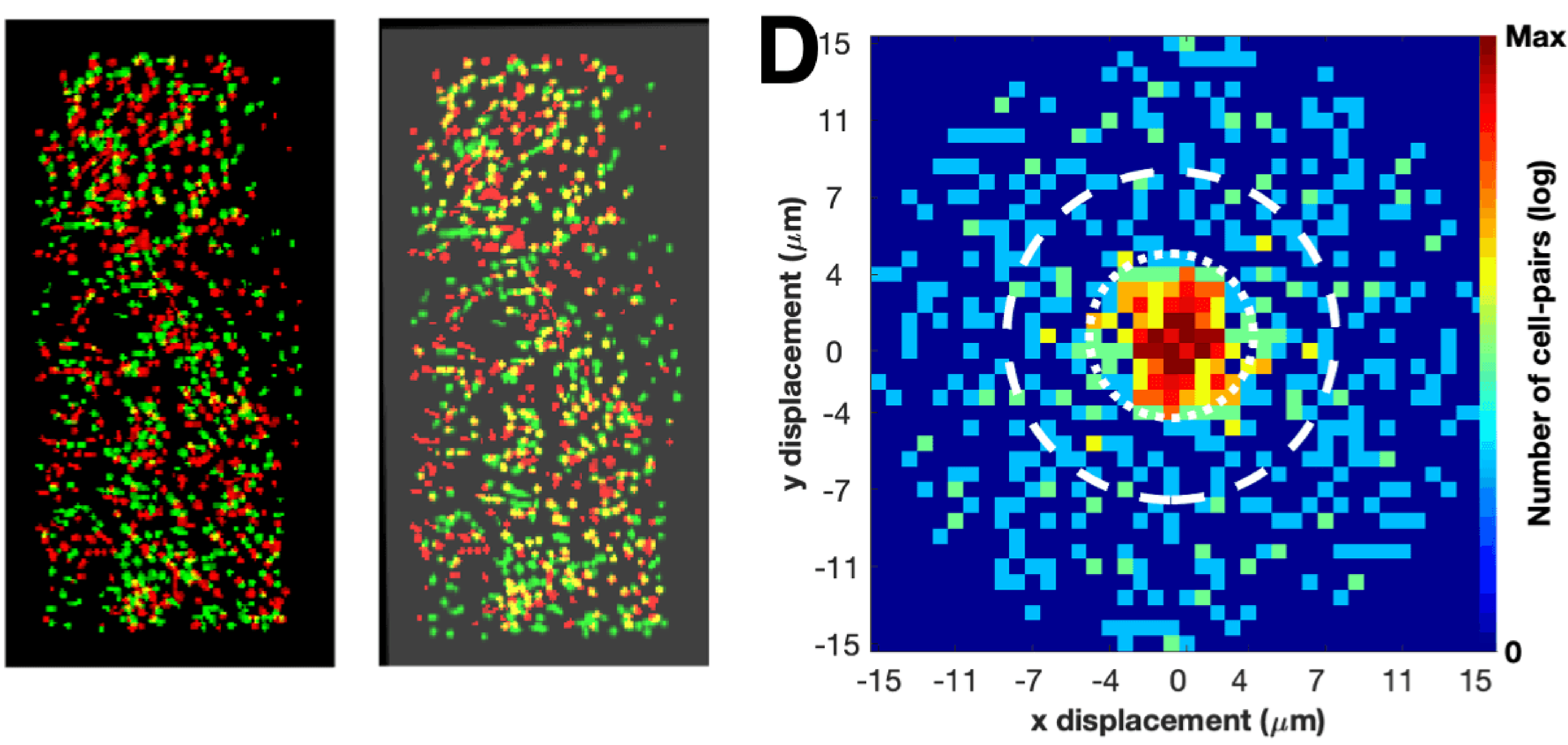

C
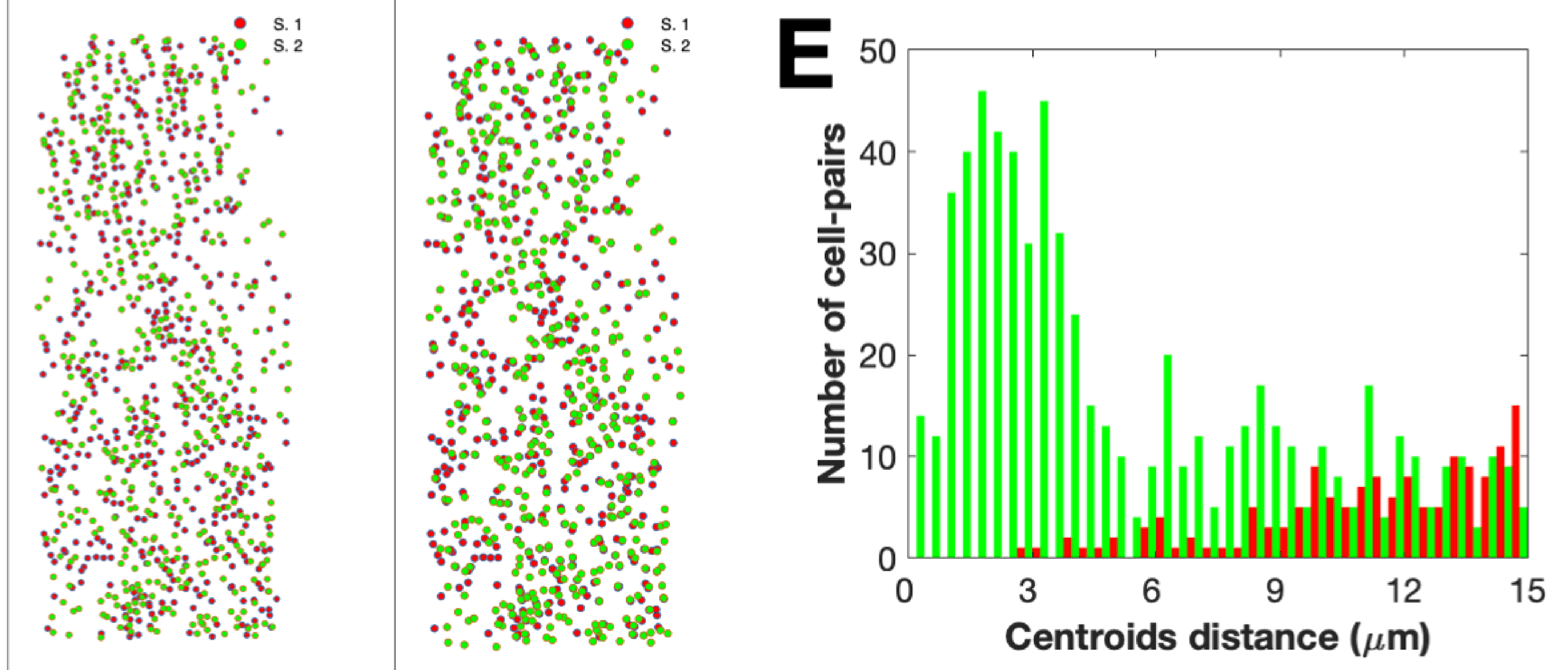


\section{Figure S8}

animal t286, PFC-MDT
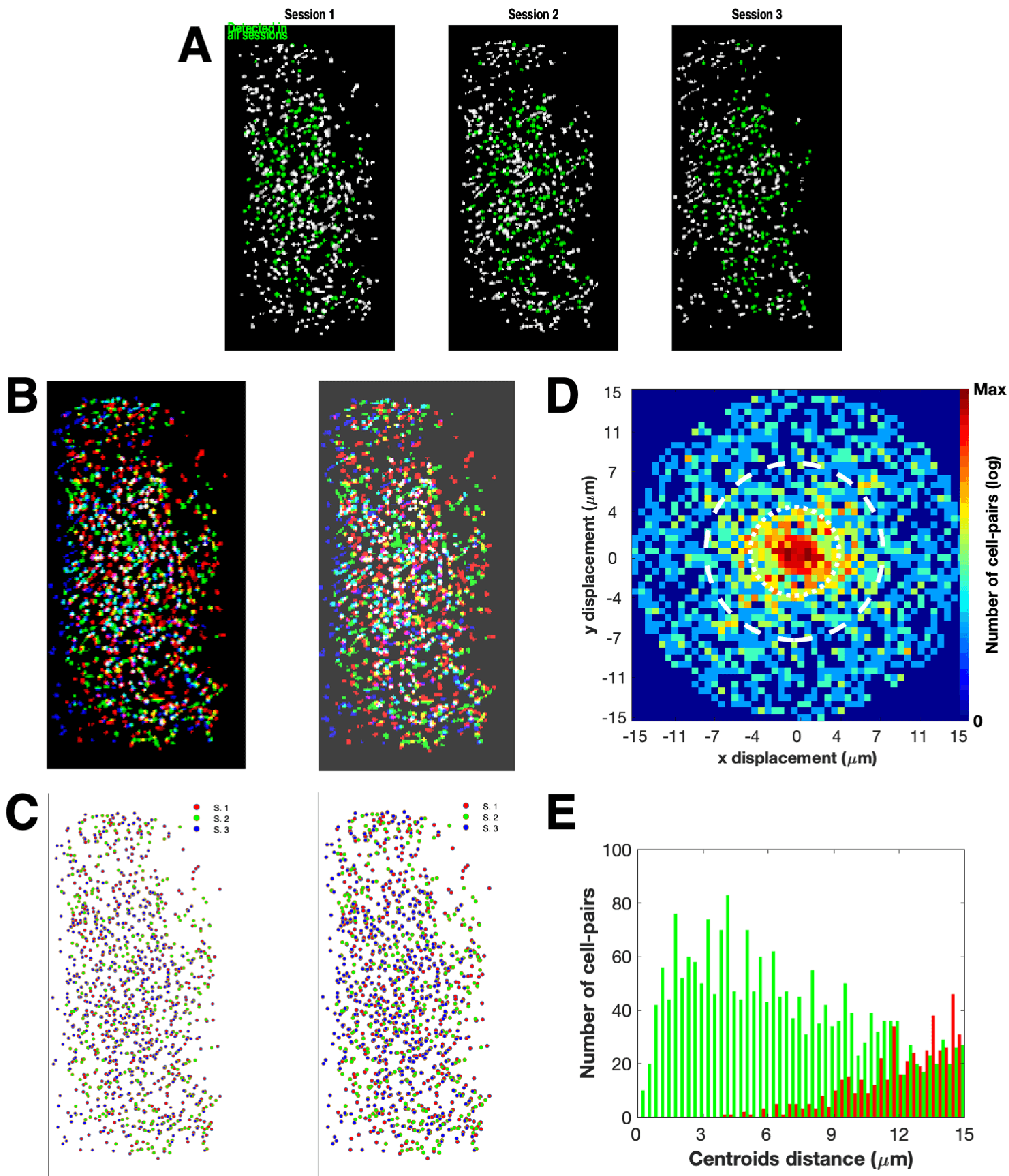


\section{Figure S9}

animal t382, PFC-MDT
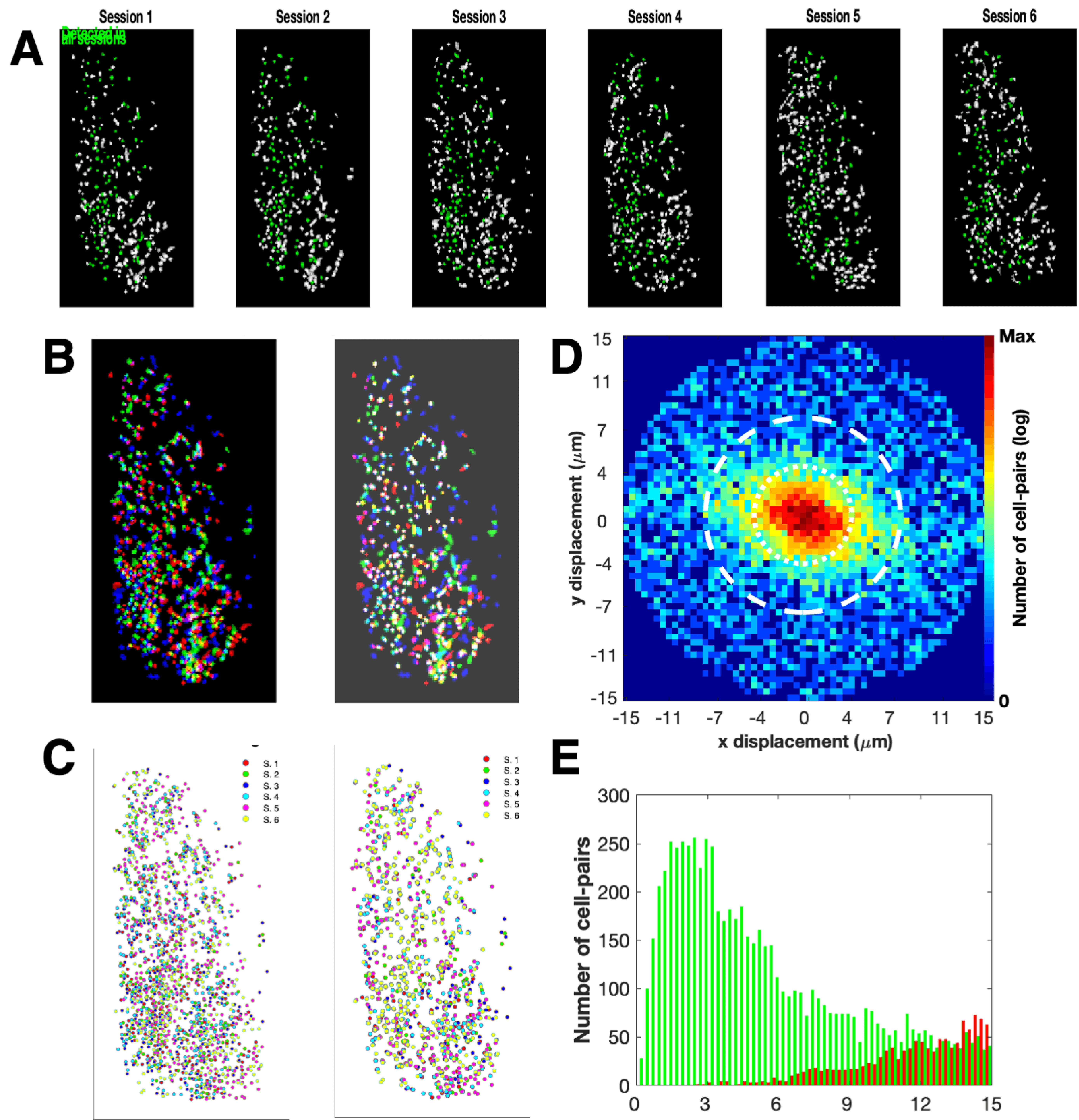

E

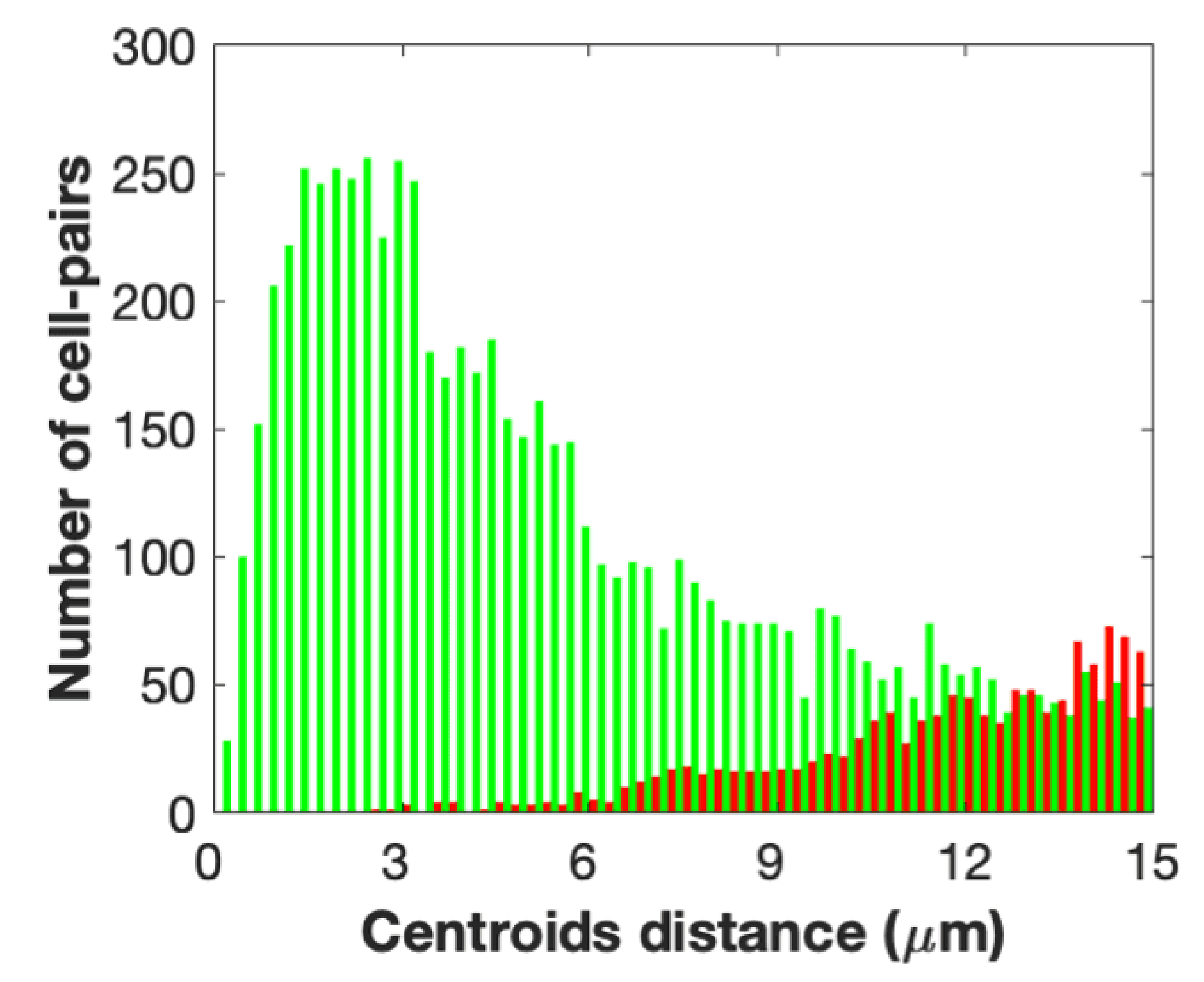




\section{Figure S10}

animal t401, PFC-MDT

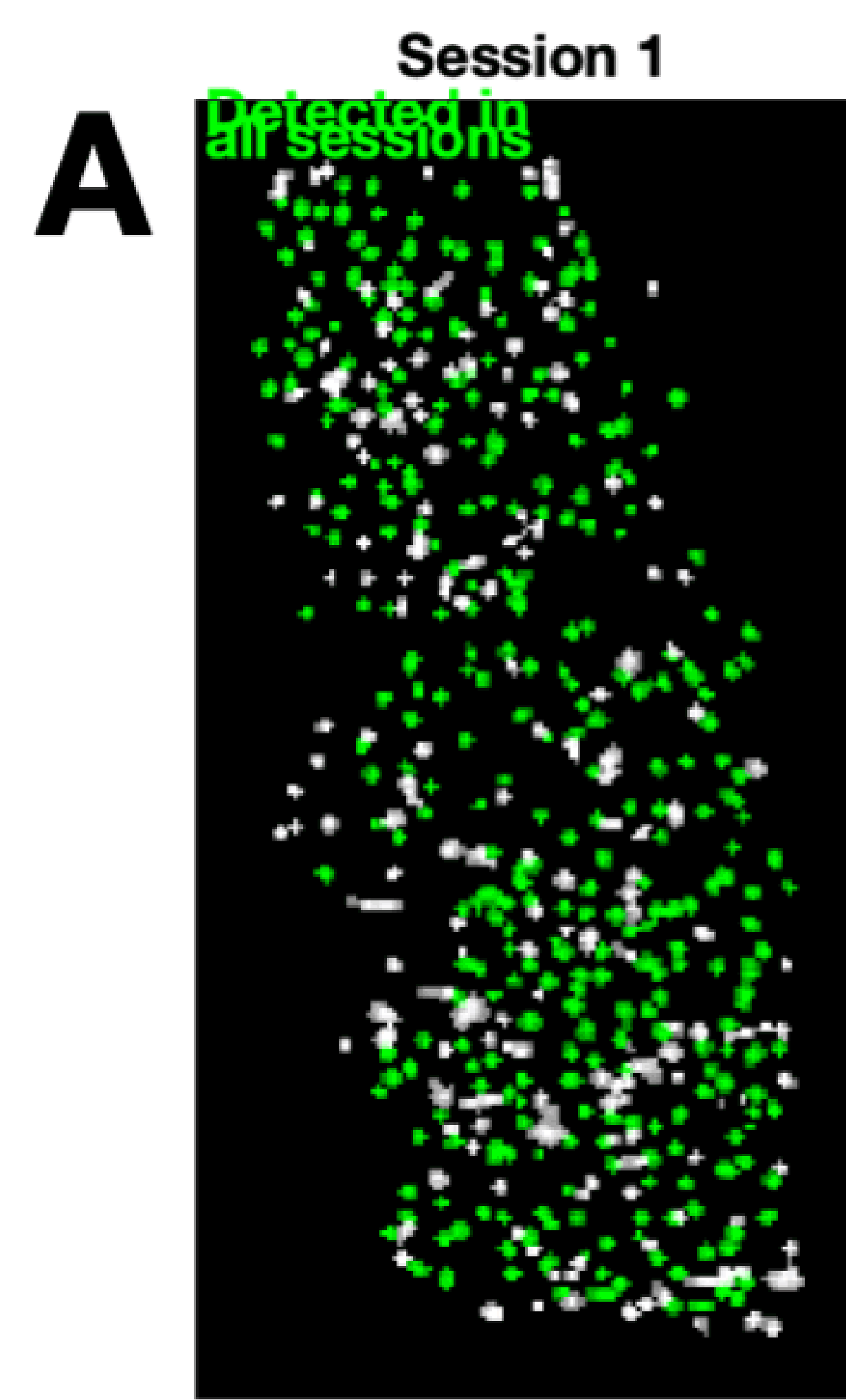

B

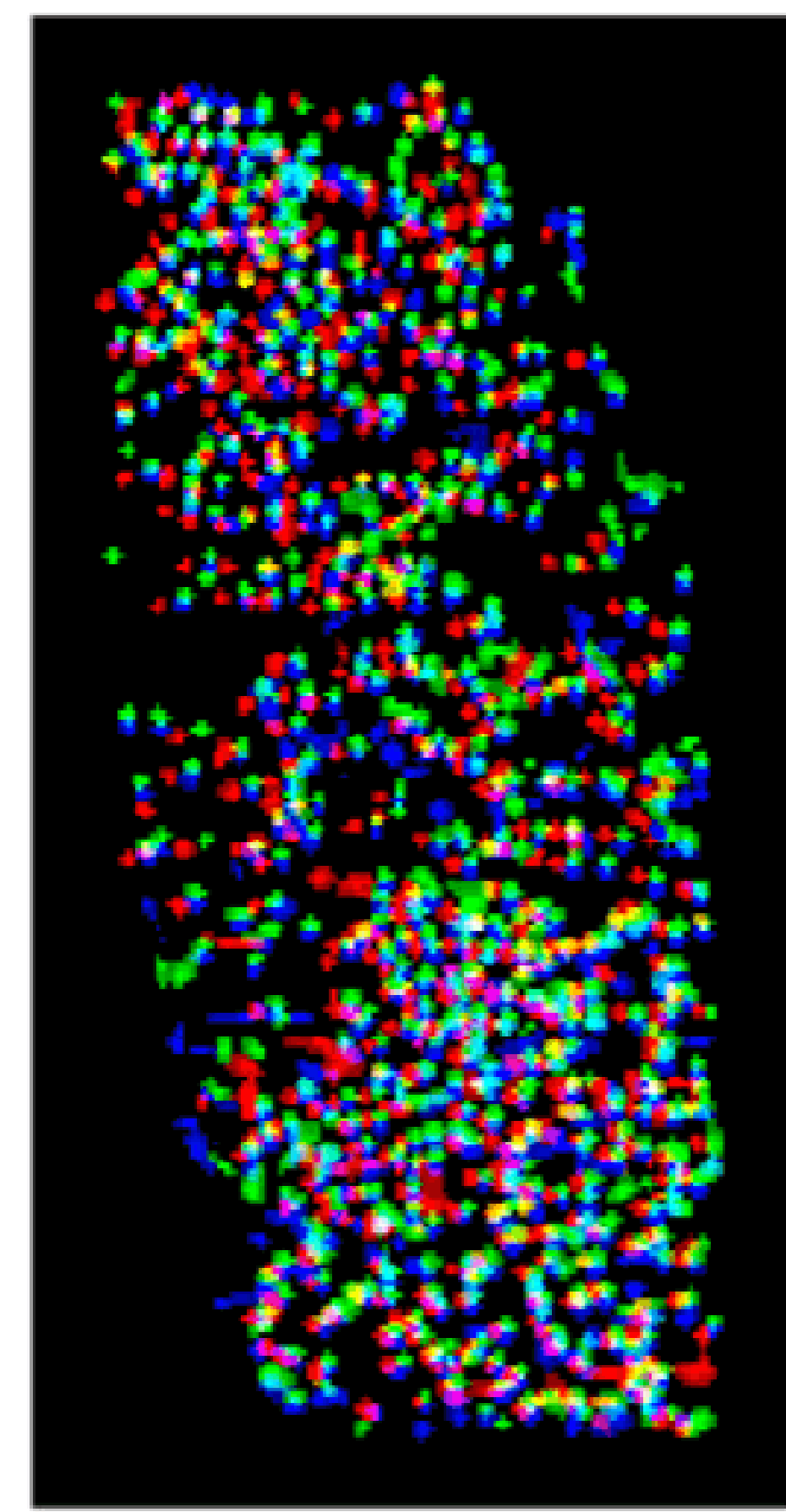

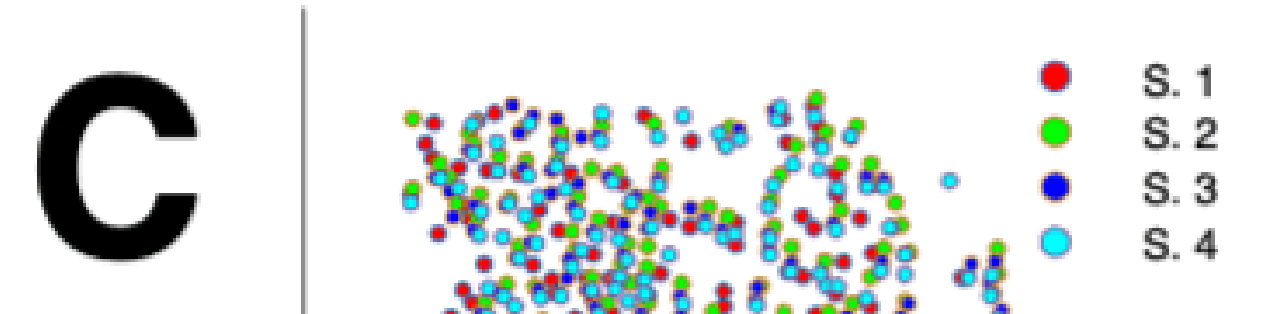

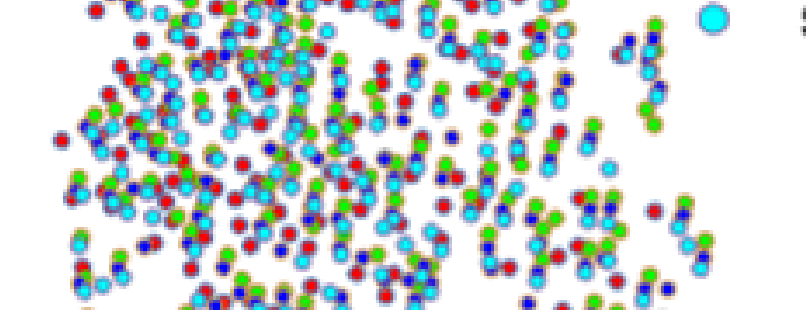

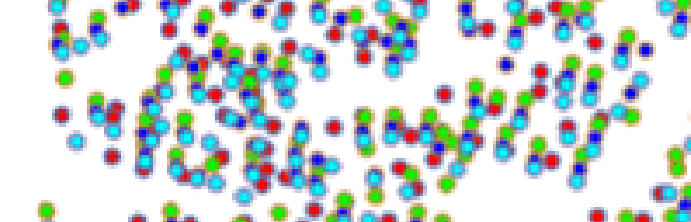

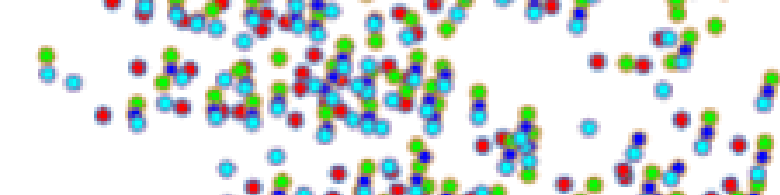

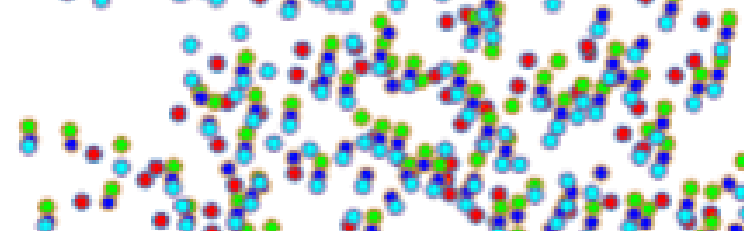

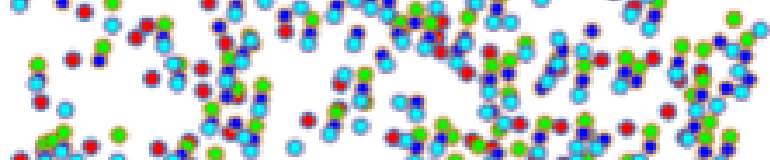
$\because 88^{\circ}$. $\because 4$ \% $\because$ H. $\therefore$ \% รัตำ
Session 2
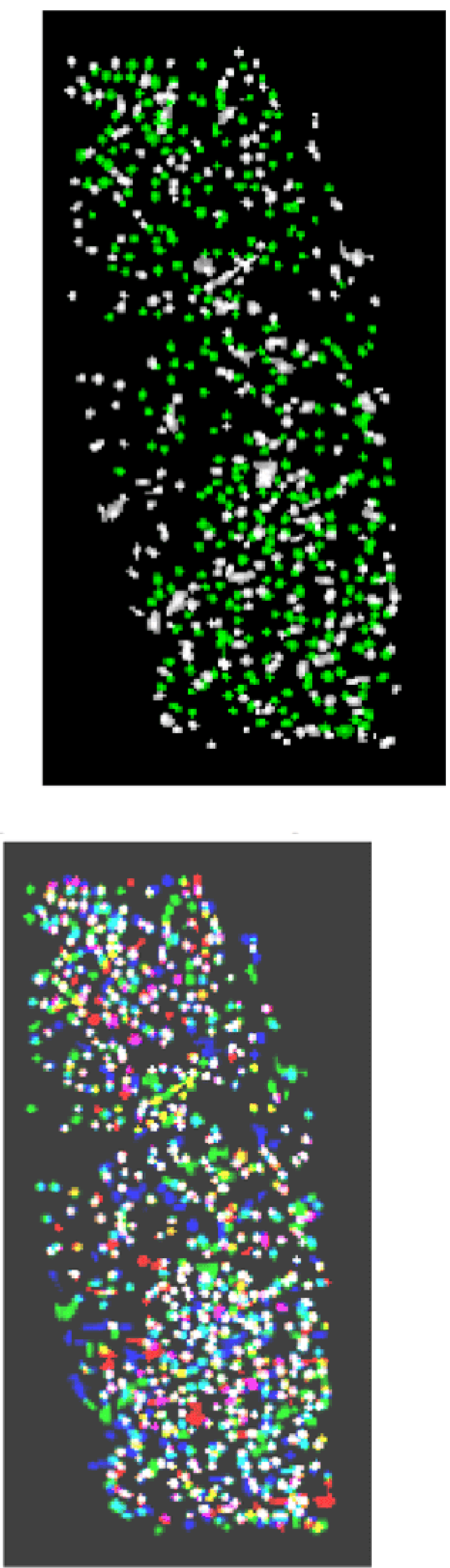

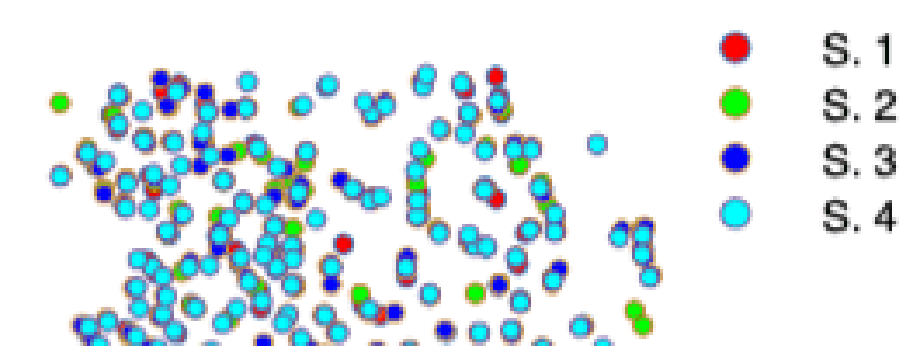

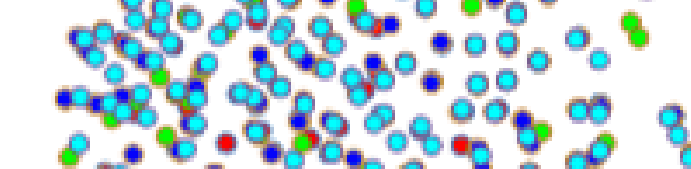

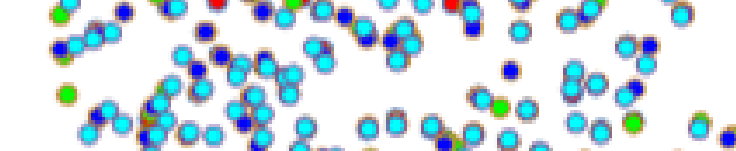

$\therefore 0$

.

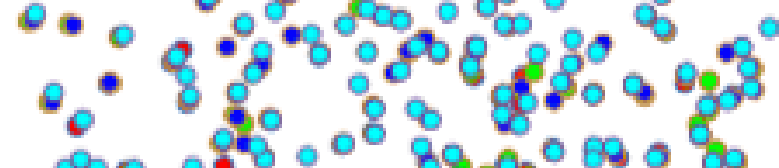

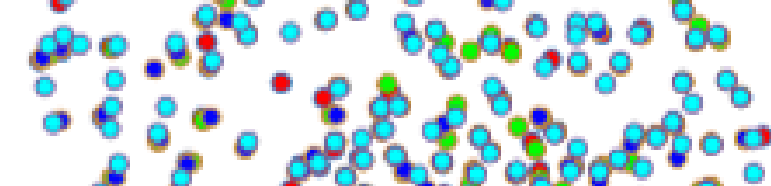

$\because 0 \%$

$\because 0 \%$

\%

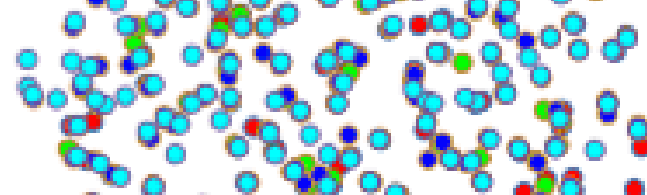

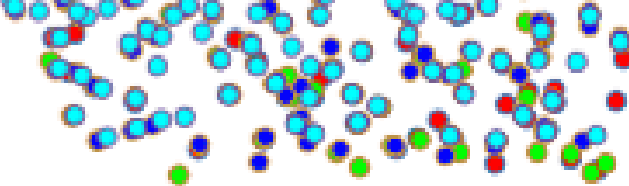

Session 3

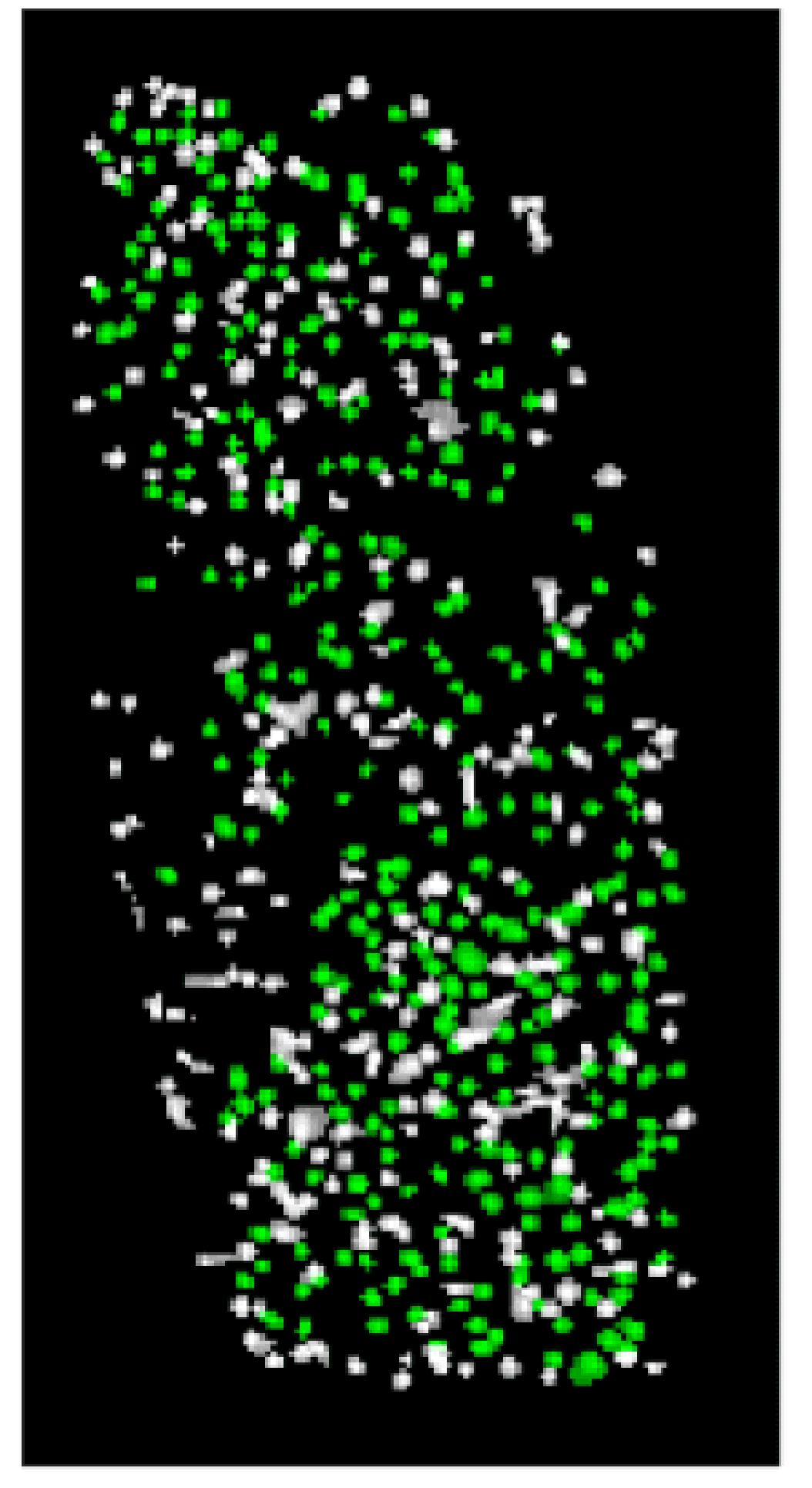

Session 4

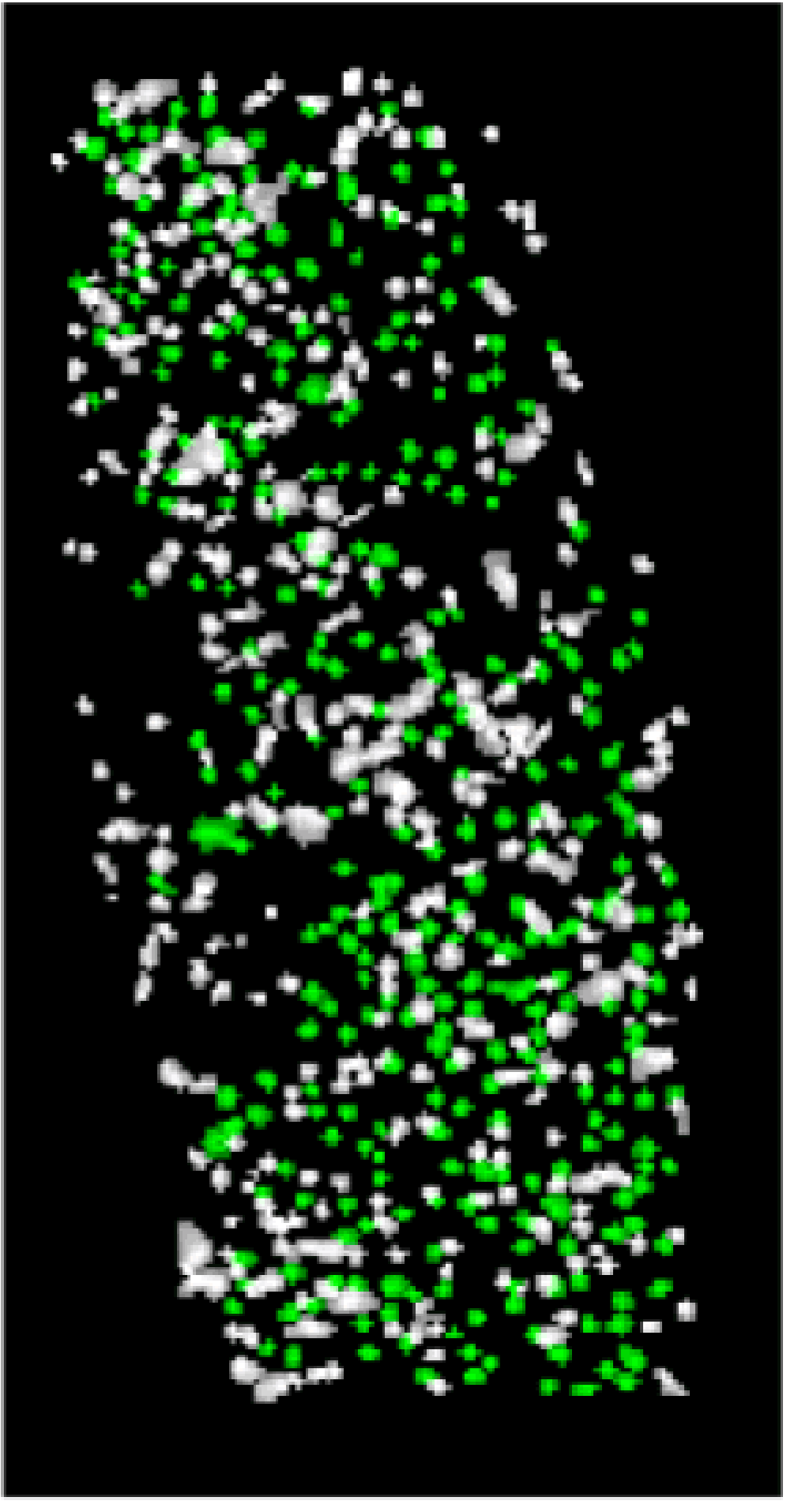

D

$\int_{11}^{15}$

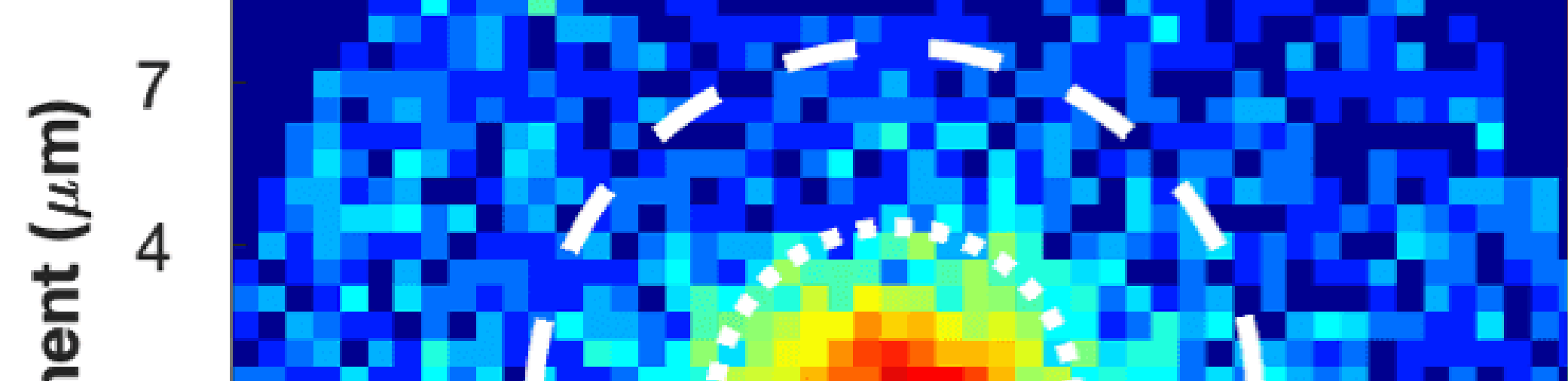

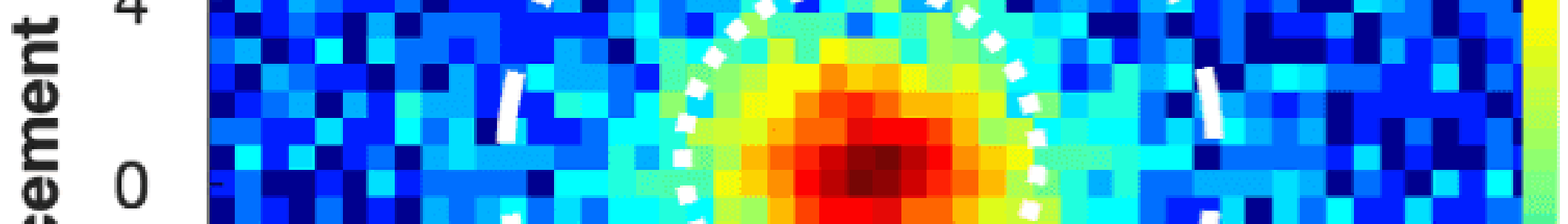

造 0

$\underbrace{\frac{\pi}{2}}_{-7}$

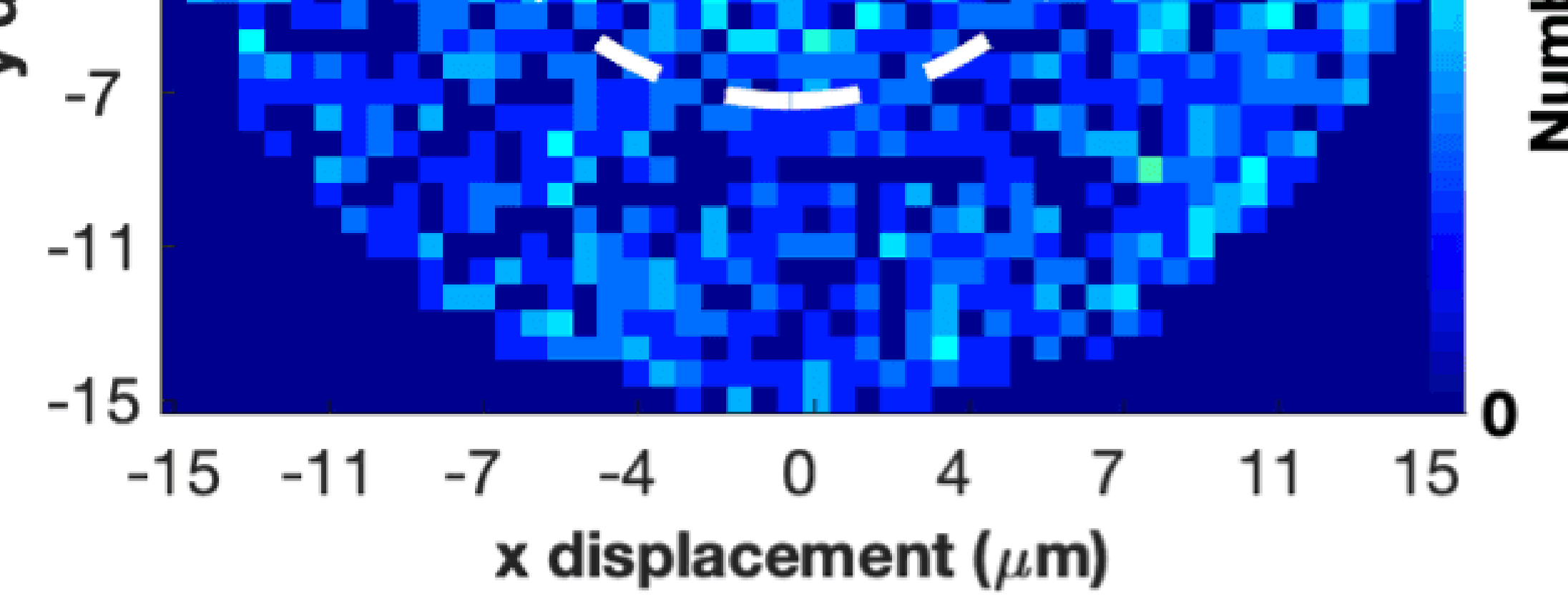

E

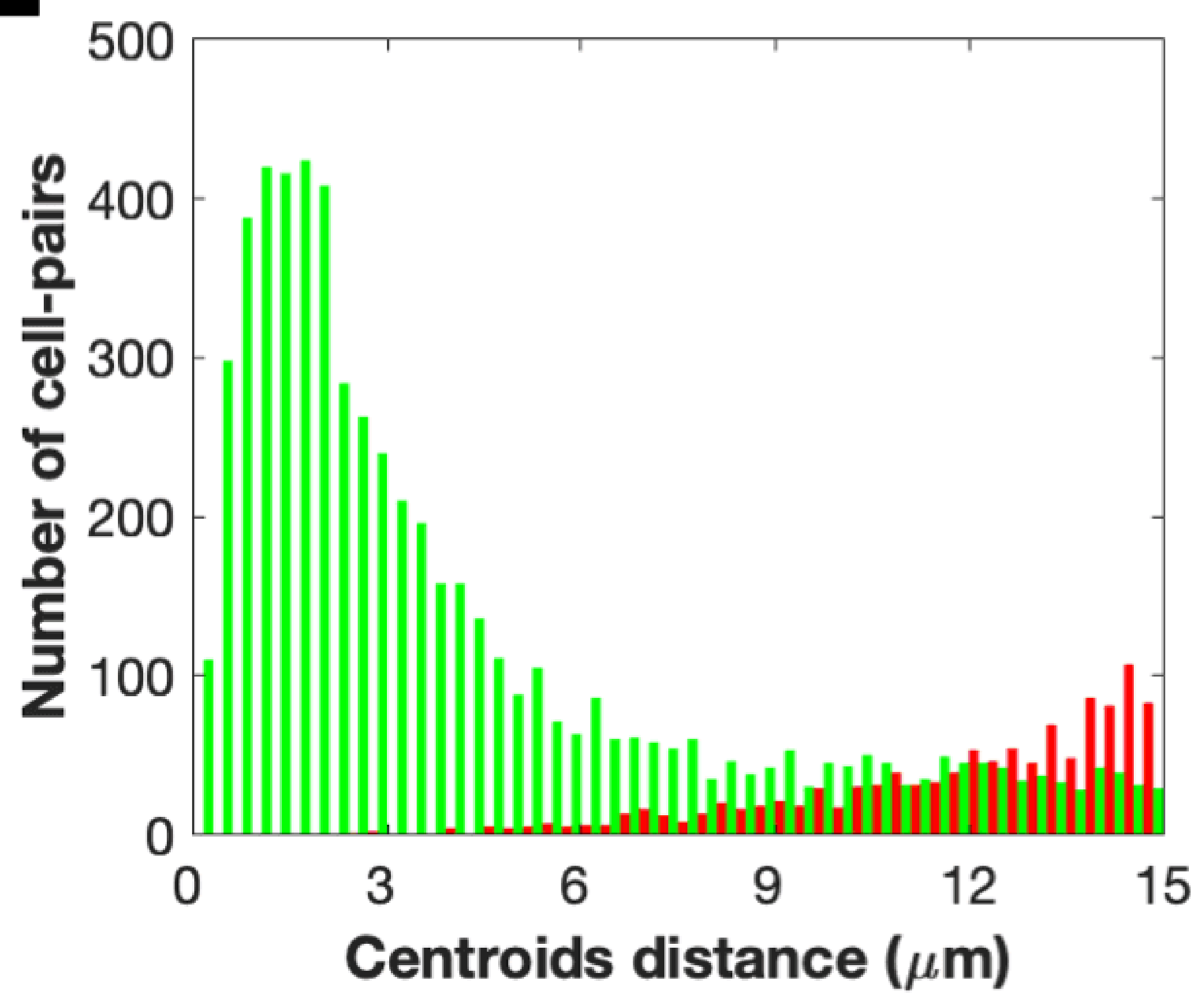




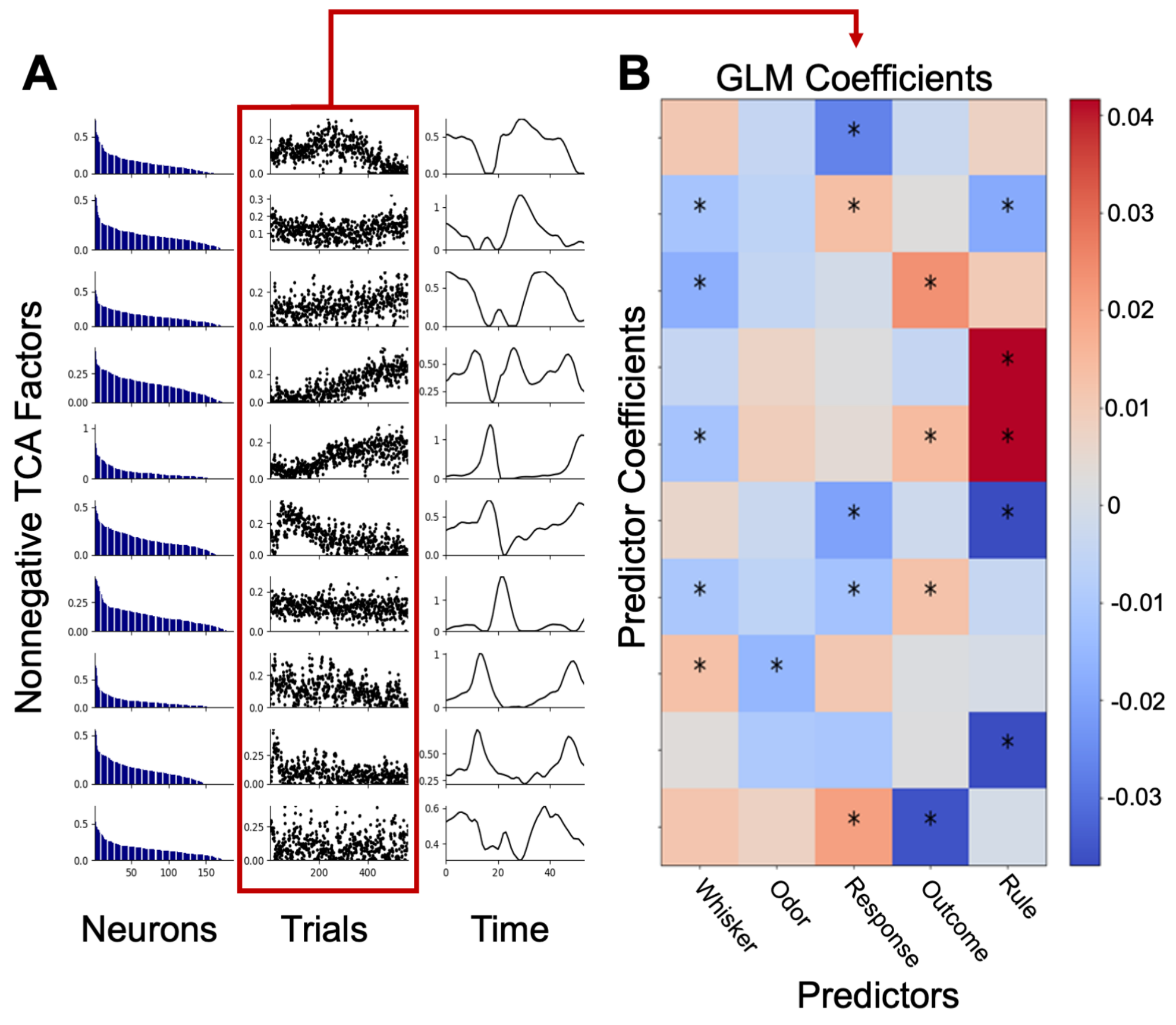


A response direction

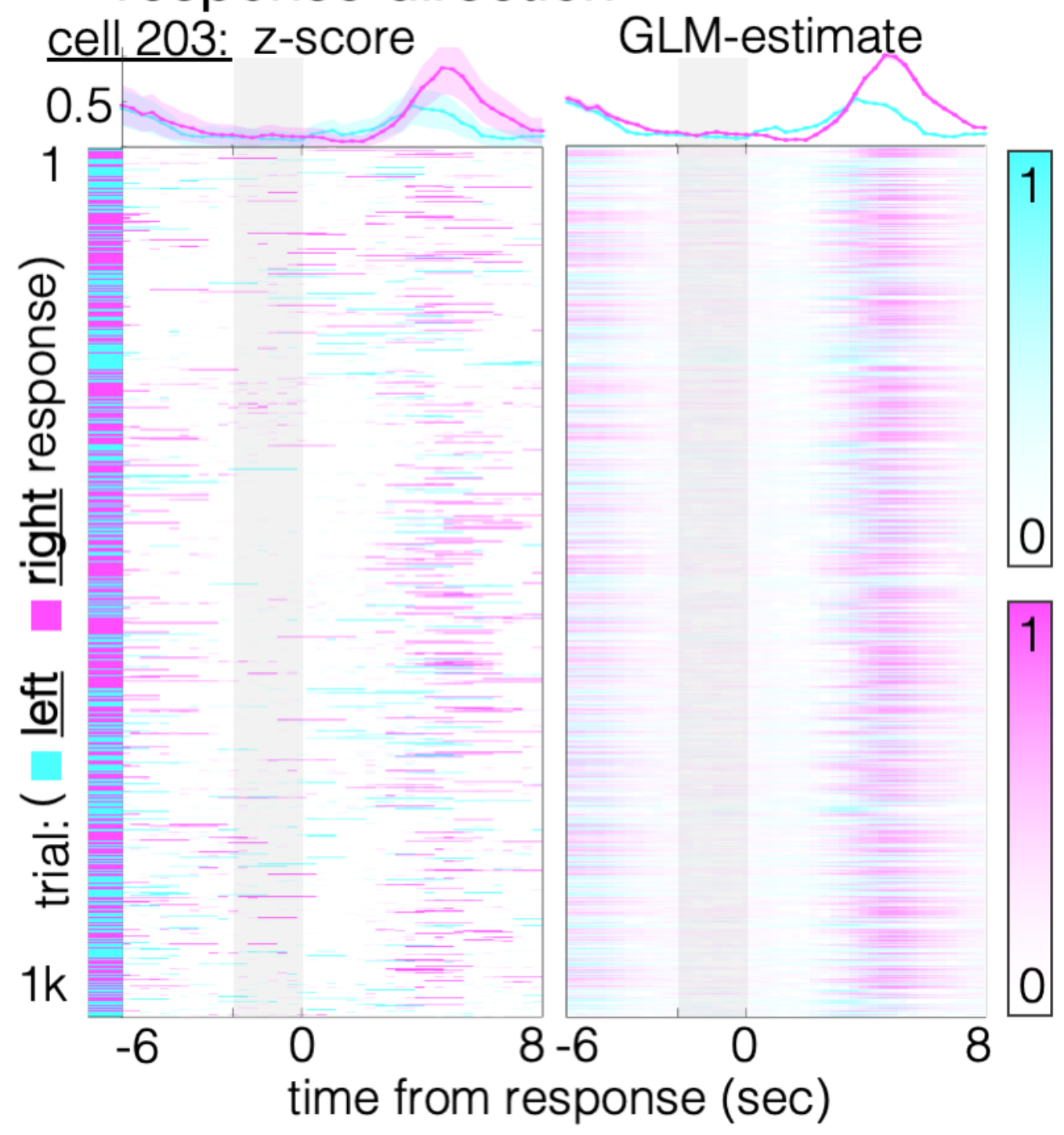

B outcome

cell 211: z-score GLM-estimate

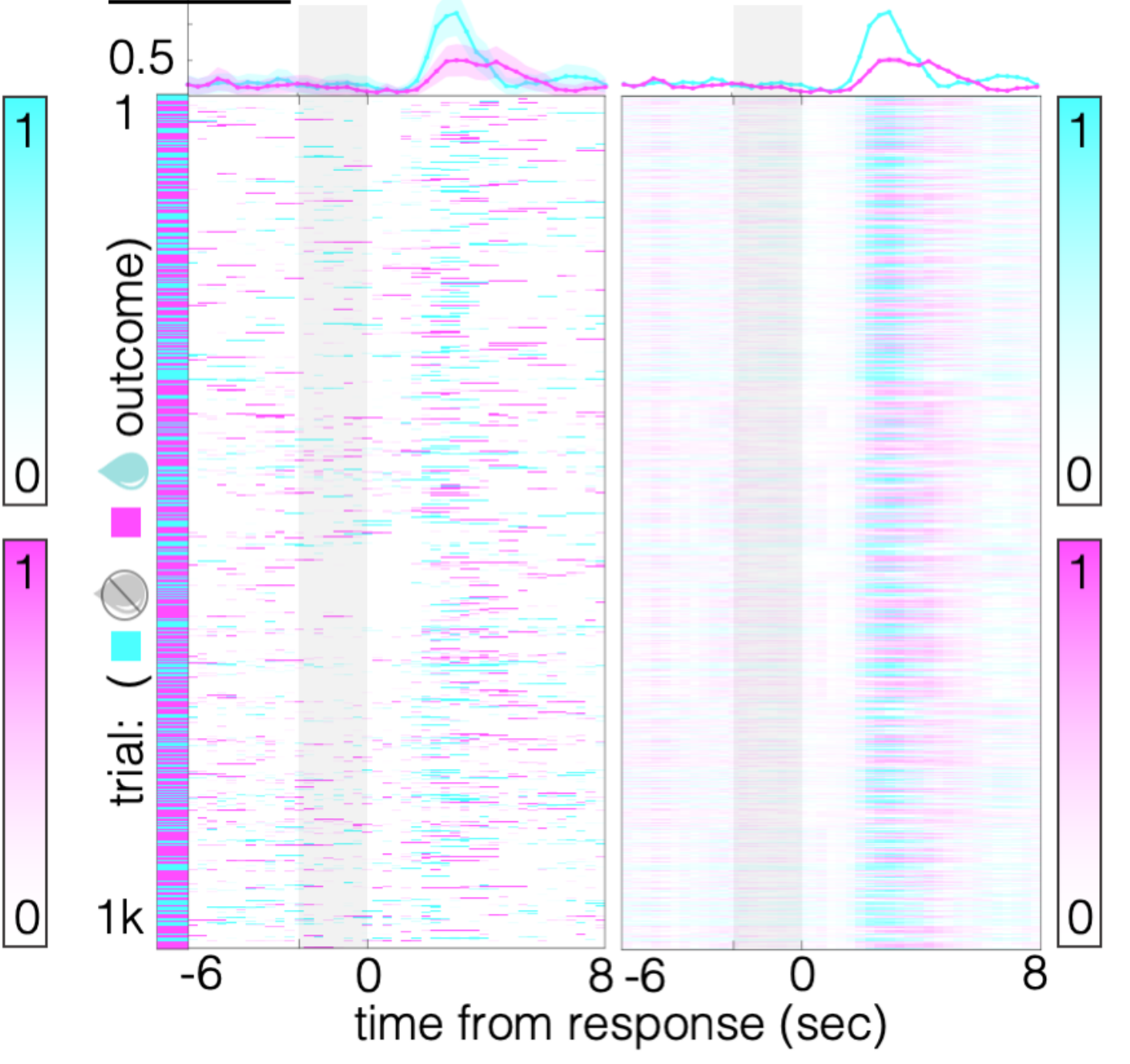



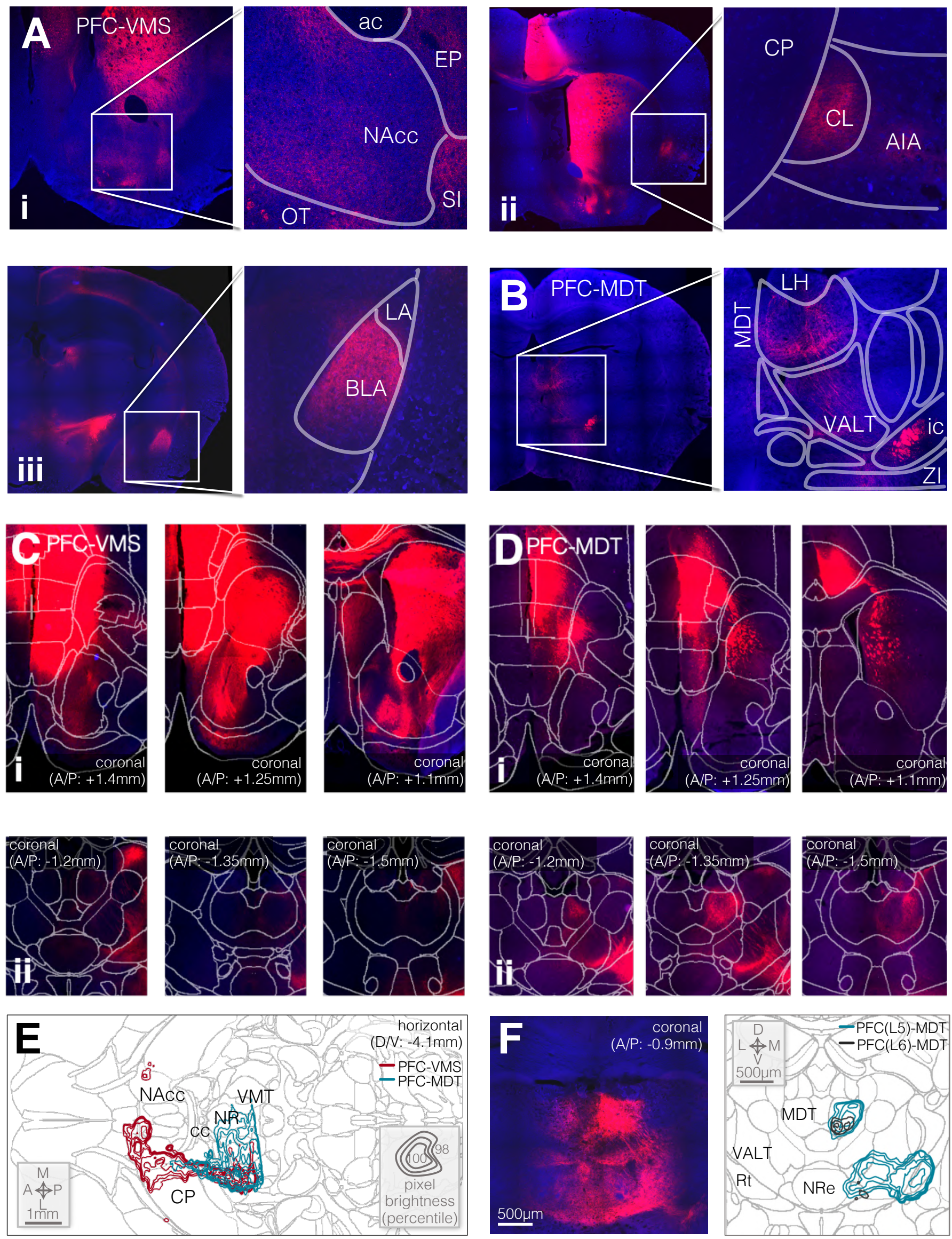

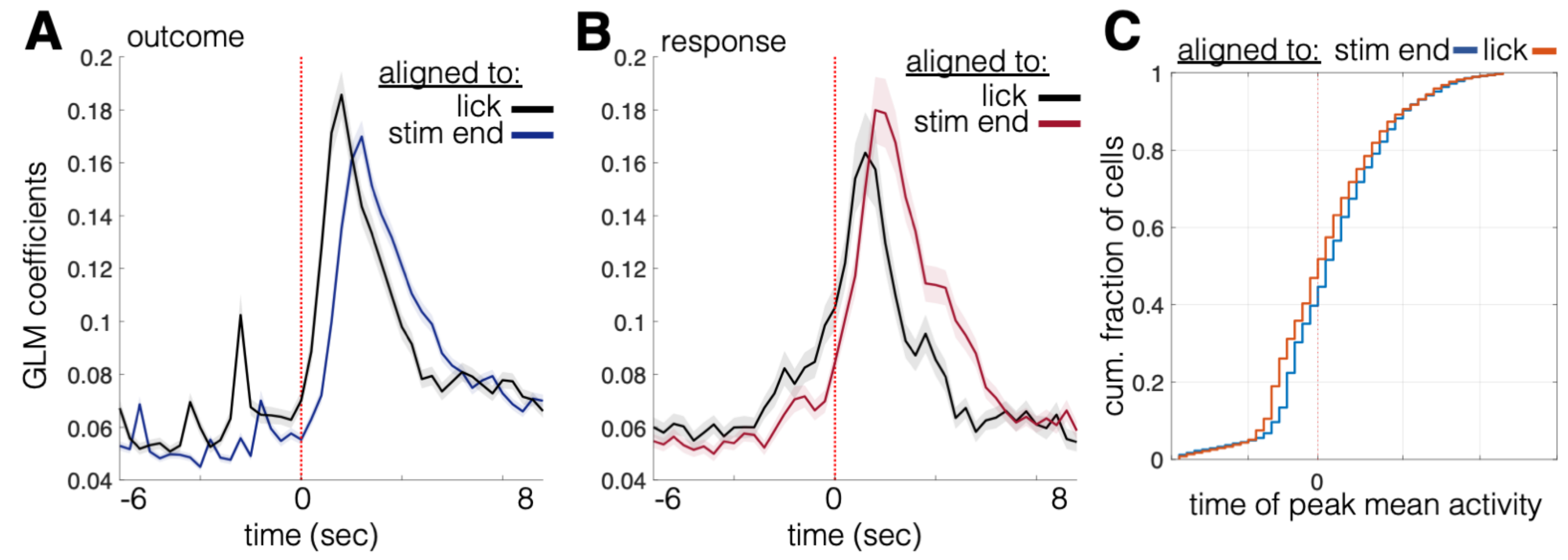